\title{
THE CORONAL X-SPECTRUM: PROBLEMS AND PROSPECTS*
}

\author{
A. B. C. WA LKER, JR. \\ Space Physics Laboratory, The Aerospace Corporation, El Segundo, Calif. 90245, U.S.A.
}

\section{Introduction}

The coronal spectrum provides a unique opportunity to probe atomic processes in a high temperature plasma. The corona is optically thin, at low density, and the coronal ions are highly stripped. These characteristics result in an emission line spectrum with a rich multiplet structure, with forbidden lines prominent in the spectrum, and deexcitation of metastable states by two photon decay making significant contributions to the continuum. The multiplet structure is further complicated by the presence of satellite lines from autoionizing states formed during recombination. With few exceptions, the true coronal lines are below $300 \AA$, and the general necessity for and complexity of grazing incidence optics has made it difficult to obtain high resolution spectroheliograms.

In principle, the physical state of the coronal plasma should be directly calculable from the characteristics of the spectrum since in the optically thin corona the source function for permitted lines is independent of the detailed structure of the emitting regions. The sensitivity of the relative population of metastable states in several isoelectronic sequences to electron density provides an additional diagnostic technique for understanding the structure and dynamics of the coronal plasma. However, the complex variation of the temperature and density of the corona across the disk have greatly complicated the interpretation of the available high resolution spectral observations, which have been of the whole disk. In a few cases, the domination of the spectrum at the shortest wavelengths by a few active regions and the one dimensional imaging characteristics of Bragg crystals have helped circumvent this problem. Grazing incidence imaging techniques, although as yet unable to resolve individual lines or multiplets, are invaluable in interpreting the coronal spectrum.

The coronal spectrum below $25 \AA$ has been studied using crystal spectrometers from rockets and satellites for a variety of levels of solar activity and virtually all lines observed from the non-flaring Sun have been identified. The spectrum is dominated by the lines of the hydrogen- and helium-like ions of the lighter abundant elements $(\mathrm{N}, \mathrm{O}$, $\mathrm{Ne}, \mathrm{Mg}, \mathrm{Si}, \mathrm{S}$ ) and of the neon-like and fluorine-like ions of iron. The identification of the line resulting from the forbidden single photon decay of the $2^{3} S$ state of heliumlike ions and the dependence of the population of this state on electron density has provided a tool which may prove most useful in understanding the structure of the

* This work was conducted under U.S. Air Force Space and Missile Systems Organizatisn (SAMSO) Contract No. F 04701-70-0059. 
corona. Spatially resolved spectra must be obtained before this technique can be effectively utilized. The observation of strong dielectronic recombination satellite lines near the helium-like multiplets should make the study of these multiplets with high resolution extremely productive.

Observations of the coronal spectrum between 18 and $400 \AA$ have been carried out by a number of groups using grazing incidence spectrographs on rockets and satellites. The spectrum in this range contains the lithium- and beryllium-like lines of several ions ( $\mathrm{Ne}, \mathrm{Mg}, \mathrm{Si}, \mathrm{S}$ ) but is dominated by lines from a broad range of ionization stages of iron and silicon. The identification of the iron lines, in particular, demonstrated the close interdependence between studies of the solar plasma and laboratory plasmas. The study of these lines has been most fruitful; since the broad temperature range, extending well down into the transition region, over which these iron and silicon lines are formed has allowed the construction of coronal models independent of the vexing abundance problem. There are, however, still many unidentified lines in this spectral region.

There are a number of metastable levels which give rise to multiplets which are of special interest in diagnostic studies of the coronal plasma. An example is the ground state of boron-like ions such as Six, which is a doublet, with the two levels weakly coupled by a forbidden transition. The relative population of these levels, and consequently the intensity distribution within multiplets ending in these levels, is density dependent for densities normally found in the coronal plasma.

The absolute and relative photometry of the solar coronal spectrum cannot be considered to be in a satisfactory state at this time, and a number of important problems, including the question of coronal abundance, cannot be satisfactorily resolved without improvements in the experimental observations. The study of the coronal spectrum with good spectral and spatial resolution is the next step in understanding the structure and dynamics of the corona. The use of grazing incidence optics and high resolution multigrid mechanical collimators should provide the experimental observations required over the next few years.

In this review I shall concentrate on high resolution observations of the spectrum of the non-flaring Sun. A number of recent reviews (Pounds, 1970; Neupert, 1970; Neupert, 1969; Underwood, 1968; Goldberg, 1967; Mandel'stam, 1967) have discussed the results of a broader range of observational techniques for all levels of solar activity.

\section{The Observational Status}

The coronal spectrum breaks into two distinct regions near $25 \AA$, for reasons related both to instrumental techniques and to the structure of the corona. The spectrum below $25 \AA$ contains lines which are efficiently excited at temperatures of $1.5 \times 10^{6}$ $2 \times 10^{6} \mathrm{~K}$ or higher and are, therefore, true coronal lines. The spectrum in this wavelength region has been investigated primarily by crystal spectroscopy. Between 25 and $400 \AA$ many of the lines which dominate the spectrum have substantial excitation efficiency at temperatures below $1.5 \times 10^{6} \mathrm{~K}$ and are produced partly in the transition 
region and partly in the corona. The observational technique which has been used almost exclusively in this wavelength region is the grazing incidence grating.

\subsection{The SPECTRUM BELOW $25 \AA$}

Since the observations of the first high resolution X-ray spectra of the Sun by the NRL group (Blake et al., 1964, 1965) and by the group at the Lebedev Institute
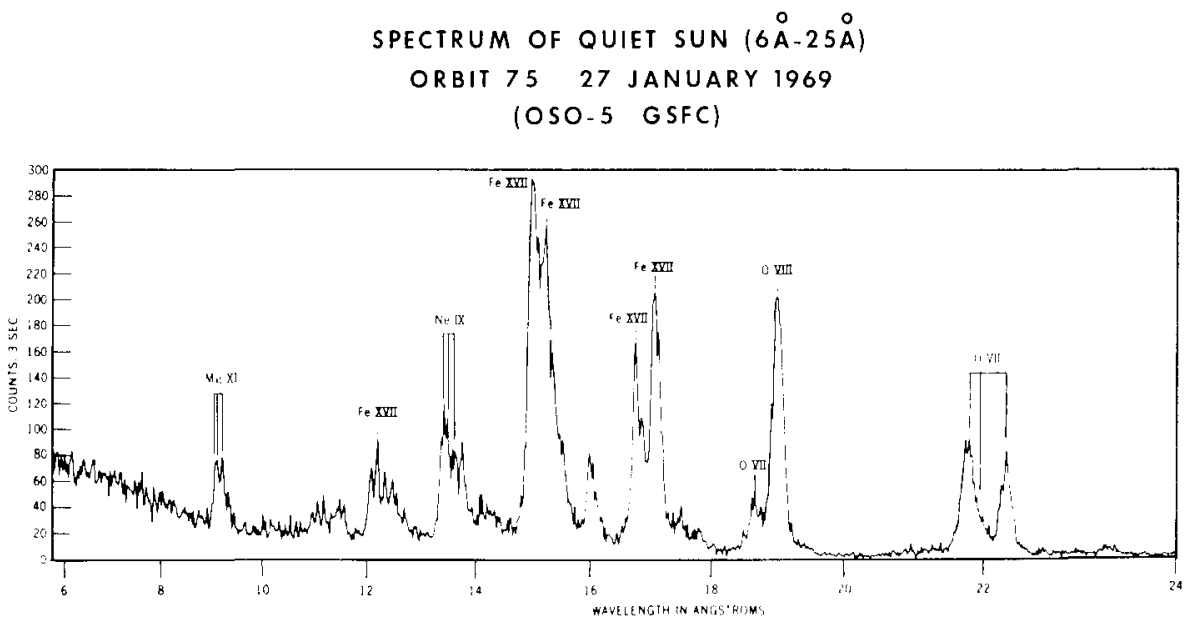

Fig. 1. Solar spectrum obtained on Jan. 27, 1969 by OSO 5. (Courtesy of W. N. Neupert, GSFC) No-flare activity occurred near this time.

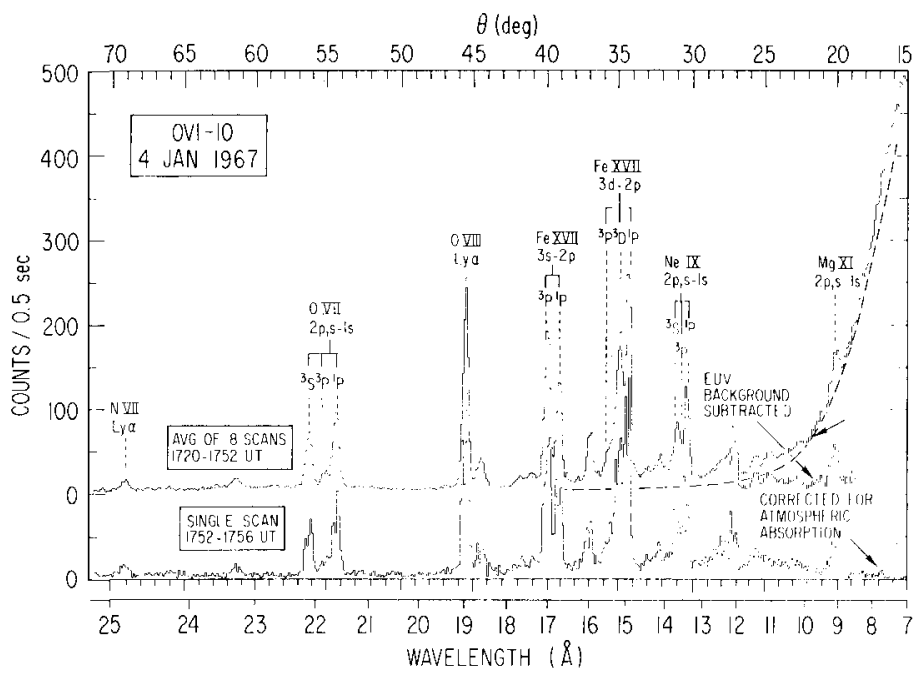

Fig. 2. Solar spectrum obtained on January 4, 1967 by OV1-10. No flare activity occurred near this time. The upper spectrum is the average of eight scans during which the spectrum was unchanged. The lower spectrum was obtained as the Earth's atmosphere occulted the Sun, attenuating the EUV scattered light background but not appreciably attenuating the $\mathrm{X}$-ray flux. 
(Zhitnik et al., 1965, 1967), the soft X-ray spectrum of the Sun has been observed with numerous rocket and satellite borne spectrometers.

In Table I we have expanded Pounds (1970) list of OSO soft X-ray experiments to include high resolution solar X-ray observations made on rockets and satellites for the spectral region below $25 \AA$. Many of the published satellite results have emphasized flare spectra, and we shall, in some cases, be forced to study flare spectra to discuss many of the weaker features in the spectra, which are difficult to distinguish clearly in quiet solar spectra due to limitations of instrument sensitivity. High resolution spectra have been reported by groups at the Naval Research Laboratory, at Goddard Space Flight Center, at The Aerospace Corporation, by a combined Leicester University and University College London group, and by the groups at Culham and at Lebedev Institute who have extended grazing incidence techniques down to $9 \AA$. References to published results are given in Table $\mathrm{I}$.

Figures 1 (Neupert, 1970) and 2 (Walker and Rugge, 1971a) show solar spectra obtained with KAP crystals on the OSO-5 and OVI-10 satellites. The spectra are quite similar and are typical of the quiet Sun. The spectra shown in Figure 2 illustrate several of the problems associated with available spectra obtained with satellite-borne spectrometers, scattered EUV backgrounds which make continuum observations difficult at the shorter wavelengths, and the importance of active regions which result in multiple lines for each emission feature. The upper spectrum is the average of eight scans and the multiple lines, caused by active regions approximately $18^{\prime}$ apart in the direction of scan, which are obvious in the single scan, are averaged out of the data. The single scan obtained between 1752-1756 UT is particularily interesting because it was taken as the satellite moved behind the Earth, causing the Earth's upper atmo-

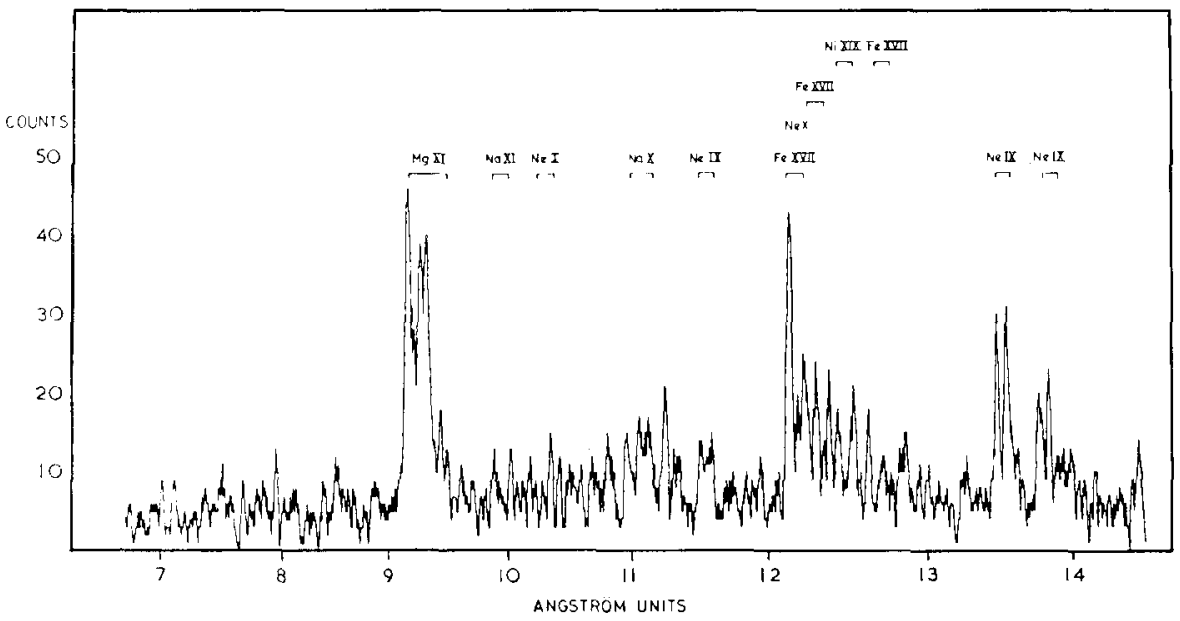

Fig. 3. Solar spectrum obtained on August 8, 1967 from a Skylark rocket. (Courtesy of K. A. Pounds). A beryl crystal was used, with a flow proportional counter detector so that scattered light was held to a minimum. There were three active regions on the disk, resulting in considerable overlapping of the observed spectral lines. 


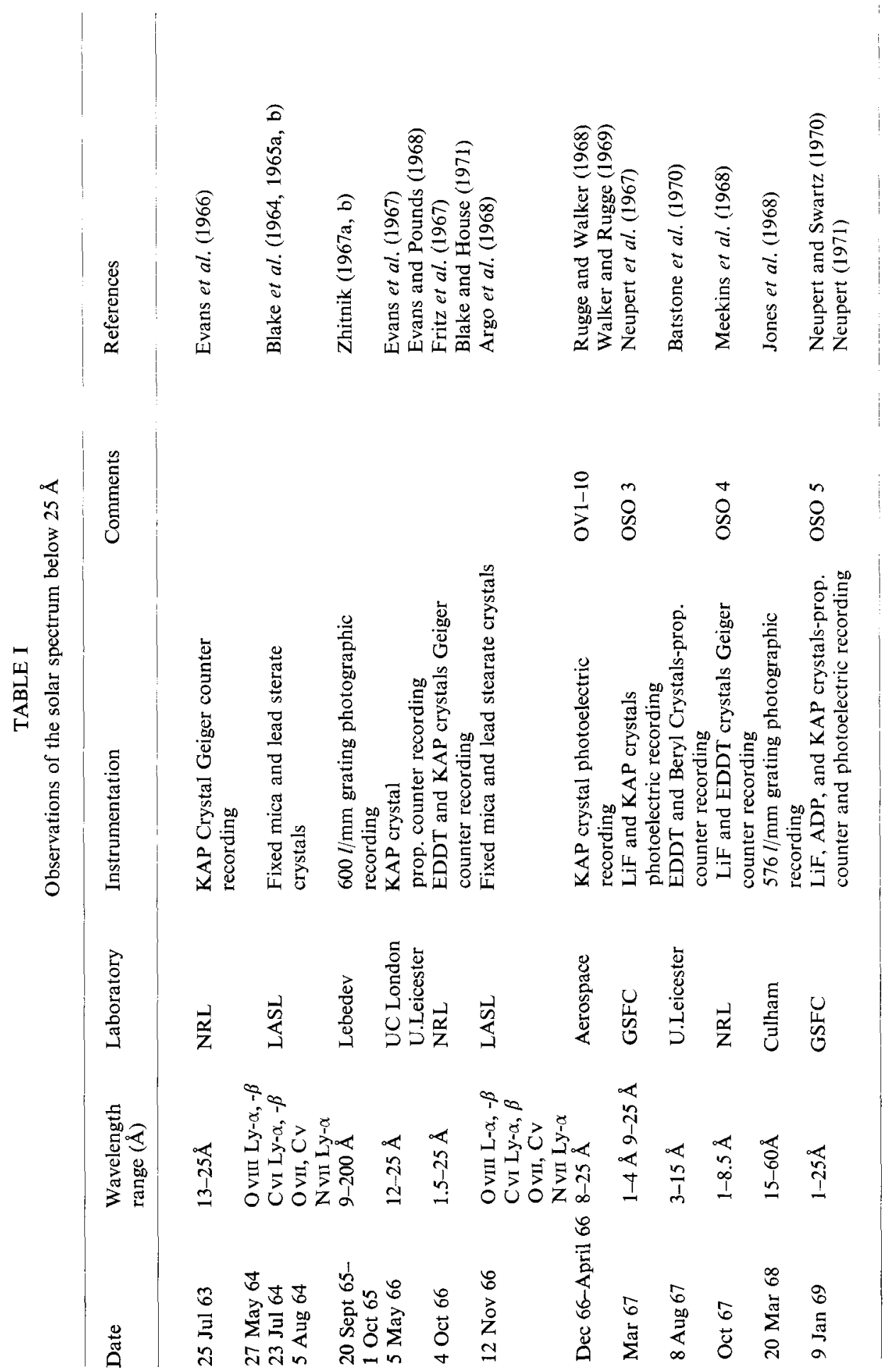




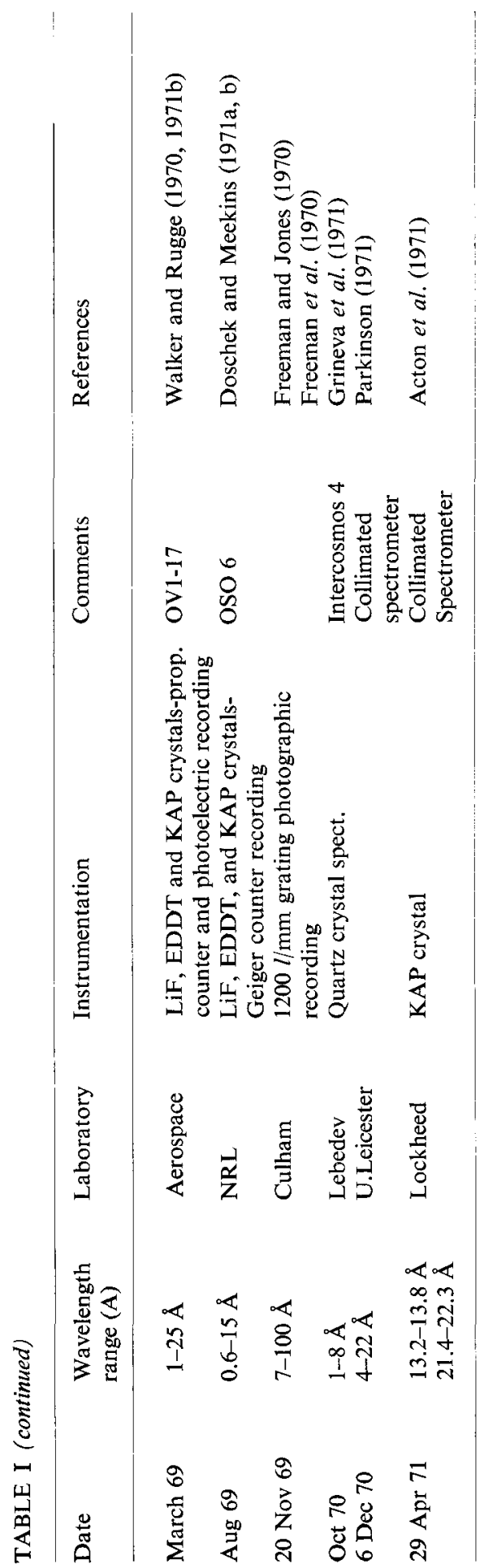


sphere to occult the Sun and attenuate the solar EUV flux (near $170 \AA$ ) which is the cause of the scattered light background, thereby allowing an accurate measurement of the X-ray continuum near $9 \AA$. For the quiet Sun, the intensity in the X-ray continuum is quite low compared to that in the lines, as both spectra show. Figures 3 and 4 illustrate the effect on the spectrum of several simultaneous active regions on the disk. These observations were obtained with a rocket-borne beryl crystal spectrometer by

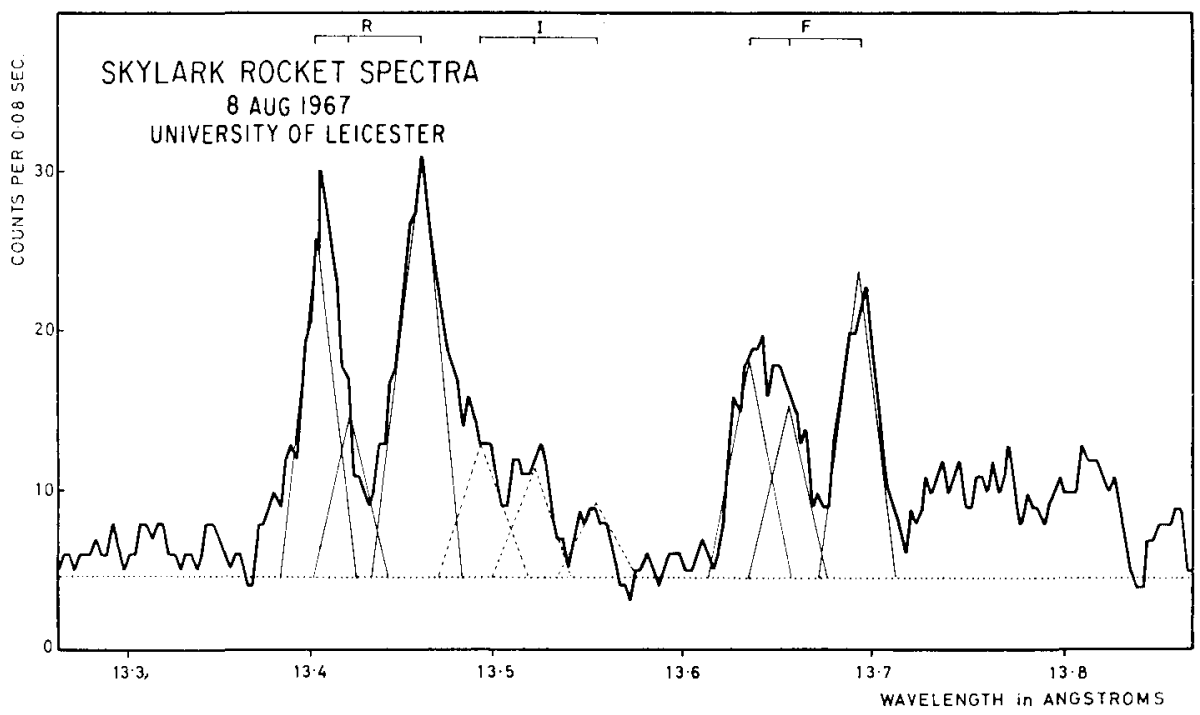

Fig. 4. Enlargement of the August 8, 1967 Skylark spectra, showing the decomposition of the Ne IX helium-like resonance, intercombination, and forbidden line into the contributions from each of the three active regions which were present on the disk.

the Leicester group (Batstone et al., 1970) and have higher resolution than the satellite observations. Figure 4 shows the contribution to the Ne Ix lines from three active regions on the disk. The active regions dominate the solar emission for the hotter lines such as NeIX $1 s^{2}{ }^{1} S-1 s 2 p^{1} P$, which is produced at temperatures of $3.5 \times 10^{6} \mathrm{~K}$ or higher.

All of the strong emission lines observed in non-flare spectra have been identified. Line lists for the spectral region between 8 and $25 \AA$ have been compiled by Fritz et al. (1967), by Evans et al. (1967), by Evans and Pounds (1968) and by Rugge and Walker (1968). The most significant departure from the earlier lists is the correct identification of the forbidden line, $1 s^{2}{ }^{1} S-1 s 2 s^{3} S$, in the multiplet structure of the lines from the helium-like ions. This identification was made by Gabriel and Jordan (1969a) after the high resolution grazing incidence grating spectra of Jones et al. (1968) demonstrated that the single strong satellite to the $\mathrm{O}$ VII $1 s^{2}{ }^{1} S-1 s 2 p^{1} P$ and ${ }^{3} P$ lines did not coincide in wavelength with the satellite lines observed in the laboratory, but rather matched the 
energy of the $1 s^{2}{ }^{1} S-1 s 2 s^{3} S$ transition.* The laboratory satellites, which are due to doubly excited configurations of the lithium-like ion, have subsequently been shown to be important for heavier ions in the corona (Walker and Rugge, 1971b). We shall discuss the problem of line identification more fully in a subsequent section. Figure 5 shows the spectra of the Sun for a more active period, and allows the observation of a large number of lines which are difficult to observe in the spectra of the quiet Sun. When one region dominates the solar X-ray emission, as is the case in Figure 5, the

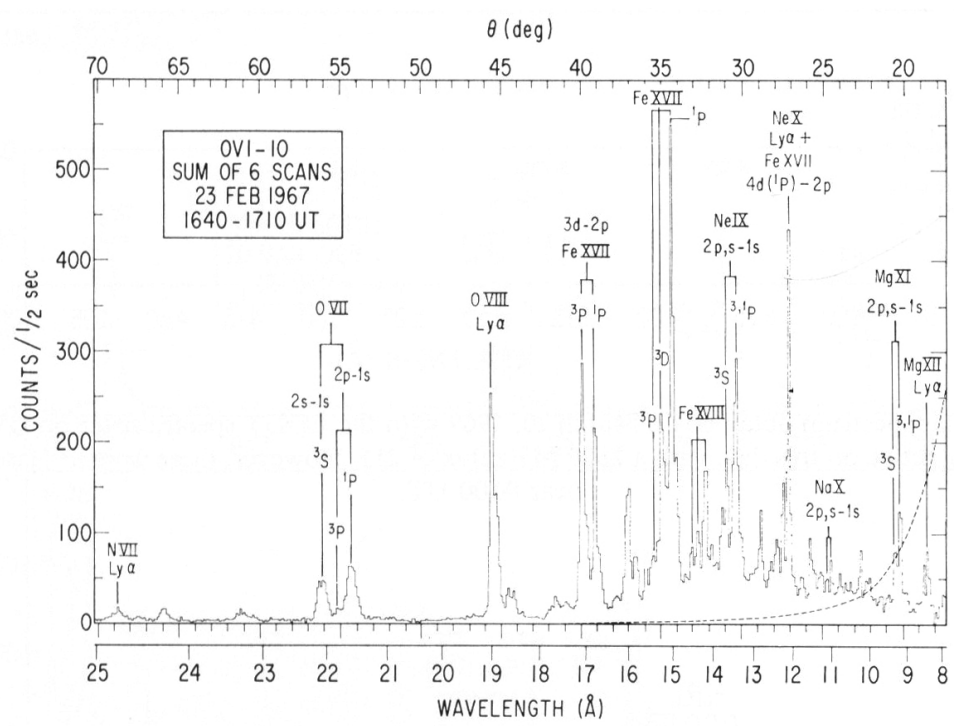

Fig. 5. Solar spectrum obtained shortly after a class-1 B flare on February 23, 1967 by the OV1-10 satellite. The enhanced flux in this spectrum, and the domination of the spectrum at short wavelength by a single coronal region, greatly facilitates line identification.

effective resolution of slitless crystal spectrometers is enhanced, and this fact together with the increased statistics greatly simplifies the problems of line identification.

The spectral region below $8.5 \AA$ is highly variable due to its domination by lines formed, with a few exceptions, at temperatures of $6 \times 10^{6} \mathrm{~K}$ or higher. The NRL group published the first observation of the spectrum in this range (Fritz et al., 1967). Figure 6a shows a more recent observation made with an EDDT spectrometer on the OVI-17 satellite (Walker and Rugge, 1970). Figure 6b (Doschek and Meekins, 1970) shows the sum of 50 'low intensity scans' (lower curves) which correspond to periods of high solar activity, including small $(-\mathrm{F},-\mathrm{N}$, or $1 \mathrm{~N})$ flares. The upper curve Figure $6 \mathrm{~b}$ shows the sum of 50 spectra associated with larger (1B) flares. During a period of $90 \mathrm{~min}$, which includes the time period of the scan shown in Figure 6a, there

* Rugge (1969) has extrapolated the wavelength of this line from data given by Moore (1949). His results are $\mathrm{Cv}, 41.472 ; \mathrm{N}$ VI, 29.534; O viI, 22.098; NeIX, 13.701; MgXI, 9.314; AlxII, 7.871; Si XIII, 6.739; Sxv, 5.100; and AxvII, 3.995. (All wavelengths are in $\AA$.) 


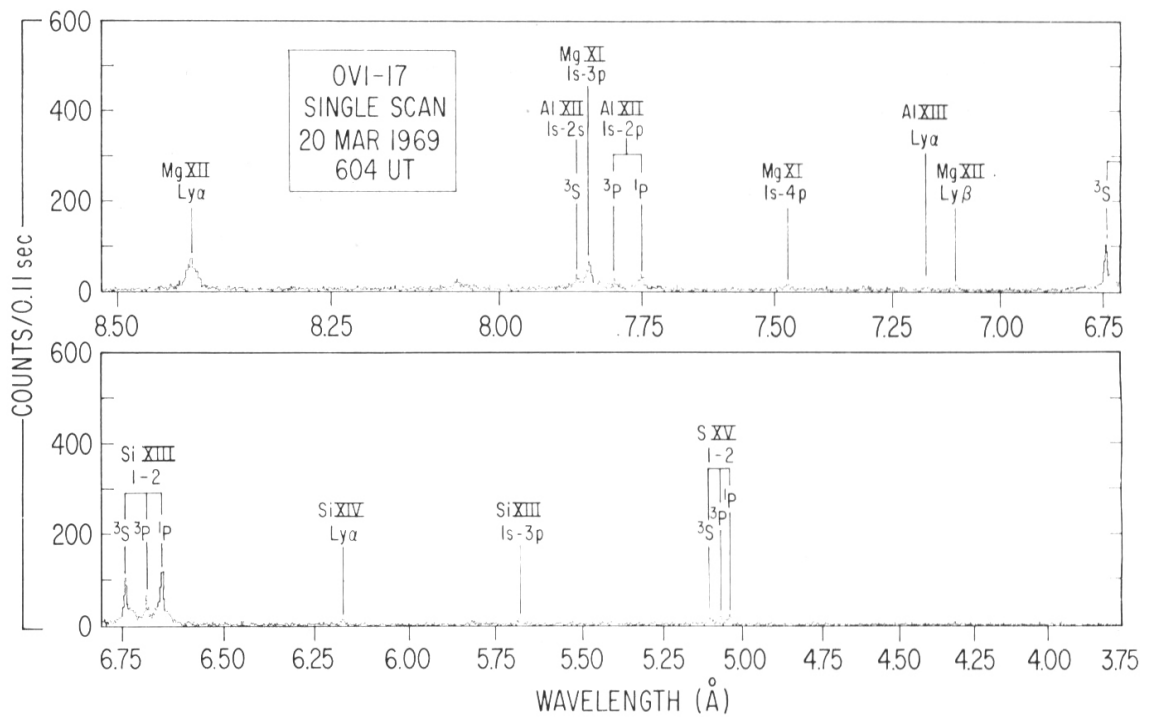

Fig. 6a. Solar spectrum obtained on March 20, 1969 with the EDDT spectrometer on OV1-17. The Sun was very active on this day, with a $2800 \mathrm{MHz}$ flux of 215 . However, there were no flares reported near $0600 \mathrm{UT}$.

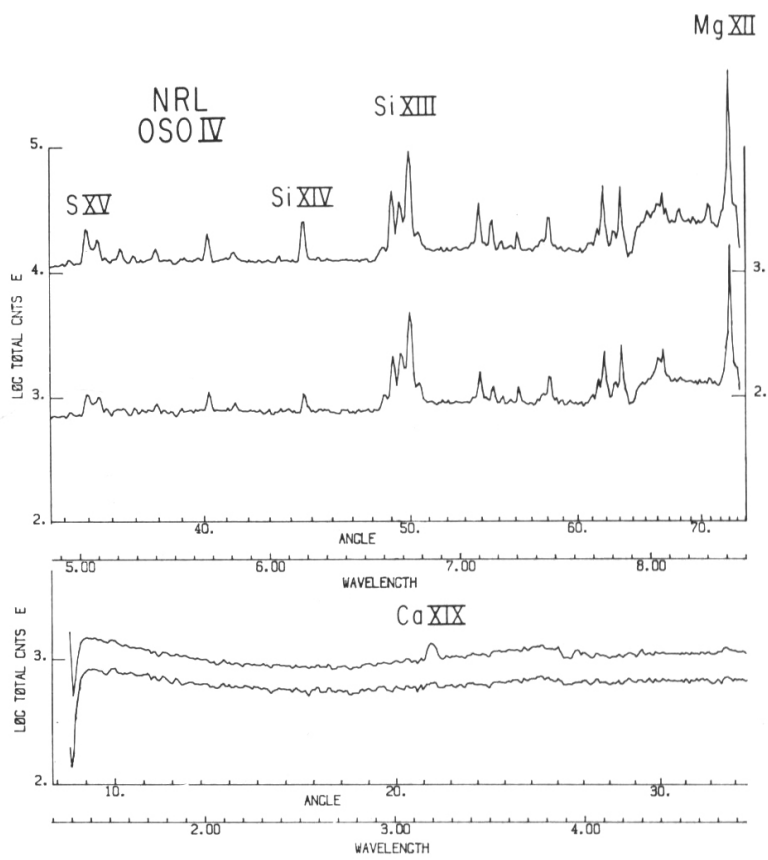

Fig. 6b. Average of 50 spectral scans obtained on OSO 4. The lower curve is composed from scans obtained in the absence of large flares (Courtesy of G. Doschek). 
was no change in the soft $\mathrm{X}$-ray flux. This observation demonstrates that active regions can contain stable cores with temperatures high enough to excite weak SxV and SixIV emission requiring temperatures near $8-10 \times 10^{6} \mathrm{~K}$. Figure 7 is a spectrum obtained approximately 40 min after a $-\mathrm{B}$ flare, and the enhanced flux in the spectra simplifies the problem of line identification. Fritz et al. (1967), and Walker and Rugge (1970) have given line lists for the spectral region below $8.5 \AA$. The results of Fritz et al., if correctly interpreted, are particularly interesting because of the observation of Fexxv, Caxx, and ArxvIII lines which imply very high temperatures (see also Blake and House, 1971).

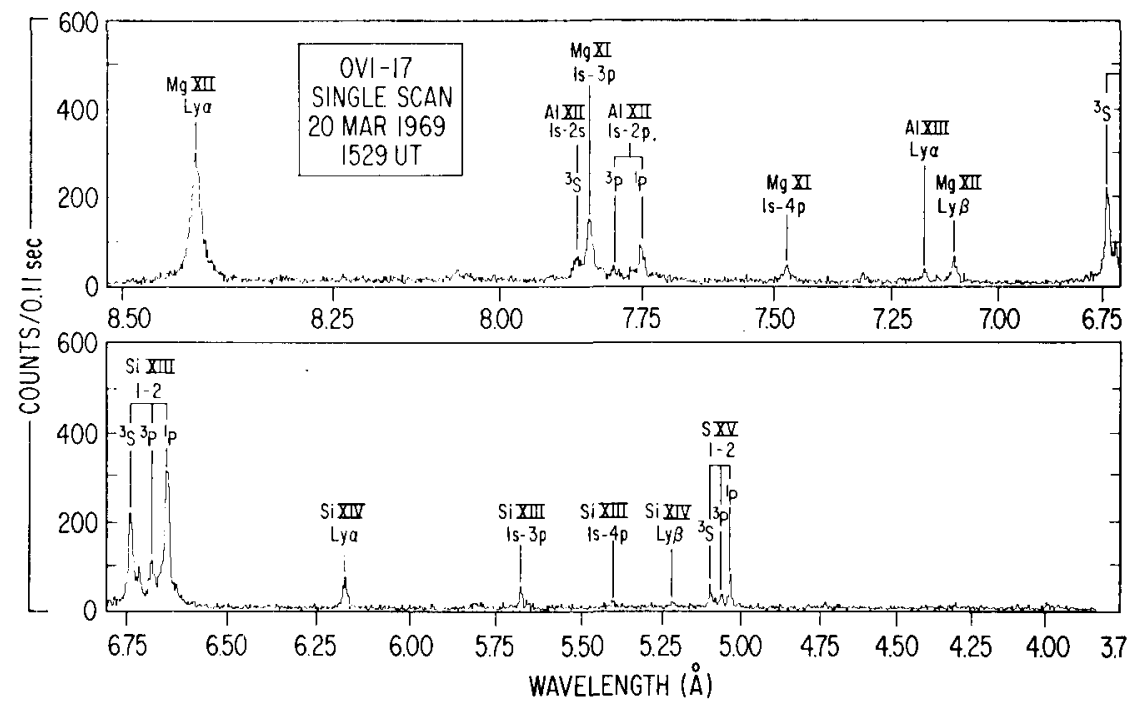

Fig. 7. Solar spectrum obtained at 1529 UT on March 20, 1969. A class-B flare was reported at $1450 \mathrm{UT}$.

Meekins et al. $(1968,1970)$ and Neupert (1971), have observed the solar spectrum during large flares, and have also compiled line lists based on the observation of several events. There appear to be some differences in flare and non-flare spectra which involve inner shell excitation of heavier ions, possibly in a non-equilibrium plasma. Blake and House (1971) discuss this point in some detail. There does not, however, appear to be evidence for nonequilibrium conditions in quiet Sun spectra.

The X-ray continuum between 10 and $25 \AA$ has been discussed by Evans and Pounds (1968) and Walker and Rugge (1969). The continuum below $10 \AA$ has been observed by Walker and Rugge (1970) for quiet conditions, and by Meekins et al. $(1968,1970)$ and Neupert and Swartz (1970) during flares. As we shall point out later, the spectral shape and intensity in the continuum can be of considerable interest in interpreting the structure of the coronal plasma. 


\subsection{The SPECTRUM From $25 \AA-400 \AA$}

Tousey et al. (1965) and Hinteregger (1965) have summarized the early rocket measurements of the solar spectrum in this wavelength range. A series of grazing incidence grating spectrographs, by the NRL group using photographic recording, and by the AFCRL group using photoelectric recording techniques mapped the solar spectrum down to $33.7 \AA$, the wavelength of the Lyman- $\alpha$ line of C VI. The GSFC group, using photoelectric recording techniques, has made observations between $170 \AA$ and $370 \AA$ on the OSO 1 satellite (Neupert et al., 1964), and between $120 \AA$ and $400 \AA$ in a rocket flight of a prototype of the OSO instrument (Neupert and Behring, 1962). A number of authors (Tousey et al., 1965; Jordan, 1965; Ivanov-Kholodny and Nikolsky, 1963; Zirin et al., 1963 and Zirin, 1964) made line identifications based on these early spectra, however a number of lines remained unidentified. The most serious difficulties were presented by a group of intense lines between 170 and $220 \AA$. Careful comparison of the spectrum produced by iron-doped theta pinches (especially the Zeta discharge at Harwell) with the solar spectrum demonstrated that these lines were due to $3 p-3 d$ transitions in various stages of ionization of iron (Gabriel et al., 1966; Fawcett et al., 1967). The excellent review of the solar XUV and EUV spectrum by Goldberg (1967) summarizes the extensive comparison of laboratory and solar spectra by several groups which led to the identification of these lines.

More recent observations by the NRL, AFCRL, Culham and Lebedev Institute groups from rockets, and by the AFCRL and GSFC groups on the OSO satellites have provided calibrated solar XUV spectra which have allowed detailed models of the transition region and corona to be constructed. In Table II, we have listed grazing incidence rocket and satellite measurements and given references to published results. This is an extension of the table compiled by Goldberg (1967). We have not included
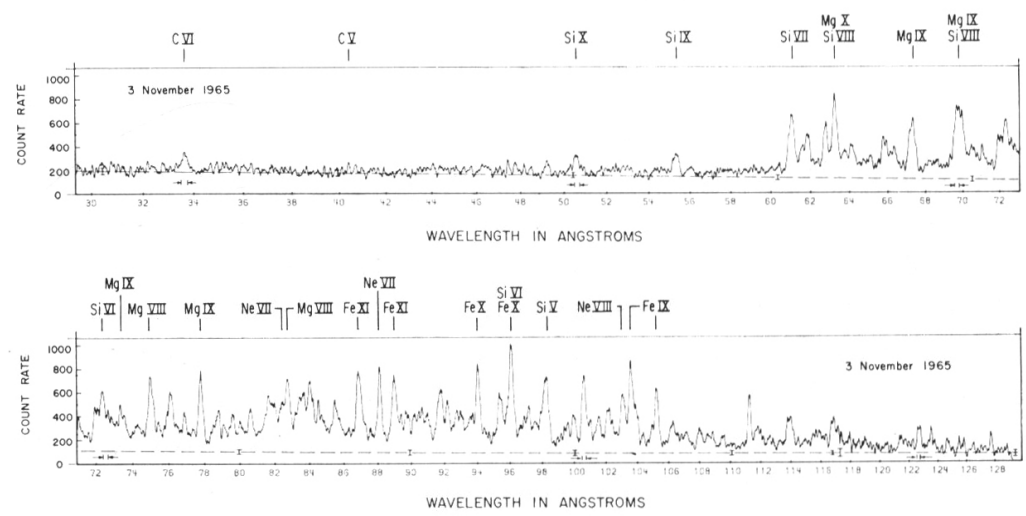

Fig. 8. Non-flare solar XUV photoelectric spectrum for November 3, 1965. The spectrum has been improved in resolution by the use of improved data reduction techniques. The horizontal arrows give the fwhm resolution, and the vertical bars the per sample statistical fluctuations of the background level (Courtesy of J. Manson). 

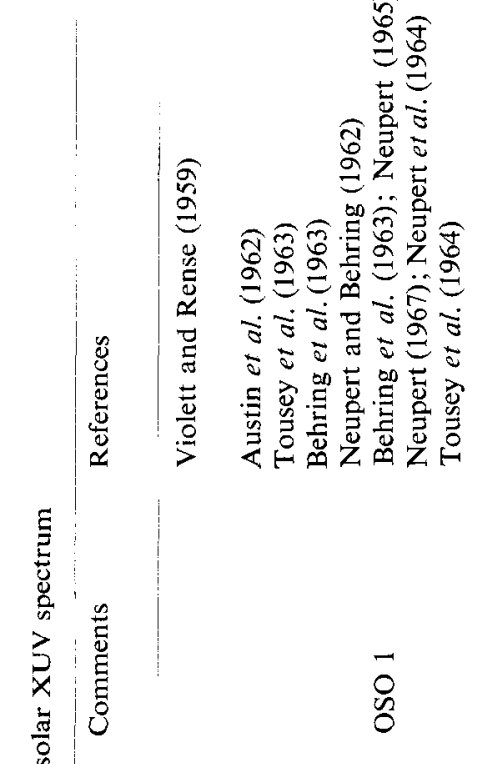

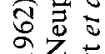

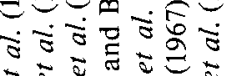

$\ddot{6}$

竎

$+\stackrel{2}{=}$

สํํ를

要范

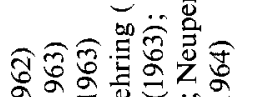

。气き

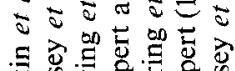

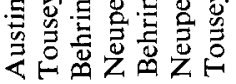

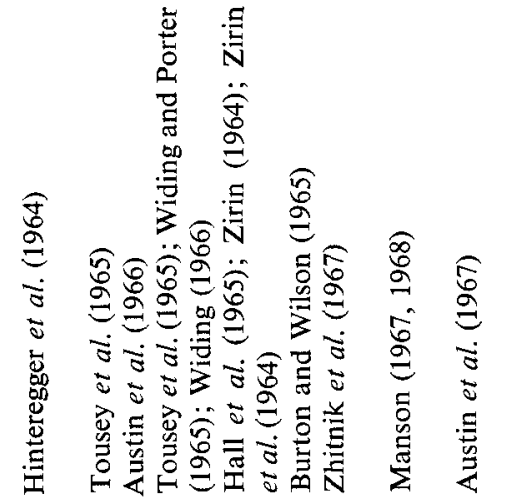

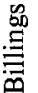

䒕

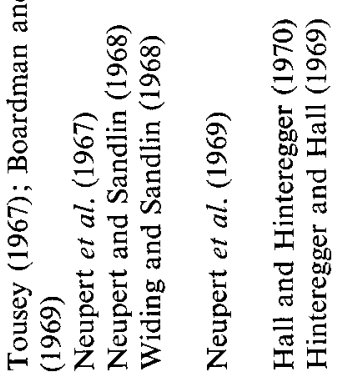

善

$\stackrel{\infty}{\stackrel{\infty}{\Xi}}$

$\begin{array}{ll}m & m \\ 0 & 0 \\ 0 & 0\end{array}$

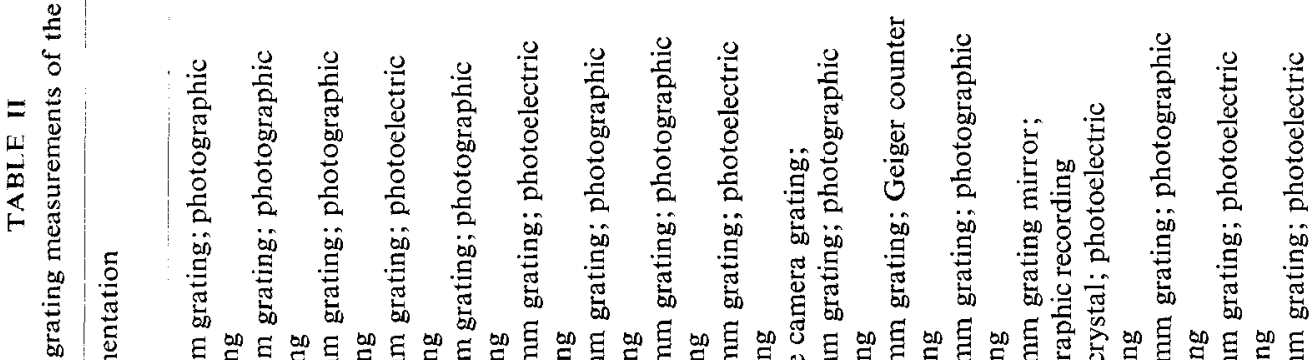

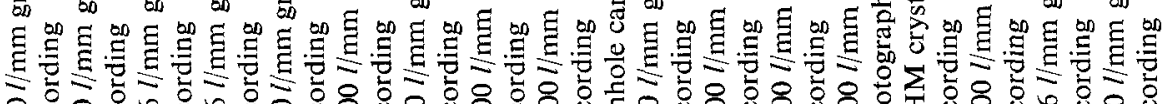

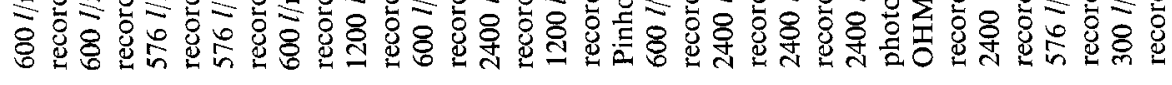

离

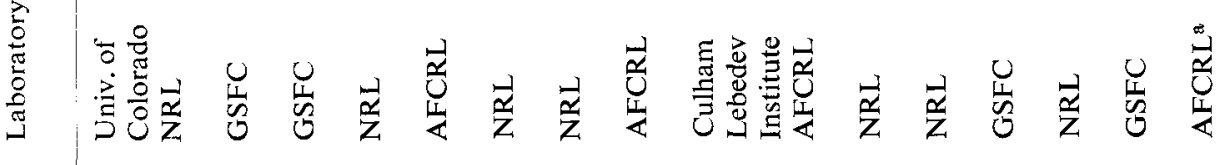

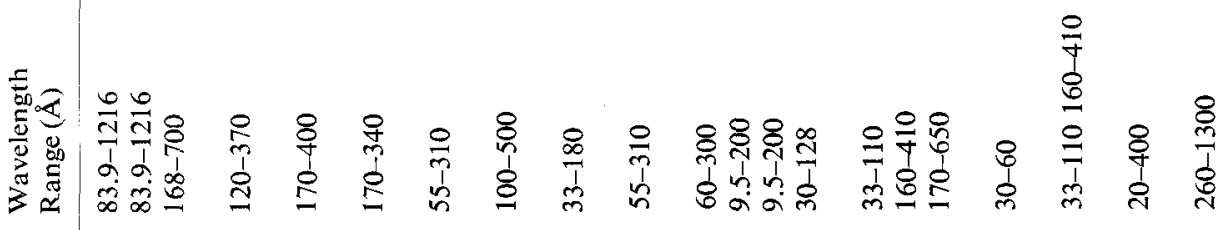
ชิ

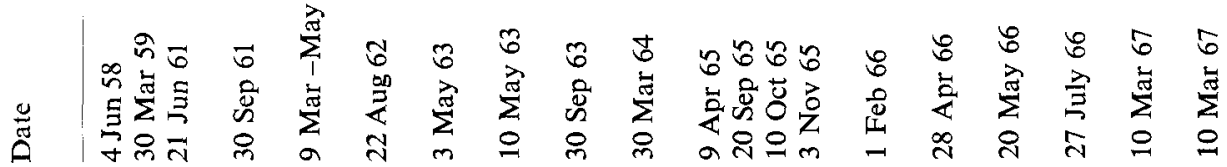


A. B. C. WALKER, JR.

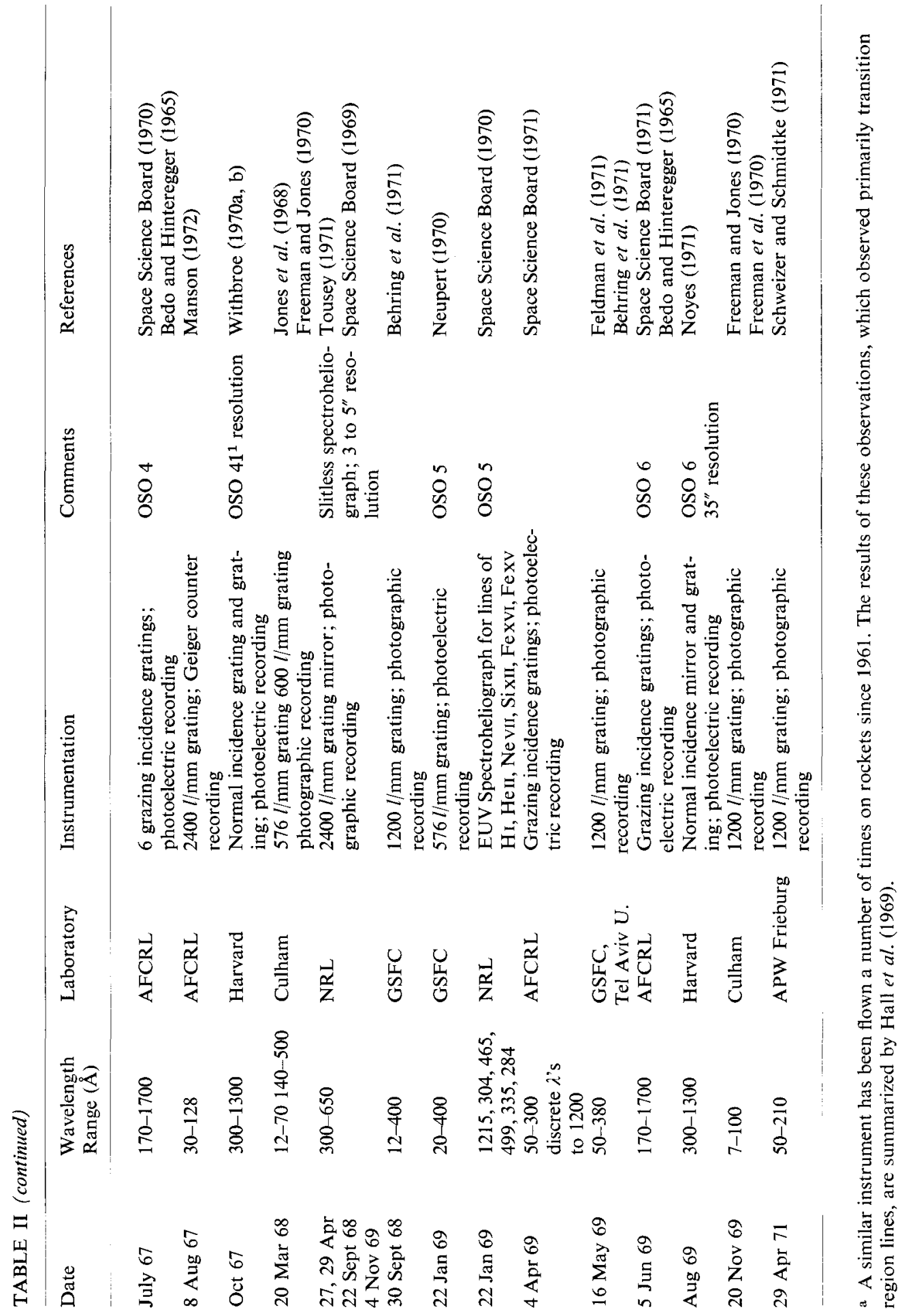




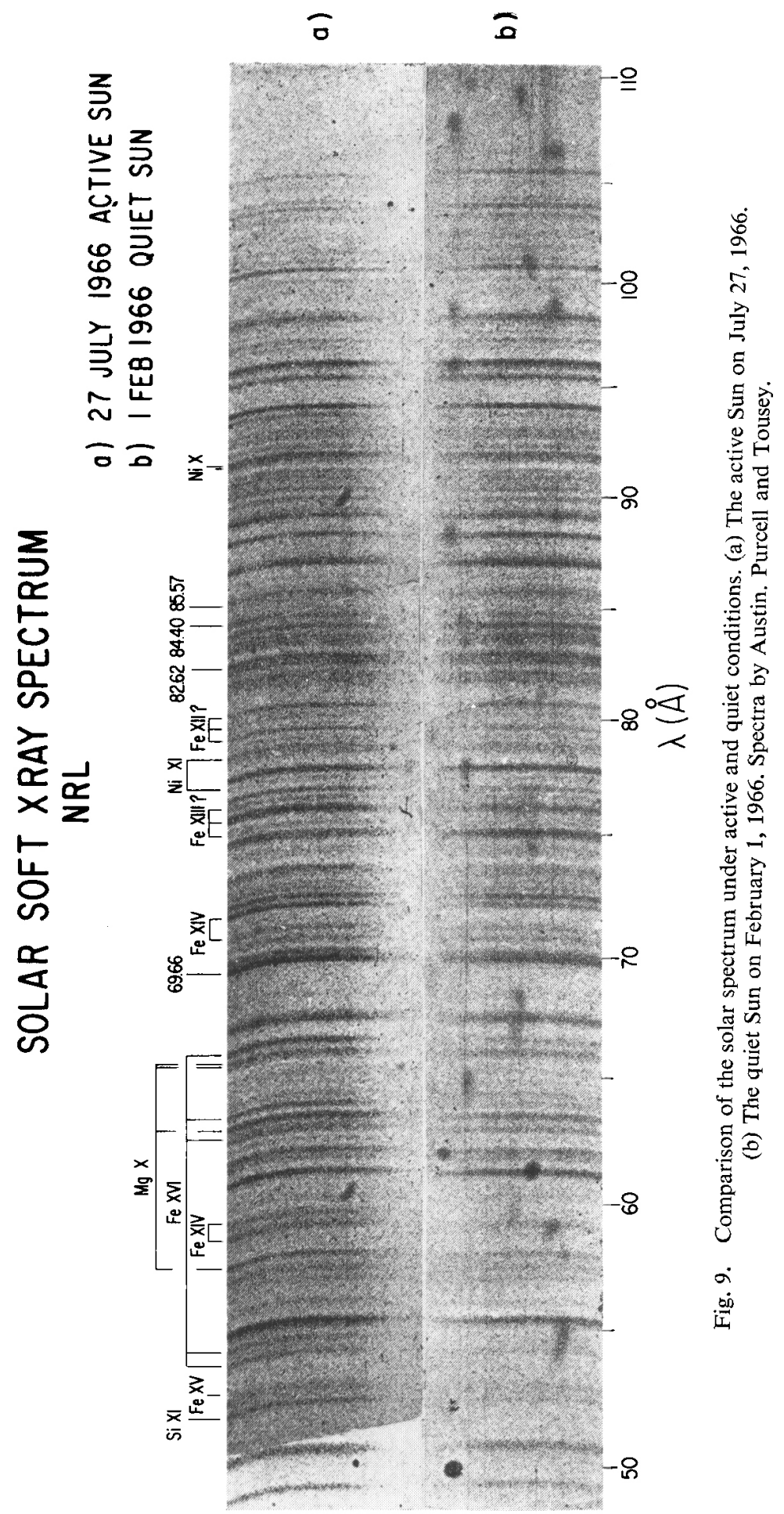


the flights of the scanning monochromators of the AFCRL group which covered the wavelength range from 260-1300 $\AA$, although several purely coronal (MgX, SixII, Fexv, and FexvI) lines are observed in this wavelength range. Hall and Hinteregger (1970) have listed the strongest lines observed in these flights, and Hall et al. (1969) have given a complete list of the flights of this instrument through 1968 and discussed the variation of the strongest EUV lines since 1961.

Figure 8 shows the solar spectrum between 33 and $128 \AA$ as observed by Manson $(1966,1967)$ with the AFCRL photoelectric scanning spectrometer. The photoelectric spectra are superior to photographically recorded spectra in calibration, but generally have lower resolution and poorer sensitivity for weak lines. Figure 9 shows two spectra recorded by the NRL group (Austin et al., 1966; Widing and Sandlin, 1968) for the spectral range 33-110 $\AA$, comparing the Sun under active and quiet conditions. Widing and Sandlin have compiled a definitive list of identifications based on the NRL and the 3 November 1965 AFCRL spectra. The wavelength region includes iron lines from the $\mathrm{NaI}$ (FexvI) to the $\mathrm{K}_{\mathrm{I}}$ (FeVIII) isoelectronic sequences, which are excited over a broad temperature range from $4 \times 10^{5} \mathrm{~K}$ to $2 \times 10^{6} \mathrm{~K}$. Silicon lines from the Lir (SiXII) to the NI (Si VIII) isoelectronic sequence have also been identified by Widing and Sandlin. The absence of the lines of SivII, which would be excited at temperatures near $4 \times 10^{5} \mathrm{~K}$, suggests that the $\mathrm{Fe}$ vill radiation may also come primarily from temperatures near $6 \times 10^{5} \mathrm{~K}$, and in fact the model of the solar atmosphere constructed by Pottasch (1967) predicts an order of magnitude less material at $4 \times 10^{5} \mathrm{~K}$ than at $6 \times 10^{5} \mathrm{~K}$. Spectra with higher sensitivity should reveal the lines of less highly ionized silicon and iron. For the lighter ions, magnesium and neon, one observes fewer stages of ionization in the wavelength range below $110 \AA$. The resonance lines of O VI (Lii), for example $2 s-3 p$ and $2 p-3 d$, are at $150-170 \AA$ in the wavelength range from $110-160 \AA$ in which high quality photographic and high resolution photoelectric

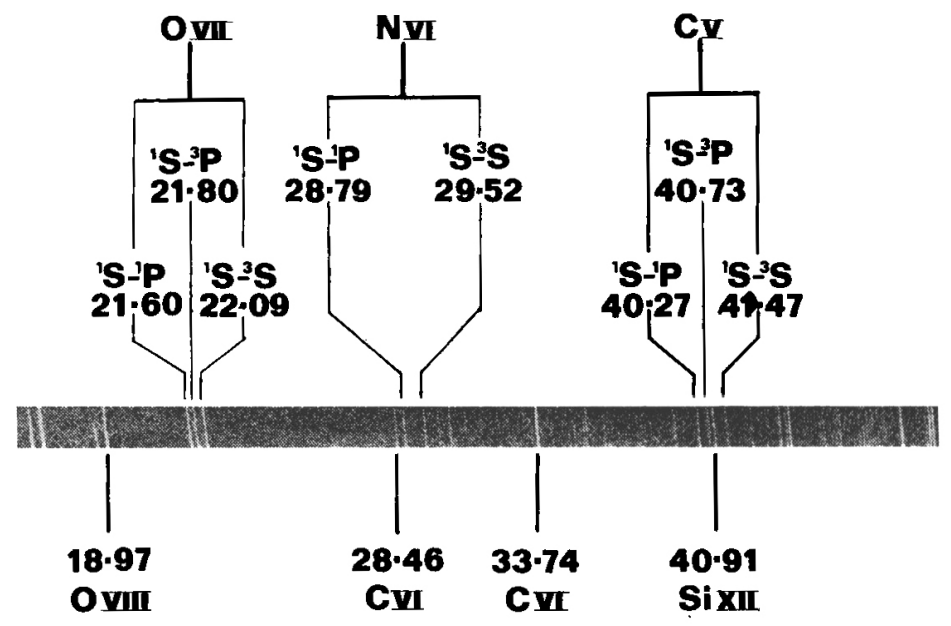

Fig. 10. Spectrum of the Sun between 15 and $60 \AA$. (Courtesy of B. B. Jones) 


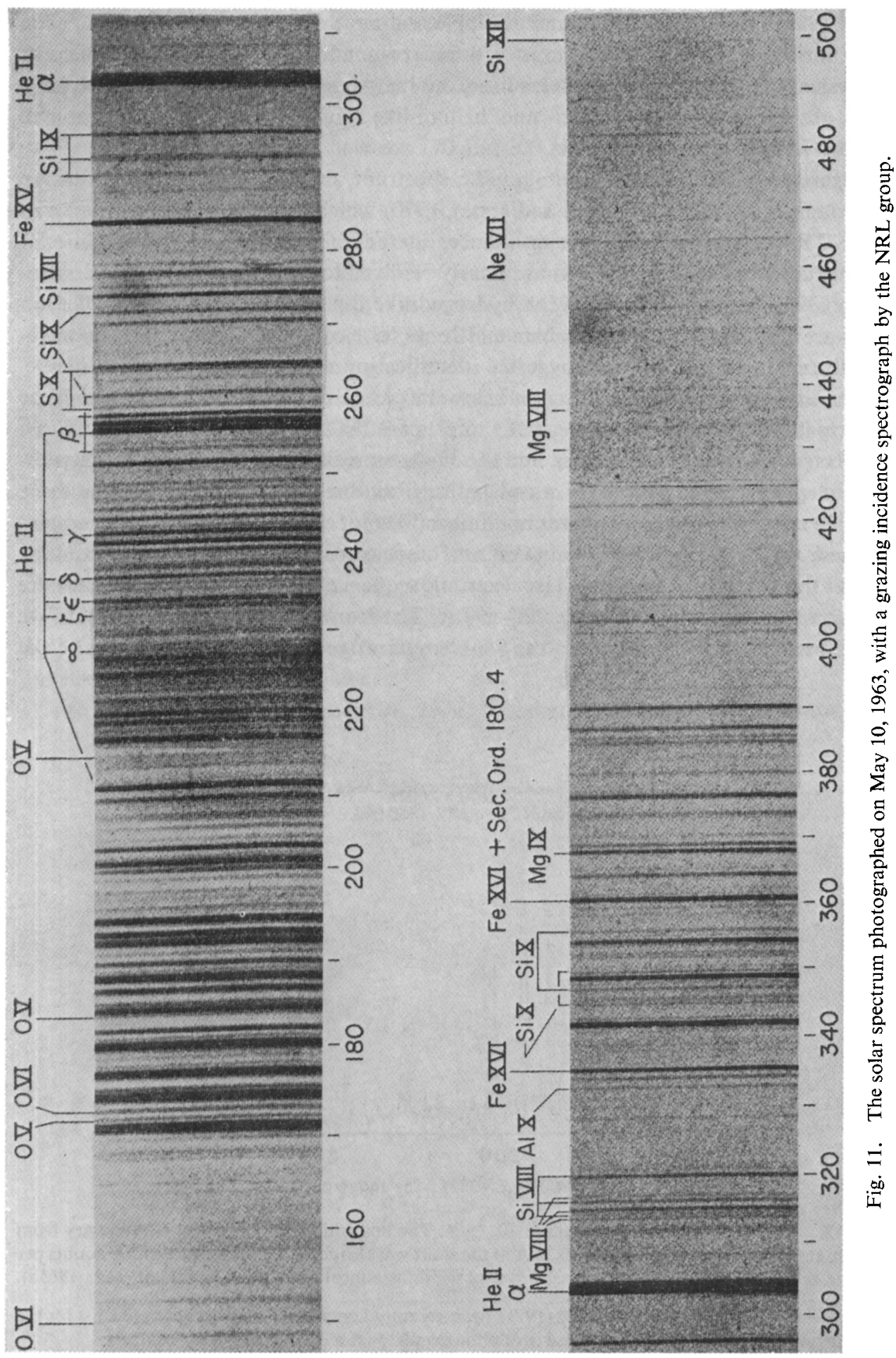


spectra have only recently become available and have not been fully analyzed. ${ }^{*}$ The observation of the lines due to ions of the corresponding isoelectronic sequences of the less abundant elements, nickel, sulfur, calcium, argon and aluminum, which have been observed in their hydrogen- and helium-like stages of ionization in flares, will require higher sensitivity spectra.

Figure 10 shows a recent photographic spectrum obtained by the Culham group (Freeman et al., 1971; Freeman and Jones, 1970) which has high sensitivity down to $15 \AA$. The line groups due to the resonance, intercombination, and forbidden lines of the helium-like ions $\mathrm{CV}$ and $\mathrm{NvI}$ are clearly resolved for the first time in these observations. The resonance lines of the hydrogen-like ion $\mathrm{CVI}$, and the lithium-like ion Si XII are also clearly visible. Freeman and Jones have compiled a line list for the wavelength region 15-700 $\AA$, with suggested identification omitted for only a few lines.

The lines in the wavelength region below $150 \AA$ are due to transitions in which the principal quantum number changes. Examples are the $2 s-3 p$ transition in the Lil-F I isoelectronic sequences of silicon, and the $3 p-4 s$ transitions in the $\mathrm{NaI}-\mathrm{A} I$ isoelectronic sequences of iron. At longer wavelengths, lines due to transitions from the same ions, in which the principal quantum number does not change, predominate. Examples of these are the $2 s-2 p$ transitions in the Li I-F I isoelectronic sequence of silicon, and the $3 p-3 d$ transitions in the $\mathrm{Na}$ I-A I isoelectronic sequences of iron. The silicon lines are in the wavelength range from $\sim 220-500 \AA$. The iron lines, whose identification we have discussed previously, are in the wavelength range from $170-230 \AA$. Lines of the

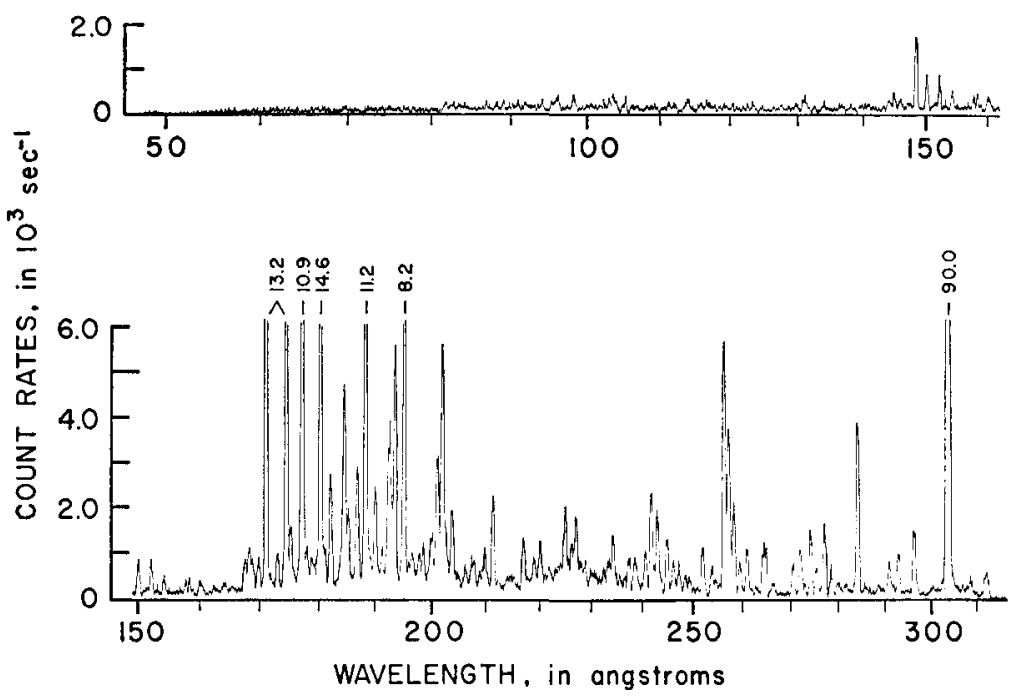

Fig. 12. Solar XUV spectrum of March 30, 1964. The line widths are instrumental and vary from $0.75 \AA$ at the long wavelength end to $0.25 \AA$ at the short wavelength end. Any feature of 200 counts per second or more, and of the proper instrumental width, is almost certainly a line (Hall et al., 1965a).

* Note added in proof: Behring et al. (1971) have recently compiled a very complete line list for the wavelength region from 60-385 $\AA$ based on a photograph spectra obtained on May 16, 1969. 
ions of several stages of ionization of the lighter elements ( $\mathrm{Ne}, \mathrm{Mg}$ ) of the type $2 s-2 p$ are also observed in the normal incidence region beyond $\sim 500 \AA$, and a number of lines of $\mathrm{OV}$, and $\mathrm{OVI}$ of the type $2 s-2 p$ and $2 p-2 d$ are also prominent in this wavelength region.

Figure 11 is solar spectrum between 170 and $500 \AA$, as observed by the NRL group (Austin et al., 1966) on May 10, 1963. The photoelectric spectrum obtained by Hinteregger and his co-workers at AFCRL (Hall et al., 1965a) is shown in Figure 12. The identification of the Si VII to SiXII lines in the spectrum between 220 and $500 \AA$ has been discussed by Widing and Porter (1965), and of the FeIX-FexVI lines between 170 and $230 \AA$ has been discussed by Gabriel et al. (1966), Fawcett et al. (1967), and Fawcett et al. (1968). Pottasch (1967) has tabulated the lines observed, and their intensities, with emphasis on the region above $170 \AA$.

Figure 13 is a spectrum obtained by the scanning grazing incidence monochromator fiown by the GSFC group on OSO 1 in 1962. These observations were critical in identifing the FeIX-Fexvi lines, because the long term satellite observations were able to observe the increasing modulation of the flux in the lines of the more highly ionized ions with solar activity. Neupert (1967) has described the variation of the line intensities for these stages of ionization, from the transition region ion Fe IX to the pure coronal ion FexvI.

The recent spectrum of the wavelength region from 140-330 $\AA$ by the Culham group for March 20th of 1968 is shown in Figure 14. The domination of the spectrum by the intense iron lines is clearly evident.

The Culham group (Freeman et al., 1971; Freeman and Jones, 1970) has now pho-

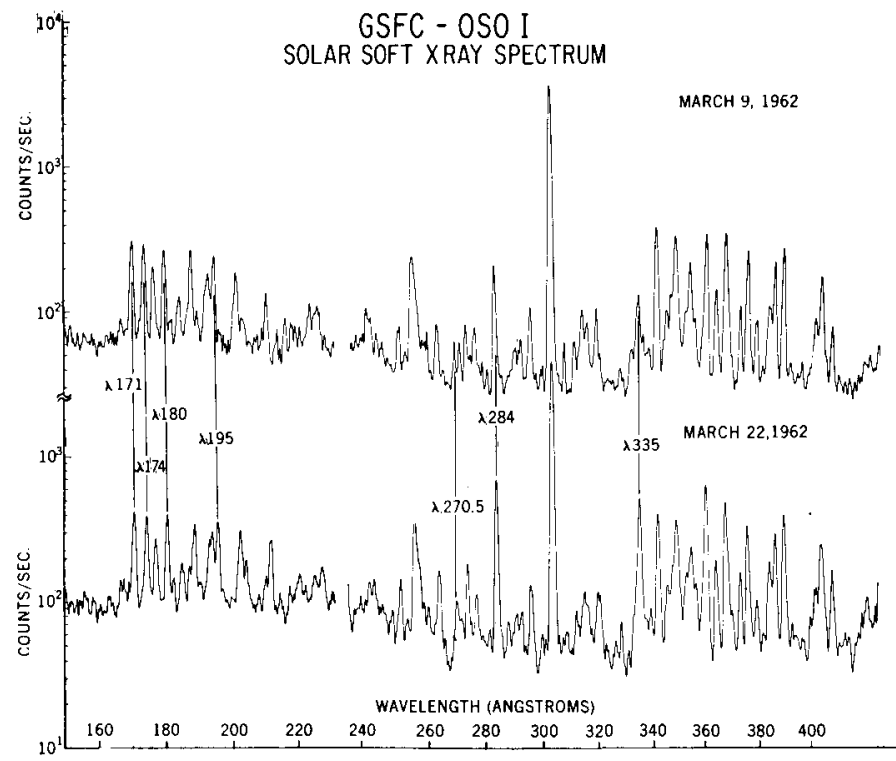

Fig. 13. Solar spectrum on two days as observed on OSO1 by the GSFC group. 


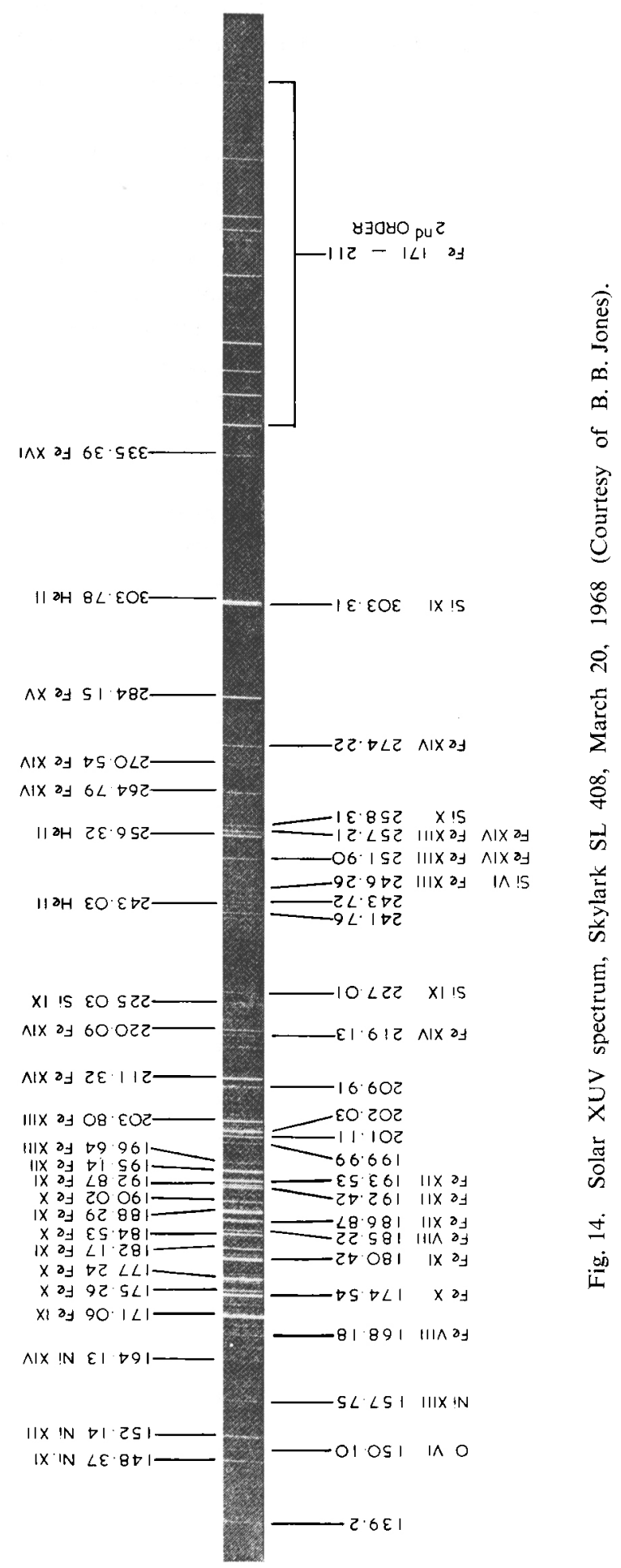


tographed the complete coronal spectrum from 15-700 $\AA$, using grazing incidence grating spectrometers. These spectra, obtained near the time of solar maximum, complement the earlier grazing incidence grating spectra, over the range 30-500 $\AA$, obtained by the NRL group, with photographic techniques and by the AFCRL group with photoelectronic techniques.

\subsection{The SPATIAL Distribution of THE CORONAL EMISSION}

Spectroheliograms with high spectral resolution are available for coronal emission lines only at wavelengths above $\sim 300 \AA$. The difficulty of grazing incidence imaging techniques has resulted in the concentration on broad band imaging of the X-ray corona, and very high quality images have resulted (Underwood and Muney, 1967; Vaiana et al., 1968). The coronal emission, particularly for the lines formed at high temperature, is highly concentrated in small active regions which can have dimensions of $1^{\prime}$ or less. The absence of spatial resolution in coronal spectral observations complicates their analysis and interpretation.

Spectroheliograms, which resolve a single emission line, for the transition region and lower corona have been obtained by the Naval Research Laboratory on rockets (Tousey, 1971) and by the Harvard College Observatory group on OSO 4 and OSO 6. Noyes (197I) has reviewed both the NRL and HCO results. An atlas of the OSO-4 results has been published by Reeves and Parkinson (1970). The rocket spectroheliograms of the NRL group were obtained with a prototype of the ATM spectroheliograph which will be launched in 1973, and are discussed in Section 5.

The X-ray telescope experiment on the OSO-4 spacecraft by a group at American Science and Engineering (Krieger et al., 1971), has obtained images in the 2.5-12 $\AA$ band, and has studied the morphology and evolution of active regions in detail. The University of Leicester and the University College London have flown a unique spectroheliograph on the OSO-5 satellite (Herring et al., 1971). This instrument uses a grazing incidence parabolic reflector which achieves a $2^{\prime}$ resolution on the disk, and a proportional counter detector. The resolution of this detector allows the bandpass of the instrument to be limited to a wavelength interval which is strongly influenced by one, or a few emission lines. Parkinson and Pounds (1971) have reported on the spectroheliograms obtained with this instrument. Simultaneous observations with the UCL/UL OSO-5 spectroheliograph and with a high resolution X-ray spectrometer would greatly simplify and enhance the analysis of both observations.

\subsection{IDENTIFICATION OF SPECTRAL LINES}

The identification of lines in the coronal spectrum has recently been reviewed by Gabriel (1970). As Gabriel points out, coronal conditions depart significantly from LTE, and therefore forbidden lines, intersystem lines, and lines from autoionizing levels are observed with intensities comparable to those in permitted lines. Furthermore, the factors governing the relative intensities of these lines may allow the direct determination of some of the physical parameters of the coronal plasma. On the other hand, some of the lines which have been observed in the corona cannot be observed 
in laboratory plasmas, and it is difficult to establish standard wavelengths with which the coronal lines can be compared.

The most important aids in identifying permitted lines and intersystem lines are experimental studies in the laboratory and theoretical studies of the spectra of the highly ionized ions which are dominant in the solar spectrum. In addition to the standard reference works by Moore $(1949,1952)$ and Kelly $(1968)$, there are a number of recent results which are of particular interest for the lines of the hydrogen- and helium-like ions of the heavier elements, and for the lines of the lithium-like to neon-like ions of the medium elements $(\mathrm{Mg}, \mathrm{Al}, \mathrm{Si}, \mathrm{S})$. We have tabulated references to these results in Table III. We have already pointed out the critical importance of laboratory studies of the spectra of the NaI to ArI sequences of iron to the identification of the lines which dominate the coronal spectrum between 170 and $220 \AA$.

In the case of forbidden lines, the various competing processes which may depopulate the metastable level in question must be considered before positive identifications can be made. An important example is the decay of the $2^{3} S_{1}$ level in helium-like ions,

TABLE III

Theoretical and laboratory references for the spectra of coronal ions

\begin{tabular}{|c|c|c|c|}
\hline $\begin{array}{l}\text { Isoelectronic } \\
\text { sequences }\end{array}$ & Elements & Comments & Reference \\
\hline $\mathrm{H}, \mathrm{He}$ & $\mathrm{Ne}, \mathrm{Ar}$ & Experimental & Peacock et al. (1969) \\
\hline $\mathrm{H}, \mathrm{He}$ & $\mathrm{C}, \mathrm{N}, \mathrm{O}$ & Experimental & Roth and Elton (1968) \\
\hline $\mathrm{He}, \mathbf{L i}, \mathrm{Be} \mathbf{B}$ & $\mathrm{Fe}$ & Theoretical & Chapman (1969) \\
\hline $\mathrm{H}, \mathrm{He}$ & $\begin{array}{l}\text { Ti, V, Cr, Mn, Fe, } \\
\mathrm{Ni}\end{array}$ & Experimental & Cohen et al. (1968) \\
\hline $\mathrm{He}-\mathrm{F}$ & $\mathrm{Li}-\mathrm{Ni}$ & Theoretical & Vainshtein and Sefranova (1971) \\
\hline Li & $\mathrm{Mg}, \mathrm{Al}, \mathrm{Si}$ & Experimental & Feldman et al. (1970) \\
\hline $\mathrm{Li}, \mathrm{Be}$ & $\mathrm{Si}$ & Experimental & Tondello (1969) \\
\hline $\mathrm{Be}$ & $\mathrm{Mg}, \mathrm{Al}, \mathrm{Si}$ & Experimental & Hoory et al. (1970) \\
\hline $\mathrm{Li}-\mathrm{N}$ & $\mathrm{P}, \mathrm{S}$ & Experimental & Fawcett et al. (1970) \\
\hline $\mathbf{L i}-\mathbf{N}$ & $\begin{array}{l}\mathrm{Na}, \mathrm{Mg}, \mathrm{Al}, \mathrm{Si}, \mathrm{P} \\
\mathrm{S}, \mathrm{Cl}\end{array}$ & Experimental & Fawcett (1970) \\
\hline $\mathrm{Li}-\mathrm{Ne}$ & Ar & Experimental & Connerade et al. (1971) \\
\hline $\mathrm{Li}-\mathrm{Na}$ & $\mathrm{Fe}, \mathrm{Ti}$ & Experimental & Lie and Elton (1971) \\
\hline $\mathrm{Li}-\mathrm{Na}$ & $\mathrm{Fe}$ & Experimental & $\begin{array}{l}\text { Feldman and Cohen (1968) } \\
\text { Feldman and Cohen (1970) }\end{array}$ \\
\hline $\mathrm{Be}-\mathrm{F}$ & $\mathrm{B}-\mathrm{Fe}$ & Experimental & Fawcett (1971a) \\
\hline $\mathrm{Be}-\mathrm{F}$ & $\mathrm{Si}-\mathrm{Cl}$ & Experimental & Fawcett et al. (1971) \\
\hline $\mathrm{Be}-\mathrm{Ne}$ & $\mathrm{Fe}$ & Theoretical & Connerade (1971) \\
\hline B & $\mathrm{Mg}, \mathrm{Al}, \mathrm{Si}$ & Experimental & Hoory et al. (1971) \\
\hline $\mathrm{C}-\mathrm{F}$ & $\mathrm{Ca}-\mathrm{Fe}$ & Experimental & Fawcett (1971b) \\
\hline $\mathrm{O}$ & $\mathrm{Sc}, \mathrm{Ti}, \mathrm{V}$ & Experimental & Goldsmith et al. (1971) \\
\hline $\mathrm{O}-\mathrm{Mg}$ & $\mathrm{Fe}$ & Experimental & Connerade et al. (1970) \\
\hline $\mathrm{F}$ & $\begin{array}{l}\mathrm{Sc}, \mathrm{Ti}, \mathrm{V}, \mathrm{Cr}, \mathrm{Mn} \text {, } \\
\mathrm{Fe}, \mathrm{Co}, \mathrm{Ni}, \mathrm{Cu}\end{array}$ & Experimental & Cohen et al. (1968) \\
\hline $\mathrm{Na}-\mathrm{Mg}$ & $\mathrm{Fe}, \mathrm{Co}, \mathrm{Ni}, \mathrm{Cu}$ & Experimental & Feldman et al.(1971) \\
\hline
\end{tabular}

We have limited this compilation to papers published in 1968 or later. 
where especially for the lighter ions, collisional de-excitation, two-photon decay (Bely and Faucher, 1969; Drake et al., 1969), and single-photon decay (Drake, 1971a; Feinberg and Sucher, 1971) can potentially contribute to de-excitation. Gabriel and Jordan (1969a) pointed out that a series of unidentified satellites to the resonance lines of helium-like ions agreed in wavelength with the $1 s^{2}{ }^{1} S_{0}-1 s 2 s{ }^{3} S_{1}$ transition. With the theoretical verification (Griem, 1969) that the single-photon decay was the dominant mode of deexcitation they developed a complete theory for the relative intensity of the ${ }^{3} S_{1}$ and ${ }^{3} P_{2 / 1}$ lines which are coupled by collisional transfer (Gabriel and Jordan 1969a, 1970). The helium-like sequence provides another example of a forbidden decay in the magnetic-quadrupole transition $1 s^{2}{ }^{1} S_{0}-1 s 2 p{ }^{3} P_{2}$, which becomes dominant over the allowed transition $1 s 2 s^{3} S_{1}-1 s 2 p{ }^{3} P_{2}$ for large values of $z$ (Garstang, 1969a; Drake, 1969, 1971b). Doschek et al. (1971c) have demonstrated the presence of this line in the solar spectrum in Caxix.

An example of a forbidden decay in the Ner isoelectronic sequence is the decay of the $2 p^{5} 3 s^{3} P_{2}$ state in FexviI, which is the lowest excited level of Fexvil, and consequently is metastable. Garstang (1969a) has pointed out that the $2 p^{5} 3 s^{3} P_{2}$ state should decay by a magnetic quadrupole transition $2 p^{6}{ }^{1} S_{0}-2 p^{5} 3 s^{3} P_{2}$. Walker and Rugge (1970) have observed this line in the corona (Figure 15) where its presence is clearly

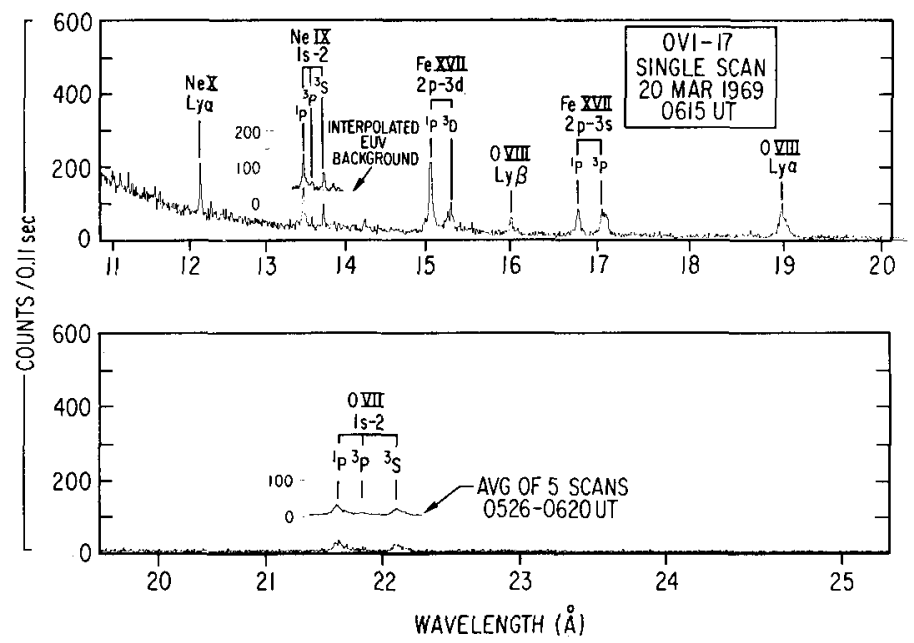

Fig. 15. Solar spectrum between 12 and $23 \AA$. The presence of the FexvIII $2 p^{6}{ }^{1} S_{0}-2 p^{5} 3 s^{3} P_{2}$ line 17.10 $\AA$, near the $2 p^{6}{ }^{1} S_{0}-2 p^{5}{ }^{3} P_{1}$ line at $17.05 \AA$ is demonstrated by the width of the profile of the feature at $17.07 \AA$ compared to the profile of the $2 p^{6} S_{0}-2 p^{5} 3 s^{1} P_{1}$ line at $16.77 \AA$.

demonstrated by the difference in profile of the $2 p^{6}{ }^{1} S_{0}-2 p^{5} 3 s^{1} P_{1}$ (16.77) line, and $2 p^{6}{ }^{1} S_{0}-2 p^{5} 3 s^{3} P_{1}$ (17.051) and $2 p^{6}{ }^{1} S_{0}-2 p^{5} 3 s^{3} P_{2}$ (17.010) multiplet. This line is particularly interesting since the $3 p^{5} 3 s$ states are populated chiefly by cascade from the $2 p^{5} 3 p$ levels (Beigman and Urnov, 1970).

The recent identification of satellite lines in the coronal spectrum resulting from 
the radiative decay of autoionizing levels (Walker and Rugge, 1970; Neupert and Swartz, 1971) has resulted in considerable interest in these lines, and an appreciation of the importance of the resonance process of dielectronic recombination in forming the coronal spectrum. Walker and Rugge (1971b) have identified a number of lines from autoionizing levels in both helium-like ions (hydrogen-like line satellites) and lithium-like ions (helium-like line satellites). Four of the lithium-like satellite lines identified by Walker and Rugge are shown in Figures $16 \mathrm{a}$ and 16b. The satellite line

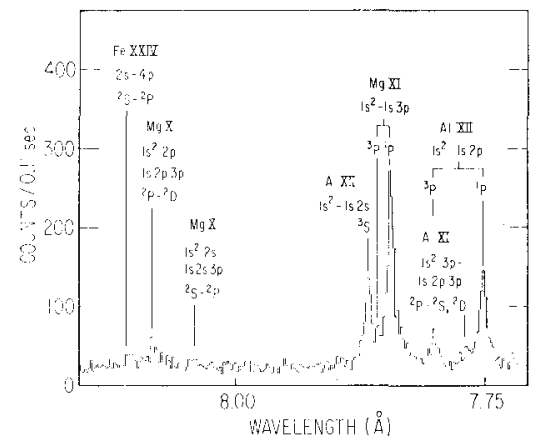

(A)

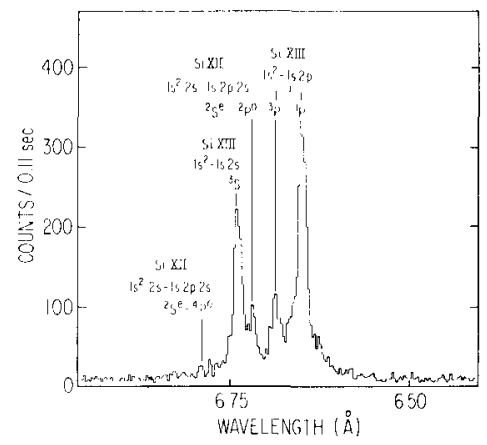

(B)

Figs. 16a-b. Enlargement of the solar spectra observed with the OV1-17 satellite on March 20, 1969. Several autoionizing satellite lines from SiXII and $\mathrm{Mgx}$ are shown. The spin forbidden $1 s^{2}{ }^{1} S_{0^{-}}$ $-1 s 3 p^{3} P_{1}$ line in $\mathrm{MgXI}$ is resolved from the $1 s^{2}{ }^{1} S_{0}-1 s 3 p^{1} P_{1}$ line in this spectra.

$1 s^{2} 2 s^{2} S-1 s 2 s 2 p^{2} P$ shown in Figure $16 \mathrm{~b}$ for SiXII is $16 \%$ as strong as the resonance line of SiXIII. The lithium-like autoionizing satellite lines become as strong as the helium-like forbidden line for the high $z$ ions such as iron. Walker and Rugge (1971b) have discussed the experimental laboratory observations and the theoretical treatments of these satellite lines in some detail, and present a rather complete set of references. Recently, a number of authors (Neupert, 1971; Doschek et al., 1971a, b; Grineva et al., 1971) have reported the observations of a substantial number of lithium-like satellite lines during flares, when the lines of the heavier ions are at high levels of intensity. Neupert has also identified satellite lines due to $1 s-2 p$ transitions in beryllium-like ions.

There are still some questions concerning the identification of the permitte dlines of Fe XVIII in the spectrum below $25 \AA$. The lines of Fe XVIII have been tentatively identified in active regions in the corona by Evans et al. (1967) and by Walker and Rugge (1969). These identifications were based on the laboratory spark spectra of Fawcett et al. (1967) and of Feldman and Cohen (1968). Subsequently, Cohen et al. (1968) have published a more complete study of the FI isoelectronic sequence, and Connerade et al. 1970) have questioned a number of the identifications of FexvII-FexxIv lines made by Feldman and Cohen. The inclusion of possible cascade effects from the $2 p^{4} 3 p$ configuration of FexviII could, for example, alter the relative line intensities within the 
$2 p^{4} 3 s$ and $2 p^{4} 3 d$ multiplets which are predicted on the basis of the oscillator strengths connecting these levels to the ground level.

\subsection{ABSOLUTE INTENSITY MEASUREMENTS}

Consistent and reliable absolute intensity XUV fluxes of spectral lines, and of the continum, are indispensible for a quantative investigation of the solar corona. The earliest absolute line fluxes were obtained by the AFCRL group (Hintregger et al., 1964; Hall et al., 1965a, 1965b) in the range $60 \AA-1000 \AA$, and by groups at NRL (Austin et al., 1966 ) in the range $30 \AA-500 \AA$ and (Blake et al., 1965) in the range $13 \AA$ to $25 \AA$. Pottasch, in a series of papers analyzing these results (Pottasch 1963, 1964a, b, 1966, 1967), demonstrated that the coronal spectrum required an iron abundance an order of magnitude higher than the then current photospheric abundance, in agreement with earlier results on coronal forbidden lines (Wooley and Allen, 1948).

The results of more recent observations, using grazing incidence techniques, have been presented by Manson $(1968,1972)$ for the region $30 \AA-128 \AA$, by the NRL group (Austin et al., 1967, Widing and Sandlin, 1968) for the region $30 \AA-110 \AA$, and by the AFCRL group (Hall and Hinteregger, 1970; Hall et al., 1969). All of the absolute flux measurements, with the exception of the recent OSO-3 results of the AFCRL group, are due to observations made with rocket spectrometers. Widing and Sandlin have analyzed some of these more recent results, to provide a model which is in general agreement with that of Pottasch. Most recently, the Culham group (Freeman and Jones, 1970) have reported absolute intensity measurements of the line spectrum over a broad wavelength range ( $15 \AA-700 \AA)$, based on the observations obtained in a series of rocket flights.

At shorter wavelengths, absolute line fluxes have been reported by the Leicester University Group (Evans and Pounds, 1968; Batstone et al., 1970), and by the Los Alamos Group (Evans et al., 1966, Argo et al., 1968) from rocket observations with Bragg crystal spectrometers. Absolute fluxes obtained with satellite borne crystal spectrometers have been reported by The Aerospace Corporation group (Walker and Rugge, 1968; Walker and Rugge, 1969). The Leicester and Aerospace results have been analyzed by Beigman and Vainshtein (1970), Batstone et al. (1970), and by Chambre (1971).

Comparing the absolute line fluxes reported by various observers is difficult, due to the substantial variation of the coronal spectrum, with solar activity. This is an especially serious problem for the spectral range below $100 \AA$, which is dominated by lines formed in active regions.

Hinteregger (1970) discusses this problem, and presents more recent observations taken by Manson during a period of higher solar activity. In general, our knowledge of the absolute photometry of the 'true coronal' lines (with the possible exception of the strong Fexvi and Fexv lines at $335.4 \AA$ and $284.1 \AA$ ) below $400 \AA$ is not in a satisfactory state. The more recent results of Freeman and Jones, obtained in 1969 indicate fluxes roughly twice those measured by Manson and Austin et al., obtained in 1965, 1966, and 1967. The Culham results are also larger than those reported by Evans 


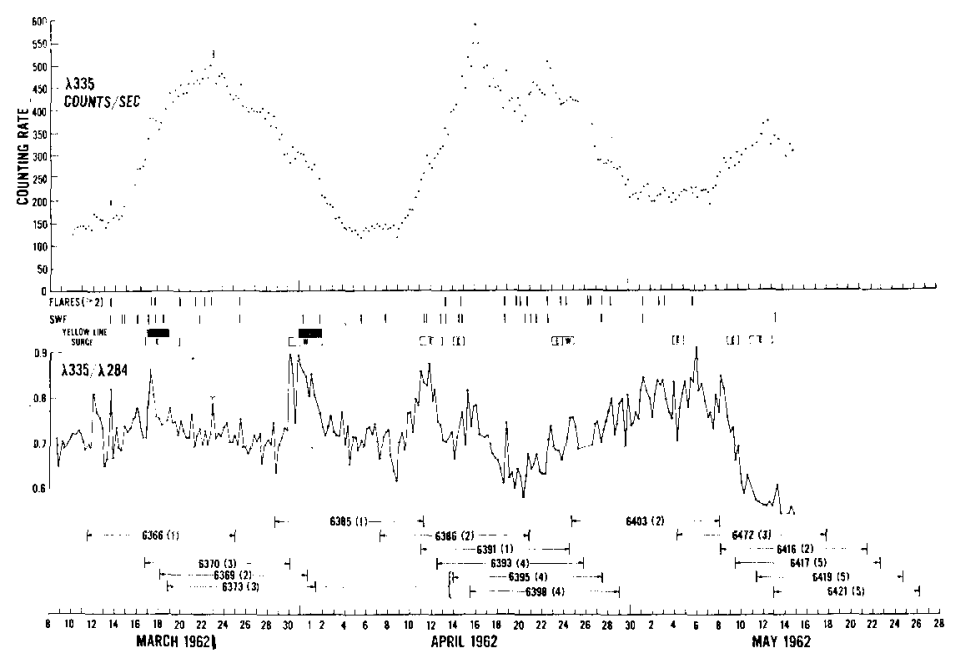

Fig. 17. Variation of the Fexvi and Fexv coronal lines with solar activity as observed on OSO1.

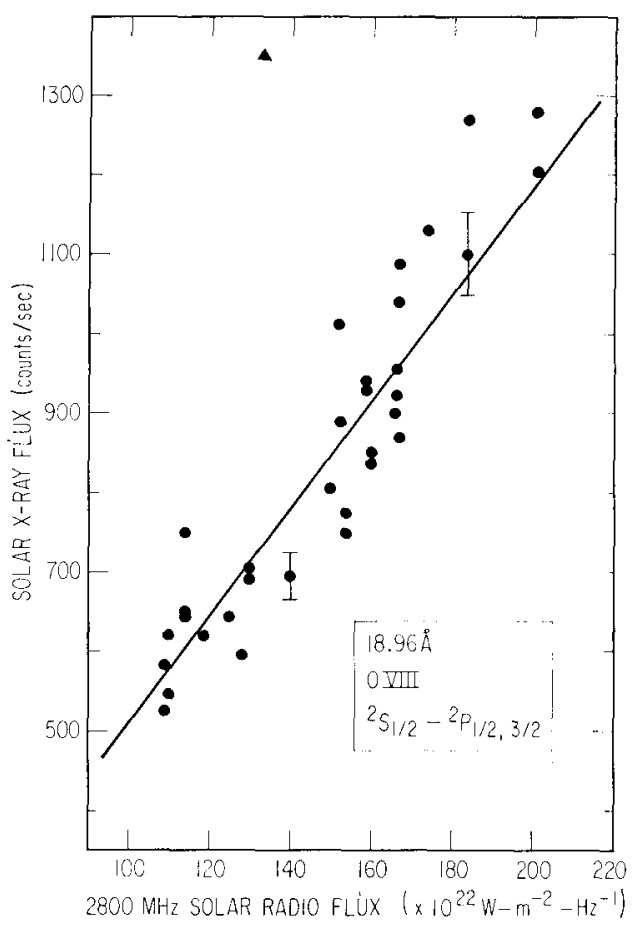

Fig. 18. Variation of the OVIII Lyman- $\alpha$ line with solar radio flux as observed on OVI-10. 
and Pounds and by Walker and Rugge, obtained in 1967 (the fluxes given by the Leicester and Aerospace groups are in excellent agreement). In view of substantial short term as well as long term variations at wavelengths below $25 \AA$, it is difficult to assess the significance of a detailed comparison. Figure 17 shows the variation of the absolute and relative counting rates in the Fexvi and Fexv lines as observed by OSO 1 (Neupert, 1967). These lines are formed at temperatures between 2.5 and 4 million deg. Rugge et al. (1970) and Rugge and Walker (1971a) have discussed the variation of soft $\mathrm{X}$-ray line fluxes with solar activity, and have demonstrated that for observations taken in the absence of flare activity, line fluxes from the ions $\mathrm{O}$ VII, O VIII and FexvII are well correlated with the daily $2800 \mathrm{MHz}$ solar radio flux. The correlation for the Ovir Lyman- $\alpha$ line is shown in Figure 18 (Rugge et al., 1970. These results suggest that the comparison of XUV observations obtained at different times can be accomplished with the aid of data from solar radio patrols.

Absolute fluxes for the X-ray continuum are rare. Manson (1966) has presented some values for the spectral region under $100 \AA$. Evans and Pounds (1968), and Walker and Rugge (1969), have presented data for the region between 16 and $25 \AA$. Walker and Rugge (1971a) have recently analyzed the continuum between 9 and $23 \AA$, using OV $1-10$ results. The comparison between the observed continuum flux and that computed from a model derived by analyzing the line fluxes is presented in Figure 26. Details of this model will be presented in the section on coronal temperature models.

In summary, we see that absolute line and continuum flux data are rare. This is due to the difficulty of maintaining good calibrations on satellite instrumentation, and in fact with the exception of results from the AFCRL monochromator on OSO 3, and from The Aerospace Corporation spectrometer on OV1-10, all of the results published in the literature are from observations made with rocket borne instruments.

Hinteregger (1970) has given the most complete review of the problem of absolute solar line fluxes, and has given a table of recommended fluxes for the strongest lines, and integrated fluxes for spectral bands.

Dupree and Reeves (1971) have recently compiled a list of absolute fluxes for the quiet equatorial spectrum between 304 and $1394 \AA$, based on OSO-4 observations.

\section{Theoretical Basis for the Interpretation of the Observed Spectra}

In this section we shall present a brief discussion of the fundamental atomic processes which shape the coronal spectrum. Rates for these atomic processes must be known before an interpretation of the spectrum can be undertaken. A knowledge of these atomic rates, together with the experimental determination of the absolute flux in emission lines and in the continuum, forms the basis for the determination of the properties of the coronal plasma. We shall discuss the theoretical computation of the coronal spectrum in sufficient detail to present graphical results for the spectrum below $25 \AA$, as a partial basis for our discussion of coronal models in the next section, and to illustrate the relative importance of different processes in forming the spectrum.

In a recent review on the corona, Zirker (1971) has commented, "Considering the 
effort (and expense) invested in obtaining adequate observations [of the coronal spectrum], especially from satellites, it is unfortunate that so little effort is expended in calculating atomic parameters." Although (or perhaps because) the present reviewer is responsible for some of those (expensive) satellite observations, he strongly endorses Zirker's sentiments.

\subsection{The population of EXCited STAtes}

Pottasch $(1963,1964)$ has discussed approximate methods for permitted lines in the coronal spectrum, based on the Bethe approximation method of Van Regemorter (1962). More recently, several authors have calculated the coronal line spectrum between 1 and $100 \AA$ (Landini and Monsignori Fossi, 1970; Tucker and Koran, 1971; Mewe, 1972a,b; Beigman and Vainshtein, 1967a; and Beigman et al., 1971). Tucker and Koran, in particular, have presented a rather complete discussion of the excitation processes of importance and have presented tabular results for the spectrum between 1 and $80 \AA$ for a broad range of temperatures.

The population of excited levels in the solar corona can occur by a number of processes. These processes include electron collisional excitation, cascade from higher levels, dielectronic recombination (this process populates doubly excited levels and can, as we shall see, result both in satellite lines and in photons which are unresolvable from resonance lines), and radiative recombination. Two other processes, inner shell excitation and direct double excitation may contribute to the population of doubly excited levels during flares, but are unimportant for the quiet Sun. Proton excitation is not important except when $k T$ is large compared to the excitation energy. It is therefore unimportant in the coronal XUV spectrum, except in the case of collisional coupling between fine structure levels, where one level is metastable. An example is $1 s 2 s^{3} S$ in helium-like ions (Blumenthal et al., 1971).

The calculations of Pottasch, of Landini and Monsignori Fossi, and of Mewe, have used the Bethe approximation method of Van Regemorter*, and these authors have tabulated the oscillator strengths required for this method. However, this method underestimates the intensity of transitions, including allowed transitions, from upper levels which are not connected to the ground level by allowed transitions. An example is provided by the strong quadrapole excitation strength to the $3 \mathrm{~d}$ level in lithium-like ions (Bely and Petrini, 1970).

The calculation of electron collision excitation cross sections has recently been reviewed by Seaton (1969), and by Bely and Van Regemorter (1969). Bely and Van Regemorter present references to published theoretical computations in a convenient tabular format for ions of each isoelectronic sequence.

We shall consider hydrogen-like systems first. Beigman et al. (1969) have calculated, in the Coulomb-Born approximation, excitation cross sections for a wide range of excitations for ions of the isoelectronic sequences from hydrogen to neon. Tucker and Koran use their results for hydrogen-like ions. We prefer to use the Coulomb-Born

* Van Regemorter has modified the Bethe approximation by deriving effective Gaunt factors for optically allowed transitions. 
Oppenheimer results of Burgess et al. (1970), who have computed cross sections for the $1 \mathrm{~s}-2 \mathrm{~s}$ and $1 \mathrm{~s}-2 \mathrm{p}$ transition in hydrogen-like ions. They express the cross sections in terms of a normalized collision strength $z^{2} \Omega$

$$
\sigma=\frac{\pi a_{0}^{2} \mathrm{Ry}}{\left(2 j_{i}+1\right) E} \frac{z^{2} \Omega}{z^{2} \mathrm{Ry}},
$$

where $E$ is the electron energy, Ry the rydberg, $z$ the nuclear charge, $a_{0}$ the Bohr radius, and $j_{\mathrm{i}}$ the initial ion spin. We define the temperature averaged collision strength

$$
\overline{z^{2} \Omega(\theta)}=\int_{0.75}^{\infty} z^{2} \Omega \exp [-(\varepsilon-0.75) / \theta](\mathrm{d} \varepsilon / \theta),
$$

where

$$
\theta=k T / z^{2} \mathrm{Ry}=3 k T / 4 E_{\mathrm{Ly}-\alpha} \quad \varepsilon=E / z^{2} \mathrm{Ry}=3 E / 4 E_{\mathrm{Ly}-\alpha} .
$$

The quantity $z^{2} \Omega(0)$ is tabulated for the $1 s-2 p$ and $1 s-2 s$ transitions in Table IV. We

\begin{tabular}{|c|c|c|c|c|c|}
\hline 0 & $\overline{z^{2} \Omega} 1 s-2 p$ & $\overline{z^{2} \Omega} 1 s-2 s$ & $\theta$ & $\overline{z^{2} \Omega} 1 s-2 p$ & $\overline{z^{2} \Omega} 1 s-2 s$ \\
\hline 0.1 & 1.56 & 0.365 & 0.60 & 2.02 & 0.376 \\
\hline 0.15 & 1.60 & 0.367 & 0.65 & 2.06 & 0.377 \\
\hline 0.20 & 1.66 & 0.368 & 070 & 2.09 & 0.377 \\
\hline 0.25 & 1.71 & 0370 & 0.75 & 2.11 & 0.378 \\
\hline 0.30 & 1.76 & 0.371 & 0.80 & 2.14 & 0.378 \\
\hline 0.35 & 1.81 & 0.372 & 0.85 & 2.16 & 0.379 \\
\hline 0.40 & 1.85 & 0.373 & 0.90 & 2.17 & 0.379 \\
\hline 0.45 & 1.90 & 0.374 & 0.95 & 2.19 & 0.380 \\
\hline 0.50 & 1.94 & 0.375 & 1.00 & 2.20 & 0.380 \\
\hline 0.55 & 1.99 & 0.376 & & & \\
\hline
\end{tabular}

TABLE IV

Temperature averaged hydrogenic collision strengths

can then write the energy radiated by a hydrogen-like coronal ion in its Lyman- $\alpha$ line seen at the Earth as

$$
\begin{array}{r}
\frac{\mathrm{d}^{2} \phi_{\mathrm{ex}}^{\mathrm{Ly}-\alpha}}{\mathrm{d} t \mathrm{~d} V}=n_{\mathrm{e}} n_{\mathbf{H}} A_{z} \frac{n_{i}}{n_{z}} 5.01 \times 10^{-44} \frac{}{z^{2} \Omega_{2 \mathrm{p}}(\theta)} \frac{\exp \left(-E_{\mathrm{Ly}-\alpha} / k T\right)}{\sqrt{T}} \\
\operatorname{ergs~cm}^{-2} \mathrm{~s}(T \text { is in } K),
\end{array}
$$

where $n_{\mathrm{i}} / n_{z}$ is the fraction of the ions of element $z$ in ionization stage $i, A_{z}$ is the relative abundance, and $E_{\mathrm{L} y-\alpha}$ the excitation energy of the Lyman- $\alpha$ line, and $n_{\mathrm{e}}$ and $n_{\mathrm{H}}$ the electron and hydrogen ion densities. Following the method adopted by Tucker and Koran we can compute the excitation rates for higher levels of hydrogenic ions, and for permitted transitions in helium-like ions by scaling cross-sections by the ratio of upper level oscillator strength, $f_{n}$, to upper level excitation energy, $E_{n}$. We shall also modify the two level approximation by introducing the branching ratio, $B_{n n}$, for the 
decay of the upper level $n$. Adopting the convention that quantities with the single subscript $n$ are referenced to the ground level, we can write the energy radiated at the Earth by hydrogenic or helium-like ions for the transition from the upper level $n$ to the lower level $n^{\prime}$ as

$$
\frac{\mathrm{d}^{2} \phi_{\mathrm{ex}}^{n n^{\prime}}}{\mathrm{d} t \mathrm{~d} V}=\frac{f_{n}}{f_{\mathrm{Ly}-\alpha}} \frac{\mathrm{d}^{2} \phi_{\mathrm{ex}}^{\mathrm{Ly}-\alpha}}{\mathrm{d} t \mathrm{~d} V} B_{n n^{\prime}} E_{E_{n}^{\prime}},
$$

where $E_{\mathrm{Ly}-\alpha}$ in Equation (1) has been replaced by $E_{n}$

For the lines of ions of the lithium and neon isoelectronic sequence Bely and his co-workers (Bely, 1966a, b, 1967; Bely and Bely, 1967; Bely and Petrini, 1970) have calculated threshold collision strengths, $\Omega_{n}$. Johnson and Kunze (1971) have measured the collision strengths for a number of transitions in beryllium-like ions, and compared their results with recent calculations by Osterbrock (1970a, b). For lines of these ions, the flux at the Earth can then be written as

$$
\begin{aligned}
& \frac{\mathrm{d}^{2} \phi_{\mathrm{ex}}^{n n^{\prime}}}{\mathrm{d} t \mathrm{~d} V}=n_{\mathrm{e}} n_{\mathbf{H}} A_{z} \frac{n_{\mathrm{i}}}{n_{z}} 6.66 \times 10^{-44} \frac{E_{n n^{\prime}}}{\mathrm{Ry}} \bar{\Omega}_{n} B_{n n^{\prime}} X \times \\
& \times \sqrt{\sqrt{ } T} \mathrm{erg} \mathrm{cm}^{-2} \mathrm{~s} .
\end{aligned}
$$

Tucker and Koran (1971) have tabulated effective collision strengths $\bar{\Omega}_{n}$ for the most important coronal lines between 1 and $80 \AA$ by extrapolating the results of Bely for lithium-like ions to other sequences.

For ions in lower stages of ionization than the neon isoelectronic sequence, which apear at longer wavelengths, Bely and Blaha (1969) have computed collision strengths for some Fexv lines, and Petrini (1969) for some Fexiv lines.

For forbidden lines there are few theoretical treatments available. In addition, competing de-excitation processes must be considered in predicting the strength of forbidden lines. We shall discuss this point in the next section.

The relative population of each ionization stage $\left(n_{\mathrm{i}} / n_{z}\right.$ in Equations (1) and (3)) has been calculated by Jordan $(1969,1970)$ for ions of C, N, O, Ne, Mg, Si, Fe and Ni, and by Allen and Dupree (1969) for ions of C, O, Si and Fe. Beigman et al. (1969) have computed the isoelectronic equilibrium for the helium- and hydrogen-like ions of several abundant elements. These results do not agree well with those of Jordan and Allen and Dupree, due possibly to the neglect of low stages of ionization.* Jordan has included effects of electron density for a coronal model in decreasing the dielectronic recombination rate (see also Burgess and Summers, 1969; Dupree, 1968), and her results have widely used in coronal calculations.**

In most cases cascade from higher levels has not been included in calculations of the coronal spectrum; and the present accuracy of theoretical excitation rates and of ex-

* The results of Beigman et al. (1969) have been revised by Beigman et al. (1971).

** Note added in proof: Landini and Fossi (1971) have calculated ionization equilibrium for ions of $\mathrm{Na}, \mathrm{Al}, \mathrm{A}, \mathrm{K}, \mathrm{Ca}, \mathrm{Cr}, \mathrm{Mn}$, and $\mathrm{Fe}$. 
perimental line fluxes would not appear to warrant the inclusion of this effect. There are, however, some cases in which the effect of cascades is dominant. An example is the $2 p^{5} 3 s$ levels of FexvII, which have a considerably smaller excitation cross section from the $2 p^{6}$ ground state than the $2 p^{5} 3 d$ levels. However, in the corona the line multiplet $2 p^{6}-2 p^{5} 3 s$ is comparable in strength to the multiplet $2 p^{6}-2 p^{5} 3 \mathrm{~d}$. Bely and Bely (1967) have computed the excitation rates to the $2 p^{5} 3 p$ levels, and have shown that while these levels do not have a permitted optical transition to the ground state, they do have a large excitation cross section. The $2 p^{5} 3 \mathrm{~s}$ levels are then populated primarily by cascades from the $2 p^{5} 3 p$ levels. Beigman and Urnov (1970) have computed the relative intensity of the lines from the $2 p^{5} 3 \mathrm{~s}$ and $2 p^{5} 3 d$ levels, and their results are in good agreement with the experimental observations.

Two recombination processes, radiative recombination into excited levels, and dielectronic recombination, can contribute to the population of excited levels. The radiative recombination rate can be calculated by formulas which will be discussed in a later section in connection with recombination processes and the X-ray continuum. For the quiet corona, radiative recombination does not make a significant contribution to the line flux observed in most coronal lines (Tucker and Koran, 1971). In some cases, such as hydrogenic lines from ions which are completely ionized at the most probable coronal temperature, radiative recombination could become an important source of line emission.

Dielectronic recombination, on the other hand, can have an important effect on the coronal spectrum, especially for the more highly charged ions. There are two reasons for this effect. Recombination is relatively more efficient at low temperature as compared to collisional excitation, and the hydrogen- and helium-like ions of the heavier atoms tend to exist at temperatures below their temperature of most efficient collisional excitation in the quiet corona. Further, the doubly excited levels formed in dielectronic recombination can decay either by autoionization or by a radiative transition. Autoionizing probabilities are only weakly dependent on nuclear charge. However, radiative transition probabilities increase rapidly with $z$ so that radiative de-excitation of doubly excited states becomes considerably more important as nuclear charge increases (Walker and Rugge, 1971b).

Lines resulting from the de-excitation of doubly excited levels can appear either as satellite lines to the resonance lines of the next higher stage of ionization, or they can be so close in wavelength to the resonance lines that they effectively increase the flux in the resonance lines. In order to illustrate this point, let us consider transitions from lithium-like ions, which will appear as satellites of helium-like ions. In laboratory plasmas, four satellite lines arising from transitions of autoionizing levels are observed. These transitions have been classified by Gabriel and Jordan (1969a) and are tabulated below (the wavelengths given are for SixIII. Figure 19 shows the appearance of the spectrum of $\mathrm{O}$ VII in the laboratory, and in the corona, with only the strongest lines shown (Gabriel, 1970).*

* Note added in proof: Gabriel and Paget (1972) have analyzed the intensity of the ${ }^{2} S{ }^{2} P$ satellite line for $\mathrm{N} v \mathrm{v}$ and $\mathrm{O}$ vil observed in laboratory plasmas. 

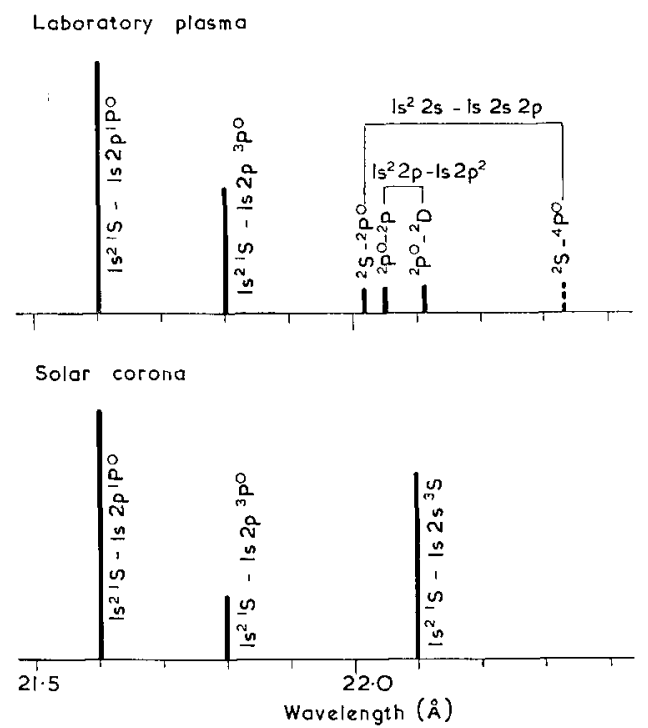

Fig. 19. Spectrum of OvII in the corona and in the laboratory (after Gabriel, 1970).

TABLE V

\begin{tabular}{lll}
\hline & Transition & Si $X I I I$ Wavelength $(\AA)$ \\
1. & $1 s^{2} 2 s^{2} S-\left(1 s 2 p^{3} P\right) 2 s^{2} P^{a}$ & 6.684 \\
2. & $1 s^{2} 2 p^{2} P-1 s\left(2 p^{2}{ }^{1} S\right)^{2} S$ & 6.690 \\
3. $1 s^{2} 2 s^{2} S-\left(1 s 2 p^{1} P\right) 2 s^{2} P^{a}$ & 6.717 \\
4. $1 s^{2} 2 p^{2} P-1 s\left(2 p^{2} D\right)^{2} D$ & 6.740
\end{tabular}

${ }^{2}$ The upper states should more properly be labeled $1 s\left(2 s 2 p^{1} P\right){ }^{2} P$ and $1 s\left(2 s 2 p^{3} P\right)^{2} P$ respectively, but the notation used above makes it more obvious which state has the larger transition probability, and therefore, is strongest in the spectrum.

The longer wavelength ${ }^{2} S-{ }^{2} P$ line has been observed in the corona (see Figure 16b) for Ne vIII and heavier ions. The ${ }^{2} P-{ }^{2} D$ line is too close to the helium-like forbidden line to be resolved in coronal spectra at present.

Ederer et al. (1970) have observed the autoionization spectrum of lithium by photoabsorption. They cannot excite the upper levels associated with transitions 2 and 4 by this method since these levels cannot be reached from the ground level by a dipole transition, however, they have observed autoionizing series which terminate at the upper levels of transitions 1 and 3 . The series with upper levels designated by $\left(1 s 2 p^{1} P\right)$ $n s^{2} P$ is of particular interest since these levels should decay according to

$$
1 s^{2} n s^{2} S-\left(1 s 2 p^{1} P\right) n s^{2} P .
$$

Ederer et al. have measured the excitation energy of the levels $\left(1 s 2 p^{1} P\right) n s^{2} P$. The ener- 
gy of the transition from this level to $1 s^{2} n s^{2} S$ can be calculated according to the formula

$$
E_{1 s^{2} n s-1 s 2 p n s}=E_{1 s^{2} 2 s-1 s 2 p n s}-E_{1 s^{2} 2 s-1 s^{2} n s}
$$

where $E_{1 s^{2} 2 s-1 s 2 p n s}$ is the quantity which has been measured by Ederer et al. The quantity $E_{1 s^{2} 2 s-1 s^{2} n s}$ may be obtained from the tables of Moore (1949). The wavelengths of the transitions from the autoionizing levels in LiI are compared with the wavelengths of the Li II resonance line in Table VI.

\begin{tabular}{lll} 
& \multicolumn{2}{c}{ TABLE VI } \\
Ion & Transition & Wavelength $(\AA)$ \\
\hline & & \\
Li I & $1 s^{2} 2 s^{2} S-\left(1 s 2 p^{1} P\right) 2 s^{2} P$ & 210.46 \\
LiI & $1 s^{2} 3 s^{2} S-\left(1 s 2 p^{1} P\right) 3 s^{2} P$ & 200.32 \\
Li I & $1 s^{2} 4 s^{2} S-\left(1 s 2 p^{1} P\right) 4 s^{2} P$ & 199.61 \\
Li I & $1 s^{2} 5 s^{2} S-\left(1 s 2 p^{1} P\right) 5 s^{2} P$ & 199.40 \\
Li I & $1 s_{2} 6 s^{2} S-\left(1 s 2 p^{1} P\right) 6 s^{2} P$ & 199.31 \\
Li I & $1 s^{2} 7 s^{2} S-\left(1 s 2 p^{1} P\right) 7 s^{2} P$ & 199.29 \\
Li I & $1 s^{2} 8 s^{2} S-\left(1 s 2 p^{1} P\right) 8 s^{2} P$ & 199.26 \\
Li I & $1 s^{2} 9 s^{2} S-\left(1 s 2 p^{1} P\right) 9 s^{2} P$ & 199.25 \\
Li I & $1 s^{2} 10 s^{2} S-\left(1 s 2 p^{1} P\right) 10 s^{2} P$ & 199.23 \\
Li I & $1 s^{2} 11 s^{2} S-\left(1 s 2 p^{1} P\right) 11 s^{2} P$ & 199.24 \\
Li II & $1 s^{2} 1 S-1 s 2 p^{1} P$ \\
\end{tabular}

The autoionizing series observed by Ederer et al. in Li i photoabsorption spectra are shown in Figure 20.

As Table VI shows, and as is intuitively obvious, the lithium-like satellite lines with the second excited electron in higher $n$ levels merge with the resonance line of the helium-like ion. Thus, in calculating the flux in the resonance line, we should include the flux for those satellite lines which cannot be resolved from the resonance line. In the corona we should expect to see a second series corresponding to the transitions

LITHIUM VAPOR ABSORPTION

OF SYNCHROTRON RADIATION

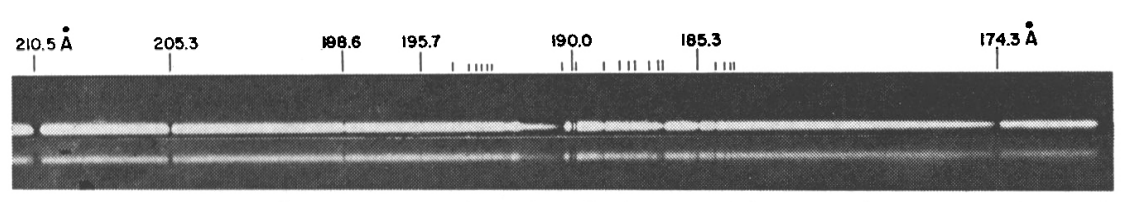

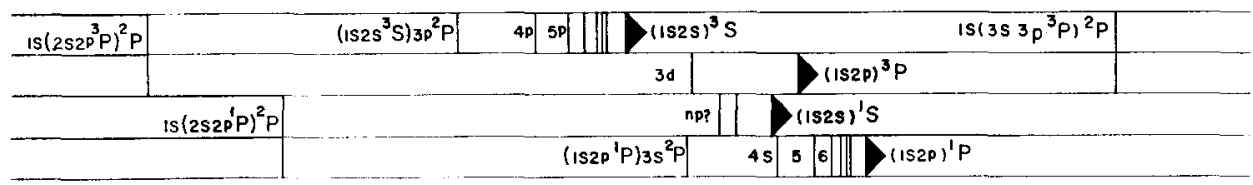

Fig. 20. Photo-absorption spectra of lithium showing autoionizing line series (Ederer et al., 1970). 


$$
1 s^{2} 2 p^{2} P-\left(1 s 2 p^{1} P\right) n p^{2} D,
$$

and transitions from other autoionizing series may also be observable. States such as $\left(1 s 2 s^{3} S\right) 3 p^{2} P$ and $\left(1 s 2 p^{1} P\right) 3 p^{2} D$ may decay by the transition of the $3 p$ electron, and will result in satellites to the $1 s^{2}{ }^{1} S-1 s 3 p^{1} P$ lines of helium-like ions. Walker and Rugge (1971) have observed these satellites in $\mathrm{MgX}$ and SixII, and have identified lines from doubly excited MgXI which appear as satellites to the Lyman- $\alpha$ line of MgXII. Figure 16 shows the position of a number of the autoionizing satellite lines which have been observed in the corona. It should be pointed out that the decay of the autoionizing state will generally result in a singly excited level, which will then decay. ${ }^{*}$ Thus, the decay of the MgXI $2 s 2 p^{3} P$ level is followed by the decay of the $1 s 2 s^{3} S$ level, and that of the $2 p^{2}{ }^{1} \mathrm{D}$ level by the decay of the $1 s 2 p^{1} P$ level. Doschek and Meekins (1971) have discussed this point. Sufficiently high electron density can decrease the effective dielectronic recombination rate due to the 'ionization' process

$$
\mathrm{e}+X^{+(z-1)}(i, n l) \quad X^{+z}(i)+\mathrm{e}+\mathrm{e},
$$

(Burgess and Summers, 1969, discuss this process in detail) where $X^{+(z-1)}(i, n l)$ represents a stabilized ion which has undergone dielectronic recombination but has not had time to allow the recombined electron to decay to the lowest unoccupied level. It should be emphasized that this process, although it decreases the recombination rate, will not decrease the rate of radiation due to stabilizing transitions, which are in general dipole transitions with lifetimes of $10^{-12} \mathrm{~s}$ or less.

Shore (1969) has given a rather complete discussion of dielectronic recombination ** and has derived formulas for dielectronic recombination rates. The flux at the Earth due to the capture of an electron, $\varepsilon$, from an initial state, $i$, into an autoionizing state, $d$, followed by the radiative decay of the state, $d$, into a stable state, $s$, is given by

$$
\frac{\mathrm{d}^{2} \phi_{\mathrm{D}}}{\mathrm{d} t \mathrm{~d} V}=n_{\mathrm{e}} n_{\mathrm{H}} A_{z} \frac{n_{i}}{n_{z}} 3.58 \times 10^{-28} \alpha^{\mathrm{di}}(i, \varepsilon \rightarrow d \rightarrow s) E_{d \rightarrow s} \operatorname{erg~cm}^{-2} \mathrm{~s} .
$$

The dielectronic recombination rate $\alpha^{\mathrm{di}}(i, \varepsilon \rightarrow d \rightarrow s)$ is given by

$$
\begin{aligned}
\alpha^{\mathrm{di}}(i, \varepsilon \rightarrow d \rightarrow s)= & 17.12 \frac{\exp (-\varepsilon / k T)}{T^{3 / 2}} \frac{\omega_{d}}{\omega_{i} \omega_{\varepsilon}} \times \\
& \times \frac{A^{\mathrm{rad}}(d \rightarrow s) A^{\text {auto }}(d \rightarrow i \varepsilon)}{\Gamma^{\mathrm{rad}}(d)+\Gamma^{\text {auto }}(d)}, \quad \mathrm{cm}^{-3} \mathrm{~s}^{-1}(\mathrm{~K})^{3 / 2},
\end{aligned}
$$

where $\varepsilon$ is the electron energy before capture, $\omega_{d}, \omega_{i}$ and $\omega_{\varepsilon}$ are statistical weights, $A^{\mathrm{rad}}$ and $A^{\text {auto }}$ are the transition probabilities of the state, $d$, for radiative decay to state, $s$, and autoionization to state $i, \varepsilon$, and $\Gamma^{\mathrm{rad}}$ and $\Gamma^{\mathrm{auto}}$ are the total widths for all modes of radiative decay or autoionization. Equation (4) may be used to compute the flux in a

* Note added in proof: Gabriel (1972) has given a complete account of the intensity and wave length of the $1 s^{2} n l-1 s 2 l 2 l^{\prime}$ satellite lines.

** Other treatments are to be found in the original paper by Burgess $(1964,1965)$ and in papers by Beigman et al. (1968) and by Trefftz $(1967,1969,1970)$. 
particular satellite line, or summed over the appropriate states $d$, the contribution of dielectronic recombinations to resonance line fluxes. There are calculations of $A^{\text {rad }}$ and $A^{\text {auto }}$ for some doubly excited helium-like states (references are given by Walker and Rugge, 1971 b); however, with the exception of several autoionizing widths for lithium (Cooper et al., 1970) there are few calculations available for lithium-like ions.

Burgess (1965) and Shore (1969) have assumed that the total dielectronic recombination rate is due to the capture of electrons into states of high values of $n$ and $l$, and have derived approximate rates based on this assumption. Shore's more accurate calculations agree with Burgess' rates for Li through $\mathrm{Ne}$ ions, but are lower for hydrogen and helium-like ions where the excited electron changes principal quantum numbers. I have extrapolated Shore's results (which extend only up to O vill) for hydrogen-like ions to SixIV, and renormalized them for helium-like ions. Using Shores's notation the total rate is given by

$$
\alpha(i, \text { tot })=\frac{\exp \left(-1.44 z^{2} / T\right)}{T^{3 / 2}} \alpha_{0} \frac{f_{1 s^{2}-1 s 2 p}^{\mathrm{Si} \text { XII }}}{f_{1 s-2 p}^{\mathrm{Si} \text { XIV }}} \frac{\lambda_{1 s-2 p}^{2}}{\lambda_{1 s^{2}-1 s 2 p}^{2}}
$$

with $\alpha_{0}$ set equal to $5.9 \times 10^{-2}$.

The resultant values of $\mathrm{d}^{2} \phi_{\mathrm{D}} / \mathrm{d} t \mathrm{~d} V$, and $\mathrm{d}^{2} \phi_{\mathrm{ex}} / \mathrm{d} t \mathrm{~d} V$ from Equation (2) are plotted in Figure 21. For temperatures between $5-7 \times 10^{6} \mathrm{~K}$, in which the SixIII resonance

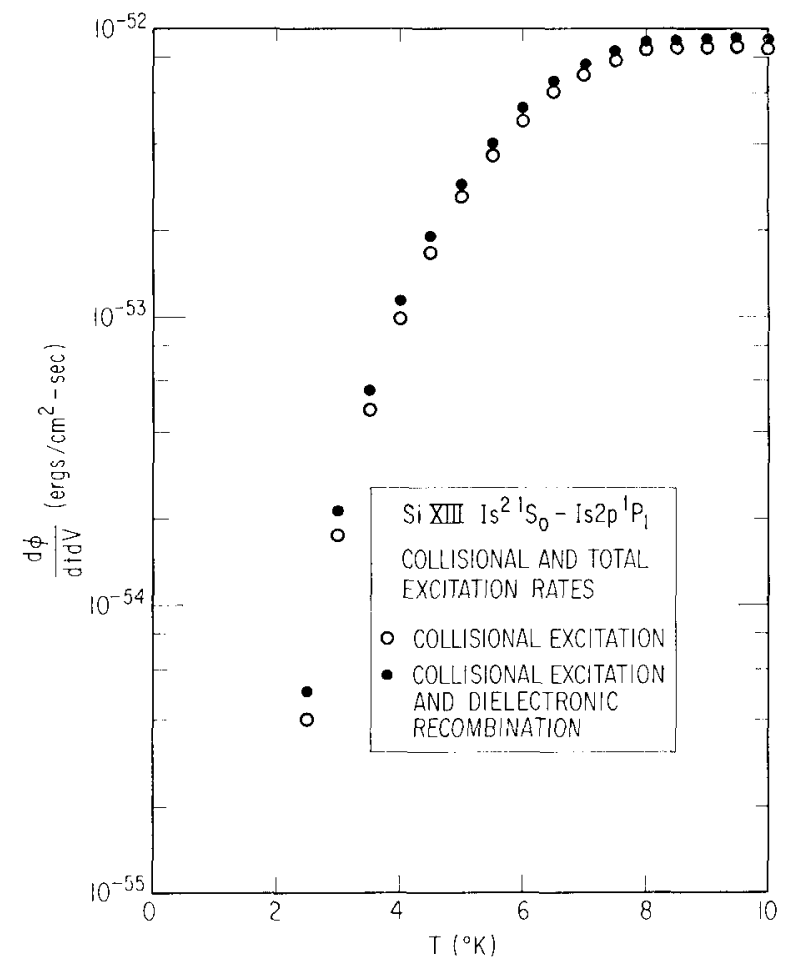

Fig. 21. Excitation function of the SIXIII resonance line, showing the importance of dielectronic recombination. 
lines are probably excited in the quiet corona, the dielectronic recombination rate into a single state (Walker and Rugge, 1971b, have measured the flux in the SiXII transition $1 s^{2} 2 s^{2} S-1 s 2 s 2 p^{2} P$ to be $16 \%$ of that in the SiXIII resonance line) exceeds the rate predicted by Figure 21. It would appear that the approximate treatments which assume that the total dielectronic recombination rate is due to capture into autoionizing states with high values of $n$ and $l$ must be reexamined, and more accurate autoionizing and radiative widths must be calculated for low lying levels.

In our discussion we have assumed that the total population of a particular stage of ionization resides in the ground state, and that all excitation processes start with the ion in the ground state. In some cases, however, the ground state is the lowest level within a doublet or triplet, or there is a low lying metastable excited state. In these situations the relative population of these levels is determined by the balance between collisional and radiation processes between these levels. Thus, the relative intensity of spectral lines which are preferentially excited from one of these low lying levels can depend on the properties of the coronal plasma. An example of the first situation is provided by boronlike ions with a ground term $2 s^{2} 2 p^{2} P_{1 / 2}$ and a low lying ${ }^{2} P_{3 / 2}$ term, and carbon-like ions with a $2 s^{2} 2 p^{2}{ }^{3} P_{0}$ ground term and low lying ${ }^{3} P_{2,1}$ levels. Widing (1966) discusses these levels for the coronal ions Si IX and Six. A similar situation prevails in the case of Fe vIII (KI sequence), Fex (Cli), Fexı(SI), FexIII(SiI) and FexIV (A1I). Pottasch (1966) has considered the excitation of these ions, and given level diagrams for them. Jordan (1966) and Petrini (1969) have also considered the excitation of Fexiv.

The case of an ion with a low lying excited configuration is illustrated by the beryllium-like ions, with the ground level $2 s^{2}{ }^{1} S$ and the metastable $2 s 2 p^{3} P_{2,1,0}$ level. A second example is the phosphorus-like ion FexII which has the ground level $3 s^{2} 3 p^{3}{ }^{4} S$, and the excited metastable level $3 s^{2} 3 p^{2} 3 d^{2} D_{5 / 2,3 / 2}$. The excitation of beryllium-like ions has been discussed by Jordan (1971) and by Munro et al. (1971). Pottasch (1966) has discussed the excitation of FexII.

For the ions which have low lying levels, it is of course necessary to determine the relative population of these levels before the population of higher levels can be computed (see for example Pottasch, 1966). In some cases these relative populations, and consequently the relative intensity of lines excited from these levels, are strongly dependent on electron density, and provide a spectroscopic method for the determination of density. We shall discuss these techniques in a later section.

\subsection{The DE-eXCitation OF EXCITED STATES}

Early theoretical studies of the coronal XUV spectrum concentrated on the analysis of resonance lines for which the radiated line flux is, to a good approximation, equal to the collisional excitation rate. For permitted lines from higher levels, we may modify the simple excitation rate by a suitable branching ratio, as has been discussed by Tucker and Koran (1971). Transition probabilities or oscillator strengths required to compute branching ratios are tabulated in the standard reference works by Wiese and his collaborators at the Bureau of Standards (Wiese et al., 1966, 1969; Smith and 
Wiese, 1971). A recent review by Layzer and Garstang (1968) and the text by Shore and Menzel (1968) discuss the computation of atomic transition probabilities in depth and give a complete list of references.

For metastable states, the decay path of a particular level cannot be predicted without a detailed knowledge of the transition probabilities from that level. Forbidden transitions from levels which are not metastable can also become important in the case highly charged ions and can compete effectively with allowed transitions in some cases. The calculation of transition probabilities for forbidden lines has been reviewed by Layzer and Garstang (1968), and transitions of astrophysical interest have been discussed by Garstang $(1969 a, b, c)$. The case of each forbidden transition must be treated individually, and in many cases the relative importance of a particular decay mode in an isoelectronic sequence is a strong function of nuclear charge.

An example of the decay of metastable levels is provided by the first excited states of helium-like ions. Dalgarno (1971) has discussed the transition probabilities for the ${ }^{1} P_{1}, 2^{1} S_{0}, 2^{3} P_{2,1,0}$ and $2^{3} S_{1}$ levels in helium-like ions. These transitions illustrate the complexity of the decay modes of metastable levels, the competition of forbidden decays and allowed decays for highly charged ions, and the importance of two-photon decay of metastable levels with total angular momentum $J=0$. Figure 22, taken from Dalgarno's paper illustrates the possible modes of decay for a helium-like ion, with the dominant mode $(s)$ for each level bracketed. We shall discuss the two-photon decay of the $2^{1} S_{0}$ level (and of the $2^{2} S_{1 / 2}$ level in hydrogenic ions) in the section on the X-ray continuum. The de-excitation of the $2^{3} P_{2}$ level provides an example of the competition between an allowed decay and a 'forbidden' decay. For low values of $z$, the allowed transition ${ }^{3} P_{2}-2^{3} S_{1}$ is the main decay mode. However, the magnetic quadrupole transition $2^{3} P_{2}-1^{2} S_{0}$ becomes increasingly important and for ArXVII its transition proba-

ENERGY LEVELS AND DECAY PATHS OF HELIUM

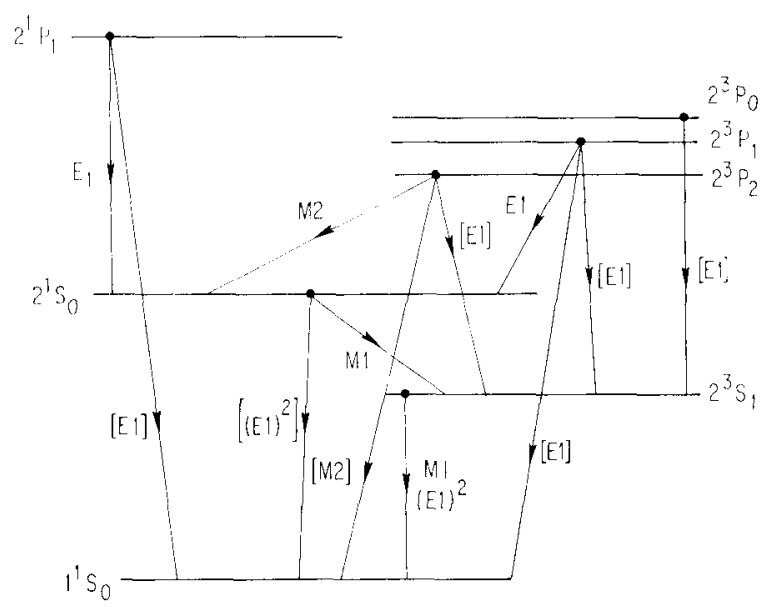

Fig. 22. Decay modes of the first excited states of the helium atom (after Dalgano, 1971). 
bility exceeds that of $2^{3} P_{2}-2^{3} S_{1}$ (Dalgarno, 1971). The identification of the singlephoton decay of the $2^{3} S_{1}$ level in the coronal spectrum by Gabriel and Jordan (1969a), and the theoretical verification by Griem (1969) that the single-photon decay mode of the $2^{3} S_{1}$ state is the dominant radiative decay mode, made possible the subsequent exploitation of the competitive collisional transfer of this level to the $2{ }^{3} P$ level as a method of determining coronal densities (Gabriel and Jordan, 1969b; Freeman et al., 1971). Freeman et al. and Blumenthal et al. (1971) have discussed the relative intensities of the $1 s^{2}{ }^{1} S_{0}-1 s 2 p^{3} P_{1}, 1 s^{2}{ }^{1} S_{0}-1 s 2 p^{3} P_{2}$, and $1 s^{2}{ }^{1} S_{0}-1 s 2 s^{3} S_{1}$ lines, and have demonstrated that the relative intensities of these lines are strongly dependent on nuclear charge. The latter authors make use of the Coulomb-Born collision strengths calculated by Burgess et al. (1970) to calculate directly $1 s^{21} S-1 s 2 p^{3} P$ and $1 s^{2}{ }^{1} S-1 s 2 s^{3} P$ excitation rates, and also calculate explicitly the effects of cascades and recombinations. Rugge and Walker (1971a,b) have measured the ratio of the ${ }^{3} S_{1}$ line to the ${ }^{3} P_{2}$ and ${ }^{3} P_{1}$ lines of $\mathrm{O}$ VII and have found a value of 3.78 , independent of solar activity. This result is in good agreement with the theoretical value of 3.85 expected in the absence of collisional de-excitation of the metastable ${ }^{3} S$ level. A second example of the competitive de-excitation of an excited level by allowed and forbidden transitions is provided by the $3^{3} P_{1}$ level which can decay via $3^{3} P_{1}-2^{3} S_{1}$ Figure 16 a shows a portion of the solar spectrum obtained near the time of a $1 \mathrm{~B}$ flare in which the spin forbidden transition $3{ }^{3} P_{1}-1{ }^{1} S_{0}$ is resolved from the $3^{1} P_{1}-1{ }^{1} S_{0}$ line for MgXI (Walker and Rugge, $1971 \mathrm{~b})$.

The identification of lines corresponding to the decay of metastable levels in the helium-like ion has stimulated the observation of a number of these processes in the laboratory. Marrus and Schmieder (1970a) have measured the lifetime of the $2^{3} S_{1}$ state of ArXVII and have also observed the magnetic quadrupole decay of the $2^{3} P_{2}$ state of ArXVII (1970b). Elton at al. (1968) have observed the two-photon decay of the $2^{1} S_{0}$ level of NeIX, and Schmieder and Marrus (1970) have measured the lifetime of the $2^{2} S_{1 / 2}$ state of ArXVIII for two-photon decay.

The de-excitation of the $2 p^{5} 3 \mathrm{~d}, 2 p^{5} 3 p$ and $2 p^{5} 3 s$ levels in neon-like ions such as FexvII provides an additional example of the importance of a detailed knowledge of transition probabilities to the interpretation of the coronal spectrum. Garstang (1966) has computed the transition probabilities for transitions within these levels and to the ground state. We have already discussed the dominant role of cascades from the $2 p^{5} 3 p$ level in populating the $2 p^{5} 3 s$ level of Fexvir in the corona.

Garstang (1969a) has discussed the role of magnetic quadrupole radiation in deexciting metastable levels in the corona and has tabulated the wavelengths and transition probabilities and given references to original work for a number of important lines. An interesting example is the $2 p^{5} 3 s^{3} P_{2}$ level in Fexvil which is the lowest excited state of FexvII and is consequently metastable. Walker and Rugge (1971c) have identified this transition in the solar spectrum (see Figure 15).

The de-excitation of doubly excited levels is also of importance in the solar corona, in view of the strong satellite lines observed from autoionizing levels. The excitation of these lines has been discussed in the previous section. There are as yet very few theore- 
tical calculations of the radiative and autoionizing lifetimes of these states (see Walker and Rugge, $1971 \mathrm{~b}$ for a review of theoretical calculations on these levels).

\subsection{RECOMBINATION PROCESS AND THE X-RAY CONTINUUM}

There are three processes which contribute to the X-ray continuum; thermal bremsstrahlung, radiative recombination, and two-photon decay of metastable ${ }^{2} S$ and ${ }^{1} S$ states of helium-like and hydrogen-like ions. For the temperatures generally found in the quiet corona radiative recombination is the most important process, while for the temperatures attained in flares thermal bremsstrahlung becomes the dominant continuum process.

Culhane (1969) has presented the most complete discussion of the two 'classical' continuum processes, thermal bremsstrahlung and radiative recombination, including an explicit sum with the appropriate Gaunt factors for elements with abundances greater than $4 \times 10^{-6}$ (relative to hydrogen) for both processes.

Following Culhane, we may write the bremsstrahlung flux at the earth generated per unit volume in the corona as

$$
\begin{aligned}
& \frac{\mathrm{d}^{3} \phi}{\mathrm{d} t \mathrm{~d} V \overline{\mathrm{d} \lambda}=\frac{7.15 \times 10^{-47}}{\sqrt{T \lambda^{2}}}} \exp (-h c / \lambda k T) n_{\mathrm{e}} n_{\mathrm{H}} \times \\
& \times\left\{\sum_{z}\left[A_{z} z_{i}^{2} \bar{g}\left(z_{i}, T, c / \lambda\right)\right]\right\} \mathrm{erg} \mathrm{cm}^{-2} \mathrm{~s} \AA,
\end{aligned}
$$

where $T$ is the coronal temperature in $\mathrm{K}, \lambda$ is the photon wavelength in Ångströms, $n_{\mathrm{e}}$ and $n_{\mathrm{H}}$ are the electron and hydrogen ion densities, $A_{z}$ is the abundance relative to hydrogen, of element $z, z_{\mathrm{i}}$ is the effective charge of element $z$, and $\bar{g}\left(z_{\mathrm{i}}, T, c / \lambda\right)$ is the temperature-averaged free-free hydrogenic Gaunt factor. Karzas and Latter (1961) have computed and tabulated the temperature-averaged Gaunt factors.

For radiative recombination, the flux at the Earth due to the recombination to level $n$ of element $z$ in ionization state $i+1$ is given by

$$
\begin{aligned}
& \mathrm{d} t \mathrm{~d} V \mathrm{~d} \lambda=\frac{12.02 \times 10^{-44}}{T^{3 / 2} \lambda^{2}} n_{\mathrm{e}} n_{\mathrm{H}} A_{z}{ }^{n_{i+1}} X_{z, i, n}^{2} n \frac{\xi_{n}}{2 n^{2}} \times \\
& \quad \times\left\{\bar{g}\left(z_{i+1}, n, c / \lambda\right) \exp -\left(h c / \lambda-X_{z, i, n}\right) / k T\right\} \operatorname{erg~cm}{ }^{-2} \mathrm{~s} \AA,
\end{aligned}
$$

where $X_{z, i, n}$ is the ionization potential (in electron volts) from level $n$ of element $z$ ionization state $i, n_{i+1} / n_{z}$ is the fractional population and $z_{i+1}$ the effective charge of element $z$ in ionization state $i+1$, and $\bar{g}\left(z_{i+1 /} n, c / \lambda\right)$ is the hydrogenic recombination gaunt factor averaged over $l$ states for each level. The quantity $\xi_{n} / 2 n^{2}$ is the unoccupied fraction of the $n$ shell in the ion $i+1$, and Equation (6) is valid for wavelengths $\lambda \leqslant h c / X_{z, i, n}$. The other symbols have the same meaning as before. The recombination gaunt factors required have also been calculated and tabulated by Karzas and Latter.

As we pointed out earlier, radiative recombination into an excited level contributes to the population of that level. The rate for that process can be calculated from Equation (5), with the appropriate values of $n$, and with $\left(\xi_{n} / 2 n^{2}\right) \equiv 1$. The specific 
value of $g$ for each angular momentum sublevel, $g\left(z_{i+1}, n, l, c / \lambda\right)$ should be used, and Equation (6) integrated over wavelength to find the total recombination rate for the level in question.

Smith (1969) and Dalgarno and Drake (1969) have pointed out that the $2 s^{2} S$ and $1 s 2 s^{1} S$ metastable levels of hydrogen-like and helium-like ions in the solar corona should de-excite by two-photon decay. The resultant continuum should make substantial contributions to the X-ray continuum, especially between 20 and $50 \AA$. Elton et al. (1968) have observed the two-photon continuum of Ne IX in a laboratory plasma. Dalgarno (1969) has reviewed the calculation of the transition rates and spectral distribution of the two-photon decay of hydrogen and helium.

The spectral distribution of the two-photon continuum of hydrogen-like ions has been calculated by Spitzer and Greenstein (1951). For helium-like ions, the shape of the two-photon continuum is dependent on nuclear charge. Dalgarno and Drake (1969) have computed transition rates and the spectra shape of the two-photon decay of the ${ }^{1} S$ state for ions up to NeIX.

We shall compute the excitation rates according to the formula

$$
\begin{aligned}
& \frac{\mathrm{d}^{2} \phi}{\mathrm{d} t \mathrm{~d} V}=n_{\mathrm{e}} n_{\mathrm{H}} A_{z} \frac{n_{i}}{n_{z}} 5.01 \times 10^{-44} \bar{z}^{2} \Omega_{1 s-2 s} \\
& \times\left\{\frac{\exp \left(-E_{1 s-2 p} / k T\right)}{\sqrt{ } T}\right\} \mathrm{erg} \mathrm{cm}^{-2} \mathrm{~s}
\end{aligned}
$$

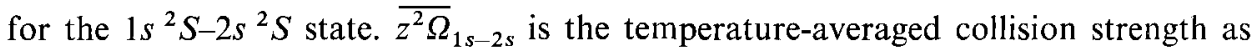
calculated by Burgess et al., and is tabulated in Table IV. The coronal two-photon flux from a hydrogen-like ion is then

$$
\begin{aligned}
\frac{\mathrm{d}^{3} \phi_{2 \gamma}}{\mathrm{d} t \mathrm{~d} V} \frac{\mathrm{d} \lambda}{} & =n_{\mathrm{e}} n_{\mathrm{H}} A_{z} \frac{n_{i}}{n_{z}} \phi\left(\frac{\lambda_{1 s-2 s}}{\lambda}\right) \frac{\lambda_{1 s-2 s}}{\lambda^{2}} 5.01 \times 10^{-44} \times \\
\times\left\{\bar{z}^{2} \Omega_{1 s-2 s}\left(3 k T / 4 E_{1 s-2 s}\right)\right. & \left.\frac{\exp \left(-E_{1 s-2 s} / k T\right)}{\sqrt{ } T}\right\} \mathrm{erg} \mathrm{cm}^{-2} \mathrm{~s} .
\end{aligned}
$$

The function $\phi$ is the two-photon decay spectral distribution calculated by Spitzer and Greenstein, and is given in terms of $y$, the fractional energy carried off by one photon.

$$
\phi(y)=\phi\left(\frac{E}{E_{1 s-2 s}}\right)=\phi\left(\frac{\lambda_{1 s-2 s}}{\lambda}\right) .
$$

The function $\phi$ is symmetric about $y=0.5$, and is normalized so that

$$
\int_{0}^{1} \phi(y) \mathrm{d} y=2 .
$$

The wavelength $\lambda=\lambda_{1 s-2 s}$ corresponds to one photon taking all of the energy in the decay. For helium-like ions we have multiplied Equation (7) by the ratio of the oscil- 
lator strengths

$$
f_{\left(1^{1} S-21 P\right)} / f_{\left(1^{2} S-2^{2} P\right)}
$$

of the helium-like and hydrogen-like resonance transitions, and used the NeIx spectral distribution calculated by Dalgarno and Drake (1969) for ions heavier than Ne Ix. The spectral distribution is a very slowly varying function of $z$.

In a later section we shall use the formulas developed in this section to discuss the relative importance of $\mathrm{X}$-ray production processes in the corona.

\subsection{TECHNIQUeS FOR THE DIRECT MEASUREMENT OF PHYSICAL PARAMETERS}

An important result of the study of the line spectrum of the corona is the development of techniques which allow the direct determination of the physical properties of the coronal plasma, without a detailed model of coronal structure. The methods we will discuss involve the measurement of line intensity ratios which are sensitive to a particular parameter, such as electron temperature, density, or relative abundance, but are insensitive to the detailed structure of the corona.

There are three techniques which have been proposed for the determination of coronal temperatures. They generally involve the intensity ratio of lines which depend on the population of only one ionization stage of a single ionic species, but which have excitation functions which depend on temperature in different ways.

Heroux (1964) has proposed a method which involves the ratio of the $2 s-2 p$ and $2 s-3 p$ line intensities of lithium-like ions. These lines differ in excitation energy by an order of magnitude. This line ratio is given, in the Bethe approximation, by Heroux as

$$
I_{2 p}=\frac{I\left(2 s^{2} S_{1 / 2}-2 p^{2} P_{3 / 2}\right)}{I\left(2 s^{2} S_{1 / 2}-3 p^{2} P_{3 / 2,1 / 2}\right)}=\frac{f_{2 p} X_{2 p} \bar{g}\left(X_{2 p} / k T\right)}{f_{3 p} X_{3 p} \bar{g}\left(X_{3 p} / k T\right)}\left\{\exp \left[\begin{array}{c}
X_{2 p}-X_{3 p} \\
k T
\end{array}\right]\right\},
$$

where $f$ is the oscillator strength and $X$ the excitation energy of the transition, and $\bar{g}$ is the Kramers-Gaunt function (Allen, 1963). Since $g$ depends only weakly on temperature and $X_{2 p} / k T<1, I_{2 p} / I_{3 p} \sim \exp \left(-X_{3 p} / k T\right)$, which is a sensitive function of temperature. Figure 23 shows the ratio $I_{2 p} / I_{3 p}$ as calcualted by Heroux for the abundant coronal ions up to SixII. The line intensity ratio $I_{2 p} / I_{3 p}$ for lithium-like ions should provide a sensitive technique for the determination of coronal temperatures up to $2 \times 10^{6} \mathrm{~K}$ if the lines of SxIV (which have not yet been identified in the coronal spectrum) can be used in addition to those of the ions included in Figure 23. One drawback of this method is the difficulty of maintaining accurate calibrations in a satellite or rocket spectrometer over the broad wavelength range which separates the $2 s-2 p$ and $2 s-3 p$ transitions. Flower (1972) has recently applied this method to the lines of OVI, Ne vilI, and $\mathrm{Mgx}$.

A second type of line intensity ratio which is temperature sensitive is that for a resonance transition and a higher transition in the same series. The case which is the most tractable theoretically is the Lyrian- $\alpha /$ Lyman- $\beta$ intensity ratio for hydrogen-like ions. McWhirter and Hearn (1963) provided the first analysis of these line intensity 


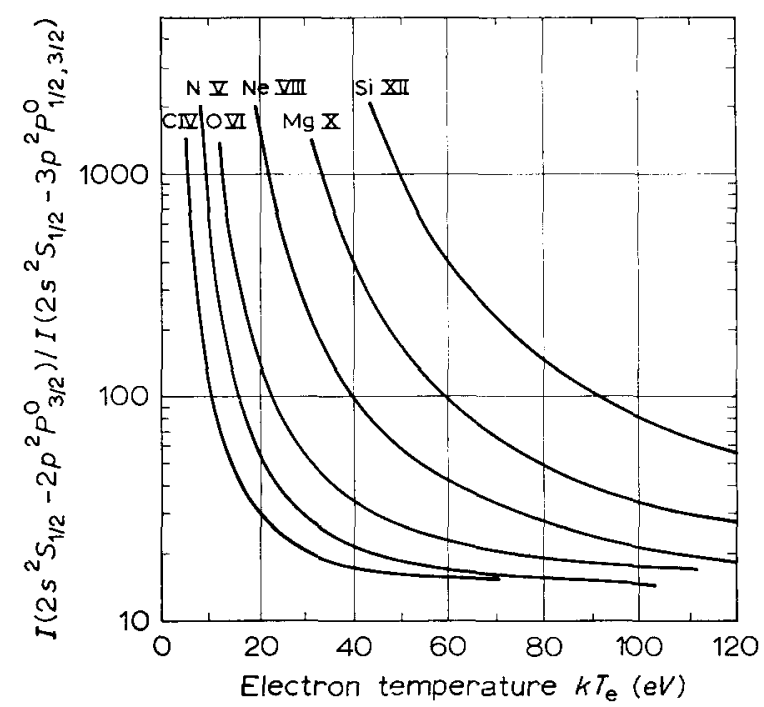

Fig. 23. The theoretical spectral line intensity ratio of the transitions $2 s^{2} S_{1 / 2}-2 p^{2} P_{3 / 2}$ and $2 s^{2} S_{1 / 2-}$ $-3 p^{2} P_{1 / 2,3 / 2}$ against $k T_{\mathrm{e}}$ for several ions of the Li-like isoelectronic sequence (Heroux, 1964).

ratios, and Beigman and Vainsthein (1967) and Jacob (1968) have performed more detailed calculations for the case of O viII. A number of authors (Blake et al., 1965; Evans and Pounds, 1968) have used this technique to determine coronal temperatures based on the $\mathrm{O}$ VIII line intensity ratios. However, in the case of $\mathrm{O}$ VIII this technique must be applied with caution, because the FexviII $2 p^{5}{ }^{2} P_{3 / 2}-2 p^{4}\left({ }^{3} P\right) 3 s^{4} P_{3 / 2}$ transition at $16.01 \AA$ is blended with the $O$ vIII Lyman- $\beta$ line at $16.006 \AA$ (Walker and Rugge, 1969). The FexvII line is important only when there are hot active regions present, and its presence can be detected by observing the FexviI $2 p^{52} P_{3 / 2}-2 p^{4}\left({ }^{3} P\right) 3 s^{2} P_{3 / 2}$ transition, which is observed to be of comparable intensity in laboratory spark spectra (Cohen et al., 1968).

Gabriel (1972) has pointed out that the excitation function of the dielectronic recombination satellite lines, which have been observed in coronal spectra, has a different temperature dependence than the excitation function for collisionally excited resonance lines. From Equation (1), we may write the flux in a resonance line $(R)$ as

$$
I_{R} \propto \overline{z^{2} \Omega(T)} \exp \left(-E_{R} / k T\right) / \sqrt{T} .
$$

For a satellite line $(S)$ we can write, from Equation (4),

$$
I_{S} \propto \frac{A^{\text {rad }} A^{\text {auto }}}{\Gamma^{\text {rad }}+\Gamma^{\text {auto }}} \exp \left(-E_{S} / k T\right) / T^{3 / 2} .
$$

The collision strength $\overline{z^{2} \Omega}(T)$ is a slowly varying function of temperature. Provided that we can compute the transition probabilities $A^{\text {rad }}$ and $A^{\text {auto }}$, and the level widths $\Gamma^{\mathrm{rad}}$ and $\Gamma^{\text {auto }}$, we can express the relative intensities of the lines in terms of the known 
transition energies and temperature alone. For ions of sufficiently low nuclear charge, the autoionizing widths will greatly exceed the radiative width. Assuming that there is only one probable decay mode (i.e. $\Gamma=A$ ) and recognizing the strong dependence of $A^{\text {rad }}$ on $z$, we can write

$$
I_{S} / I_{R} \propto A^{\mathrm{rad}}(z)\left\{\exp \left[\left(E_{R}-E_{\mathrm{S}}\right) / k T\right]\right\} / T .
$$

Grineva et al. (1971) have used this technique to determine the temperature of a flaring region, using the Fexxv resonance line, and a FexxIV satellite line.

Techniques have been evolved for measuring coronal densities which involve the dependence of line intensity ratios on the population of metastable levels. For electron densities in the range such that collisional and radiative de-excitation rates for these levels are competitive, the level population and consequently the line intensity ratios become sensitive to density.

An example is the ratio $(R)$ of the intensity of the $2{ }^{3} S_{1}-1{ }^{1} S_{0}$ line from the $2{ }^{3} S_{1}$ metastable level to that of the $2{ }^{3} P_{2}-1{ }^{1} S_{0}$ and $2{ }^{3} P_{1}-1{ }^{1} S_{0}$ lines in helium-like ions. This line ratio has already been discussed in the section on line de-excitation, for the case of low densities, when collisional de-excitation of $2{ }^{3} S_{1}$ is unimportant. As Gabriel and Jordan (1969b) point out in their original paper (see also Freeman et al., 1971; and Blumenthal et al., 1971), there is a range of densities for which $R$ becomes sensitive to density; and this density range occurs at higher and higher values as nuclear charge, and consequently the transition rate for $2{ }^{3} S_{1}-1{ }^{1} S_{0}$, increases. For normal coronal densities $\left(\sim 10^{8} \mathrm{~cm}^{-3}\right)$ only the $\mathrm{CV}$ line ratio should be sensitive, and this sensitivity is complicated by the excitation $2{ }^{3} S-2{ }^{3} P$ caused by the coronal UV flux. A number of authors (Walker and Rugge, 1970; Batstone et al., 1970; Freeman et al., 1971) have used this technique to evaluate coronal densities. These early results suggested very high coronal densities. These results must be reevaluated in view of the realization of the importance of the lithium-like satellite lines, which can contribute significantly to the flux in both the $2^{3} S \rightarrow 1{ }^{1} S$ and $2{ }^{3} P \rightarrow{ }^{1} S$ lines (Walker and Rugge, 1971b).

More recently, new results have suggested that, at least for quiet (i.e. non-flare) conditions the value of $R$ for OVII does not vary, implying that $n_{\mathrm{e}}<5 \times 10^{9} \mathrm{~cm}^{-3}$. Rugge and Walker (1971a,b) have shown that $R$ is independent of the total level of solar activity, and Acton et al. (1971) have demonstrated that $R$ does not vary across the solar disk by using a collimated crystal spectrometer which scanned across the disk. In both cases, the measured value of $R$ was in good agreement with the theoretical value of 3.6-3.8 in the low density limit.

A second technique for density determination is based on the relative intensity of lines which are preferentially excited from excited levels within the ground configuration or a low lying configuration. Jordan (1971) and Munro et al. (1971) have analyzed the situation for beryllium-like ions. The ground terms is $2 s^{2}{ }^{1} S$, however for sufficiently high densities the metastable $2 s 2 p^{3} P_{2,1,0}$ levels have a substantial population. The $2 p^{2}{ }^{3} P$ level can only be excited from this level, so that the ratio of the $2 s 2 p^{3} P-2 p^{2}{ }^{3} P$ line to any line which can be excited from the ground term $\left(2 s^{2}{ }^{1} S-2 s 2{ }^{1} P\right.$ for exam- 
ple) is strongly density dependent. These authors have applied this technique to the observations obtained by the Harvard EUV spectroheliographs on OSO 4 and OSO 6. Widing (1966) has discussed a similar technique for the boron-like ion Sx.

The determination of the relative abundance of elements is perhaps the most difficult problem of coronal physics and places the most severe demands on the observational data. If, however, we can find two ions of different elements which have excitation functions with a very similar dependence on temperature, at least over the temperature ranges of importance in the corona, then we can determine relative abundances directly from the line ratios without a detailed knowledge of coronal structures. The resonance lines of $\mathrm{NeX}$ and MgXI satisfy this requirement quite closely, and for a range of coronal models which were fitted to observed line fluxes below $25 \AA$, demonstrated the independence of the $\mathrm{Mg} / \mathrm{Ne}$ abundance ratio from detailed characteristics of a particular model. The utility of this procedure will be more fully described in the section on coronal models.

\subsection{The Relative importance of Emission Processes in the CORONAL SPECTRUM}

Before we are able to use the formulas developed in the previous sections to calculate the relative importance of the various emission processes in forming the coronal spectrum, we must know the relative coronal abundances, $A_{z}$. A section on the problem of coronal abundances is included in the discussion of Section 4. The abundances which we have adopted are given in Table VII.

TABLE VII

\begin{tabular}{llll}
\hline Element & Abundance $\left(A_{z}\right)$ & Element & Abundance $\left(A_{z}\right)$ \\
& & $\mathrm{Mg}$ & $31 \times 10^{-6}$ \\
$\mathrm{H}$ & 1 & $\mathrm{Al}$ & $2 \times 10^{-6}$ \\
$\mathrm{He}$ & $0.07(0.16)^{\mathrm{a}}$ & $\mathrm{Si}$ & $31 \times 10^{-6}$ \\
$\mathrm{C}$ & $375 \times 10^{-6}$ & $\mathrm{~S}$ & $16.5 \times 10^{-6}$ \\
$\mathrm{~N}$ & $88 \times 10^{-6}$ & $\mathrm{~A}$ & $3 \times 10^{-6}$ \\
$\mathrm{O}$ & $300(440)^{\mathrm{a}} \times 10^{-6}$ & $\mathrm{Fe}$ & $31 \times 10^{-6}$ \\
$\mathrm{Ne}$ & $26 \times 10^{-6}$ & & \\
\hline
\end{tabular}

a The values adopted are those recommended by Withbroe (1971) except for $\mathrm{He}$ and $\mathrm{O}$, where Withbroe's recommended values are given in parenthesis.

A number of authors (Culhane, 1969; Landini and Monsignori Fossi, 1972; Tucker and Koran, 1971; Beigman et al., 1971; Mewe, 1972a,b) have given graphical and tabular results for the theoretical coronal spectrum.

In Figure 24 we present results for the spectrum below $25 \AA$ for two temperatures. We have included only resonance lines of $\mathrm{N}, \mathrm{O}, \mathrm{Ne}, \mathrm{Mg}, \mathrm{Al}, \mathrm{Si}, \mathrm{S}$, and Fexvil. The continuum includes the flux due to thermal bremsstrahlung, radiative recombination, and two-photon decay. We have not included forbidden lines, or permitted lines from levels above the first excited level, in order to simplify the graph. We also have not 


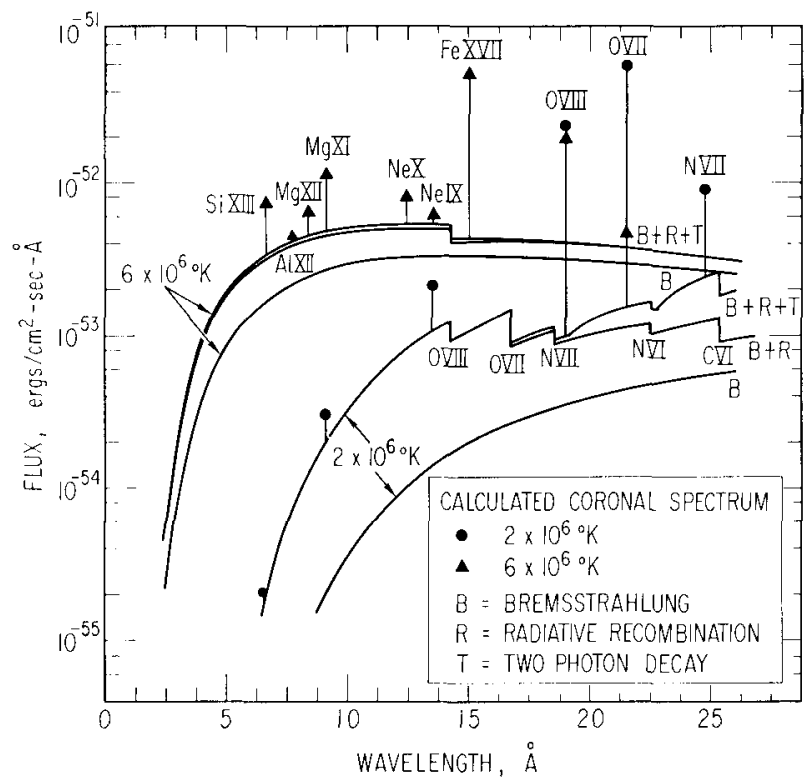

Fig. 24. Calculated coronal spectrum between 1 and $25 \AA$, including thermal bremsstrahlung, recombination radiation, two-photon decay, and the collisionally excited resonance lines of $\mathrm{N}, \mathrm{O}, \mathrm{Ne}$, $\mathrm{Mg}, \mathrm{A}, \mathrm{S}$, and Fe. The FexviII spectrum is not included (see text).

included satellite lines, or the contribution of dieletronic recombination to resonance line fluxes. Finally, we have not included the spectrum of FexvIII, which should be visible at $6 \times 10^{6} \mathrm{~K}$. The identification of FexvIII lines in the solar spectrum is not in a completely satisfactory state, and collision strengths for the individual line multiplets are not available. The problem of the FexvIII spectrum of the corona has been discussed in the Section 2.4 .

The spectrum at $2 \times 10^{6} \mathrm{~K}$ should be representative of the most probable temperature in the quiet corona, while that for $6 \times 10^{6} \mathrm{~K}$ should be representative of the highest temperatures in active regions. An interesting feature of Figure 24 is the important contribution of the two-photon decay of O VII $2^{1} S$ and O VIII $2^{2} S$ to the continuum beyond $20 \AA$. The decay of $\mathrm{CV} 2^{1} S$ and $\mathrm{CVI} 2^{2} S$ should make a similar contribution at longer wavelengths.

We shall make use of these spectral computations in discussing coronal models in Section 4.

\section{The Structure of the Quiet Corona}

It is difficult to rigidly classify studies of coronal structure based on the X-ray spectrum into investigations of elemental abundances or temperature structure, or density. Our division of the analysis of the coronal X-ray spectrum into sections on elemental abundances, temperature, and density structure will therefore be somewhat arbitrary; however, it will serve to focus attention on the three fundamental properties of the 
coronal plasma which we must determine. A fundamental difficulty inherent in the presently available analysis is their inability to distinguish individual active regions and the quiet corona. This problem, of course, reflects the lack of spatially resolved, spectral observations. Observations at XUV wavelengths made by the Harvard College Observatory OSO-4 and OSO-6 spectroheliographs, can observe the coronal resonance lines of the alkali-like ions of Mgx, SixII, and FexVI with 1' (OSO 4) and $35^{\prime \prime}$ (OSO 6) resolution spectively. The analysis of these observations, which have been reviewed by Noyes (1971), have indicated that the line flux enhancements observed for these lines can be explained by a 5-fold increase in active region density and only a modest increase in active region temperature. These lines are not sensitive to temperatures above $\sim 3.0 \times 10^{6} \mathrm{~K}$, and models based on X-ray spectra will alter this picture to some extent.

\subsection{Elemental abundances}

The relative abundance of elements in the Sun has been inferred by a number of techniques; including the analysis of permitted and forbidden photospheric absorption lines, the analysis of chromospheric emission lines, the analysis of coronal forbidden lines in the visible and of permitted lines in the XUV, the analysis of meteorites and the observation of solar cosmic rays, and of the solar wind.

The pioneering work of Pottasch (1967 and the references cited therein) in analyzing the EUV spectra obtained by Hinteregger and his colleagues at AFCRL provided a detailed temperature structure for the quiet corona, and a technique to determine relative coronal abundances. Pottasch was able to include lines from a sufficient number of ionization stages of iron and of silicon (and consequently a broad temperature range for each element) to define an atmospheric structure for each element. The results of this analysis forced an increase of an order of magnitude in the coronal abundance ratio $A_{\mathrm{Fe}} / A_{\mathrm{Si}}$, compared to the then currently accepted photospheric value. More recently, the relative abundance of ions in the solar wind has been measured directly (Bame et al., 1970; Holtzer and Axford, 1970; Lange and Scherb, 1970). These results strongly support the revised iron abundances.

The redetermination of FeI and FeII oscillator strengths has led to the revision of the photospheric iron abundance (Gartz et al., 1969a) so that it is consistent with coronal determinations of the Fe/Si ratio (Jordan and Pottasch, 1968) and with results from meteorites (Gartz et al., 1969b) and the solar wind (Bame et al., 1970). There is little solid evidence to support a systematic difference in coronal and photospheric abundances. Withbroe (1971) has reviewed the current status of solar abundances determined by various methods. The photospheric and coronal abundances adapted by Withbroe in his recent review are essentially the same, with the only substantial differences being in the oxygen abundances. We shall, as a starting point, adopt Withbroe's recommended XUV values with the exception of oxygen, where we shall use a value of $300 \times 10^{-6}$, which is more consistent with recent XUV analyses (Chambre, 1971) than Withbroe's value of $440 \times 10^{-6}$. The question of the helium abundance presents a more difficult problem. The XUV lines of a range of ionization stages of $\mathrm{C}, \mathrm{N}, \mathrm{O}$, 
$\mathrm{Ne}, \mathrm{Mg}$, Si and Fe may all be related in a single self-consistent analysis (for example Pottasch, 1967), so that the relative abundance of this group of elements may be determined from the same coronal volume with some confidence. The lines of helium, however, are formed at the base of the transition region, and no such self consistent analysis is possible (this problem arises also in determining absolute abundances relative to hydrogen). There are no direct determinations of solar photospheric helium abundances using spectroscopic methods. Lambert (1967) using the ratio $N_{\mathrm{He}} / N_{\mathrm{O}}=107$ as measured from cosmic ray analysis concluded that $A_{\mathrm{He}}=0.063$. Bame et al., for a particular set of solar wind observations, have measured abundance ratios $A_{\mathrm{He}}, A_{\mathrm{O}}$, $A_{\mathrm{Si}}, A_{\mathrm{Fe}}$ of $0.03,2 \times 10^{-4}, 4.2 \times 10^{-5}$, and $3.4 \times 10^{-5}$. Robbins et al. (1970) studied the long term variation of $N_{\mathrm{He}} / N_{\mathrm{H}}\left(=A_{\mathrm{He}}\right)$ in the solar wind and found a mean value $A_{\mathrm{He}} \sim 0.04$, with only $2 \%$ of the data having $A_{\mathrm{He}}>0.1$. Perhaps the result which is most pertinent to our problem is that of Pottasch, who found $N_{\mathrm{He}} / N_{\mathrm{Si}}=2000$. The relative helium abundance is of considerable importance in the analysis we shall present below, since we will attempt to normalize out relative abundances by considering the relative intensity of emission lines and of the adjacent continuum. If we were to adopt Withbroe's XUV abundance of $A_{\mathrm{He}}=0.16$, then roughly half of the bremsstrahlung continuum would be due to helium ions. We shall adopt a helium abundance $A_{\mathrm{He}}$ of 0.07 , which is consistent with the $A_{\mathrm{Si}}$ of $3.1 \times 10^{-5}$ recommended by Withbroe, and the Pottasch $N_{\mathrm{He}} / N_{\mathrm{Si}}$ determination, as well as Lambert's 'photospheric' abundance. The abundance we have adopted are shown in Table VII. The abundance recommended by Withbroe is given in parenthesis in those cases where we have chosen a different abundance. The coronal spectrum computed with these abundances has been discussed, and is presented in Figure 24.

In order to illustrate the problems of coronal abundance determination from X-ray spectra, we have used the absolute fluxes obtained from the OV 1-10 satellite for 4 January 1967 (Walker and Rugge, 1971a). The spectrum is shown in Figure 2, and is

TABLE VIII

Measured and calculated line fluxes for January 4, 1967

\begin{tabular}{|c|c|c|c|c|c|c|}
\hline \multirow[t]{2}{*}{ Ion } & \multirow[t]{2}{*}{ Transition } & \multirow{2}{*}{$\begin{array}{l}\text { Wavelength } \\
\AA\end{array}$} & \multirow[t]{2}{*}{ Exp } & \multicolumn{3}{|c|}{ Flux $\left(\operatorname{erg} \times 10^{5}\right)$} \\
\hline & & & & Model I & Model II & Model III \\
\hline O vir & $1 s^{2}{ }^{1} S-1 s 2 p^{1} P$ & 21.60 & 241.5 & 245.8 & 243.5 & 244.5 \\
\hline O VIII & $1 s^{2} S-2 p^{2} P$ & 18.97 & 267 & 250.3 & 248 & 271.7 \\
\hline FexviI & $2 p^{6}{ }^{1} S-2 p^{5} 3 d^{1} P$ & 15.01 & 113 & - & - & - \\
\hline NeIX & $1 s^{2}{ }^{1} S-1 s 2 p^{3} P$ & $13.55^{\mathrm{a}}$ & 35 & 31.6 & 32 & 24.3 \\
\hline NeIx & $1 s^{2}{ }^{1} S-1 s 2 p^{1} P$ & $13.45^{\mathrm{a}}$ & & & & \\
\hline $\mathrm{NeX}$ & $1 s^{2} S-2 p^{2} P$ & 12.13 & 10.5 & 10.4 & 10.5 & 10.7 \\
\hline Mgxi & $1 s^{2}{ }^{1} S-1 s 2 p^{3} P$ & $9.23^{\mathrm{a}}$ & 12 & 13 & 13.5 & 12.4 \\
\hline $\mathrm{MgXI}$ & $1 s^{21} S-1 s 2 p^{1} P$ & $9.17^{a}$ & 12 & 13 & 13.3 & 12.4 \\
\hline
\end{tabular}

a The ${ }^{3} P$ and ${ }^{1} P$ lines are not resolved in this spectrum. The values of $R=\left[I\left({ }^{3} S\right) /\left(I^{3} P\right)\right]$ computed by Freeman et al. (1970) and of $G=\left[I\left({ }^{3} S+{ }^{3} P\right) / I\left({ }^{1} P\right)\right]$ measured by Rugge and Walker (1971a) were used to compute $I\left({ }^{1} P+{ }^{3} P\right)$. 
of particular interest because one spectral scan was obtained as the Earth's atmosphere occluded the Sun, thereby eliminating the EUV background and allowing a more accurate measurement of the X-ray continuum below $10 \AA$. We have selected the resonance lines of O viI, O viII, NeIx, Nex, Mgxi, and Fexvil for study, and the observed fluxes, based on the average of eight spectral scans, are given in Table VIII.

The technique used by Pottasch in his analysis is based on the fact that the ions whose lines he used are each abundant over a narrow temperature range $(\Delta T)$ so that the emission functions of the lines are sharply peaked at a particular temperature $\left(T_{m}\right)$. Emission measure at $T_{m}$ is computed by defining an average emission function over the range $\Delta T$ about $T_{m}$. This technique effectively inverts the line emission integral by the use of lines that have values of $T_{m}$ spread over the temperature interval of interest. This technique cannot be used for the lines of hydrogen- and helium-like ions, which are excited over a broad range of temperatures. We shall use a method of analysis which is similar to the technique used by Chambre (1971). The procedure used is to select a coronal model with several parameters and compute the theoretical line flux by integrating the excitation function, given by Equations (2) or (3), over the assumed coronal temperature structure. The values for the parameters are then selected by minimizing the goodness of fit parameter chi-squared $\left(\chi^{2}\right)$. If we call the emission measure, $n_{\mathrm{e}}^{2} \Delta V$, which is, of course a function of temperature, $S(T)$, the atmosphere structure which we selected is given by

$$
\begin{array}{r}
S(T)=C 10^{-T_{2} / T_{1}}\left[1+1.1515\left(T_{2}-T_{0}\right) / T_{1}-1.1515 B\left(T-T_{0}\right)^{2} / T_{1}\left(T_{2}-T_{0}\right)\right] \\
1 \times 10^{6} \mathrm{~K}<T<T_{0} \\
S(T)=C 10^{-T_{2} / T_{1}}\left[1+1.1515\left(T_{2}-T_{0}\right) / T_{1}-1.1515\left(T-T_{0}\right)^{2} / T_{1}\left(T_{2}-T_{0}\right)\right] \\
T_{0}<T<T_{2} \\
S(T)=C 10^{-T / T_{1}} \\
T>T_{2},
\end{array}
$$

where the parameter $B$ is selected to make $S\left(1 \times 10^{6}\right) / S\left(T_{0}\right)=0.4$. The temperature $T_{0}$ is the most probable coronal temperature, and the condition on $B$ is selected to make the function $S(T)$ agree with the model of Pottasch below the most probable temperature $T_{0}$. The flux in an emission line is then

$$
F_{\text {theor }}=\int \mathrm{d} T S(T)\left[\frac{\mathrm{d}^{2} \phi_{\mathrm{ex}}}{\mathrm{d} t \mathrm{~d} V}+\frac{\mathrm{d}^{2} \phi_{\mathbf{D}}}{\mathrm{d} t \mathrm{~d} V}\right] .
$$

The function $\chi^{2}$ is defined as

$$
\left.\sum_{\text {all lines }}\left[\left(F_{\text {theor }}-F_{\text {exp }}\right)^{2} / D_{\text {exp }}^{2}\right)\right] \text {, }
$$

where $D_{\text {exp }}$ is the experimental error. Values of $T_{0}$ and $T_{2}$ are selected, and $\chi^{2}$ is calculated as a function of $T_{1}$. The $\chi^{2}$ routine automatically calculates the value of $C$. The FeXVII resonance line was not included in this procedure and was later used to determine the iron abundance by normalizing the iron flux computed with the model found 
to that observed. The relative neon and magnesium abundances are rather well restricted because the shape of the Nex and MgXI excitation functions is almost identical. We used three values of the oxygen abundance in the model calculations. The best results were found for a most probable coronal temperature of $2 \times 10^{6} \mathrm{~K}$, in good agreement with the result of previous models.

The minimum value of $\chi^{2}$ was found to occur for $T_{1} \sim 4.0 \times 10^{6} \mathrm{~K}$, and the resultant coronal structure and the values of iron abundance and the values of $\chi^{2}$ found in each case are shown by curves I, II, and III in Figure 25. A fourth model, with a cutoff introduced at $6 \times 10^{6} \mathrm{~K}$ was found to give an equally satisfactory fit provided that $T_{1}$ was set equal to $7.0 \times 10^{6} \mathrm{~K}$.

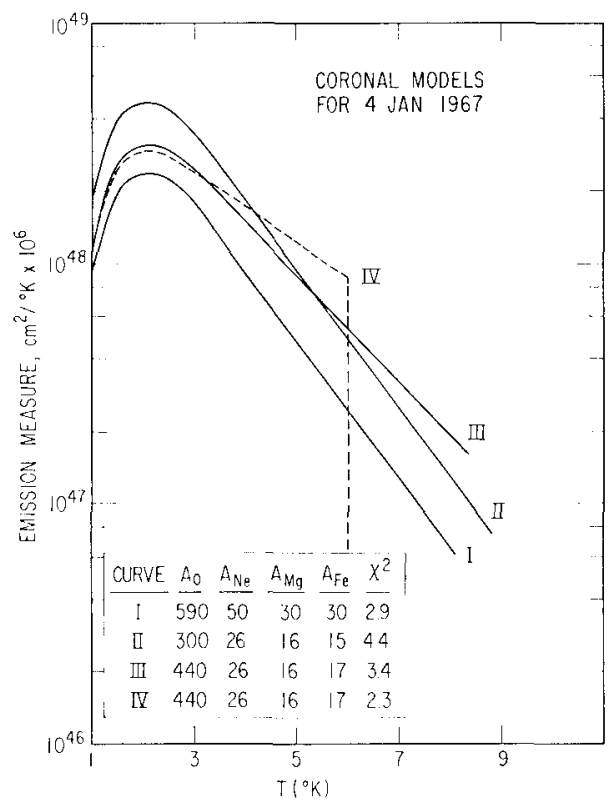

Fig. 25. Coronal models based on the OVI-10 spectrum shown in Figure 2. The model parameters $T_{0}, T_{3}$, and $T_{2}$ (see Equation (8) are I and II, 2, 3.5, $3 \times 10^{6} \mathrm{~K} ; \mathrm{III}, 2,4.5,3 \times 10^{6} \mathrm{~K}$; and IV, 2, 7, $2.5 \times 10^{6} \mathrm{~K}$. The $\mathrm{Fe}$ abundances found, as well as the other abundances used, are shown in the figure.

In order to distinguish between the models with different relative oxygen abundances, and to normalize the abundances to hydrogen, the curves of emission measure were used to compute the expected shape of the X-ray continuum, including the flux due to thermal bremsstrahlung, radiative recombination, and two-photon decay, as given by Equations (5), (6), and (7). The results are compared with the experimentally determined continuum flux in Figure 26. While the agreement is not perfect, this comparison favors those models with a lower oxygen abundance.

\subsection{TEMPERATURE STRUCTURE}

Widing (1966) and Widing and Sandlin (1968) have used the technique developed by 
Pottasch (1967) to analyze the spectral observations made between 30 and $100 \AA$ by Austin et al. (1966) and by Manson (1967), and have derived a coronal model which is in general agreement with the model developed by Pottasch. The model derived by Widing and Sandlin places the most probable temperature in the corona at 1.7-1.8 $\times$ $10^{6} \mathrm{~K}$, in agreement with the earlier analysis of lines in the same spectral region (Austin et al., 1966; Widing, 1966), but at a somewhat higher temperature than the $1.5 \times 10^{6} \mathrm{~K}$ found by Pottasch.

Models based on observations of the spectrum below $25 \AA$ have been strongly influenced by the presence of active regions. Evans and Pounds (1968) found that a three component model, with a disk component at $1.5 \times 10^{6} \mathrm{~K}$ and active region components at 3 and $4 \times 10^{6} \mathrm{~K}$, could explain both the intensities and line profiles of the spectra obtained by Evans et al. (1967) on a Skylark rocket. The line profiles for the lines of O VIII, NeIx, Nex, and Fexvil showed a strong component which was associated with the major active regions on the disk as determined by $9.1 \mathrm{~cm}$ radioheliographs, and a weaker component associated with the sum of the weaker active regions and the whole disk.

The spectral observations made by Batstone et al. (1970) on August 8, 1967, which extended from 5-15 $\AA$, resolved three distinct active regions (see Figure 4) which domiinated the emission from the lines of NeIX, Nex, MgXI, MgXII, and SixIII. A least squares technique was used to fit a four component model, extending from $1.5 \times 10^{6} \mathrm{~K}-$ $9.5 \times 10^{6} \mathrm{~K}$ in $2 \times 10^{6} \mathrm{~K}$ intervals, to the observed line fluxes. These active region models display a strong high energy tail which does not appear to be required for the model, based on the January 4, 1967 fluxes, shown in Figure 25. The active region component present on August 8, was clearly stronger that that present on January 4, since for the August 8 observations the MgIX line intensities are almost as strong as the NeIX line intensities, in contrast to the relative intensities for these lines given in Table VIII for January 4.

Beigman and Vainshtein (1970) have used line fluxes below $25 \AA$ reported by several groups to construct coronal models. Their model is characterized by a sharp cutoff of coronal material at $4 \times 10^{6} \mathrm{~K}$, and the use of a rather high value for the iron abundance $\left(A_{\mathrm{Fe}}=220 \times 10^{-6}\right)$. However, Beigman and Vainshtein point out that the use of a higher cutoff temperature would reduce the iron abundance to a value of $A_{\mathrm{Fe}} \sim$ $60 \times 10^{-6}$.

A comprehensive study of coronal structure has been reported recently by Chambre (1971). Observations of Fexv and Fexvi lines at $\sim 300 \AA$, of the Sivul to Sixil lines at $\sim 40$ to $70 \AA$, and of the O VII, O vIII, FexvII and FexvIII lines between 10 and $25 \AA$ were included. These observations were taken over an extended period of time, and microwave data were used to select a set of spectral observations corresponding to similar solar conditions. Chambre presents a summary of the absolute intensity measurements for the lines which he uses in his analysis. The models derived by Chambre are shown in Figures 27 and 28. The distribution $f_{2}$ shown in Figure 28 indicate the effect of including earlier measurements obtained closer to solar maximum. Figure 28 also compares a number of models, including those of Evans and Pounds (E.P.), of 


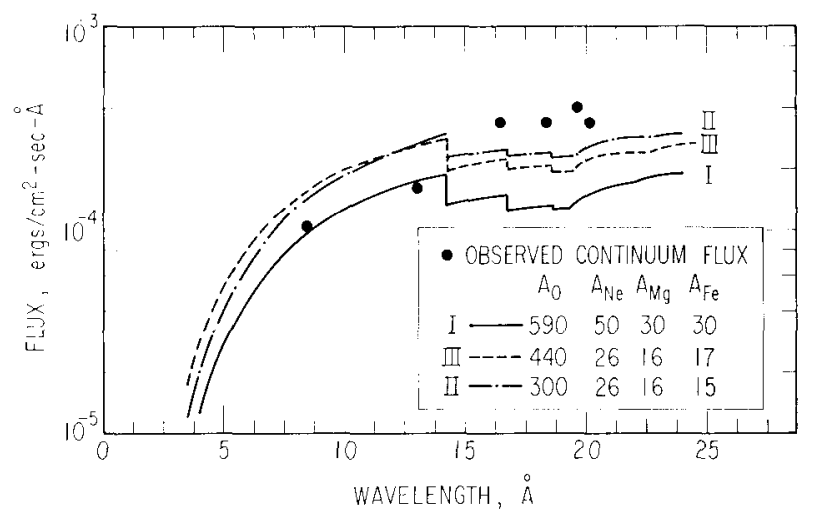

Fig. 26. The X-ray continuum flux observed for the OV1-10 spectrum shown in Figure 2. The theoretical curves shown are computed for the models shown in Figure 25.

Pottasch (P.), and of Broadman and Billings (BB). Comparison of Chambre's model with that shown in Figure 25, suggests that the analysis which includes only lines observed below $25 \AA$ underestimates the emission measure below $2-3 \times 10^{6} \mathrm{~K}$. This might explain the difference between the predicted and observed continuum fluxes in Figure 26, since a larger emission measure at temperatures below $1.5 \times 10^{6} \mathrm{~K}$ would increase the continuum flux near $20 \AA$.

Chambre derives a set of coronal abundances which are characterized by smaller oxygen-to-iron-and-silicon abundance ratios than those found by Withbroe (1971) or in the analysis on OV 1-10 results presented in the previous section.

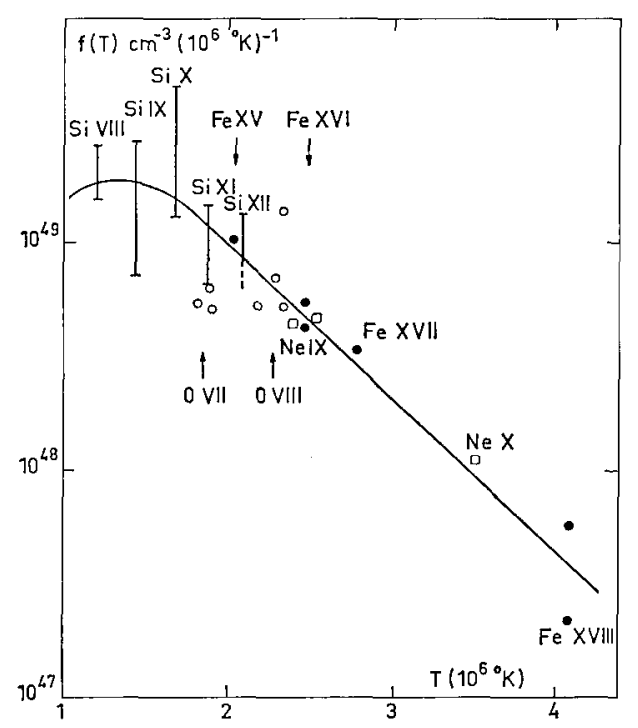

Fig. 27. Coronal model derived by Chambre (1971). 


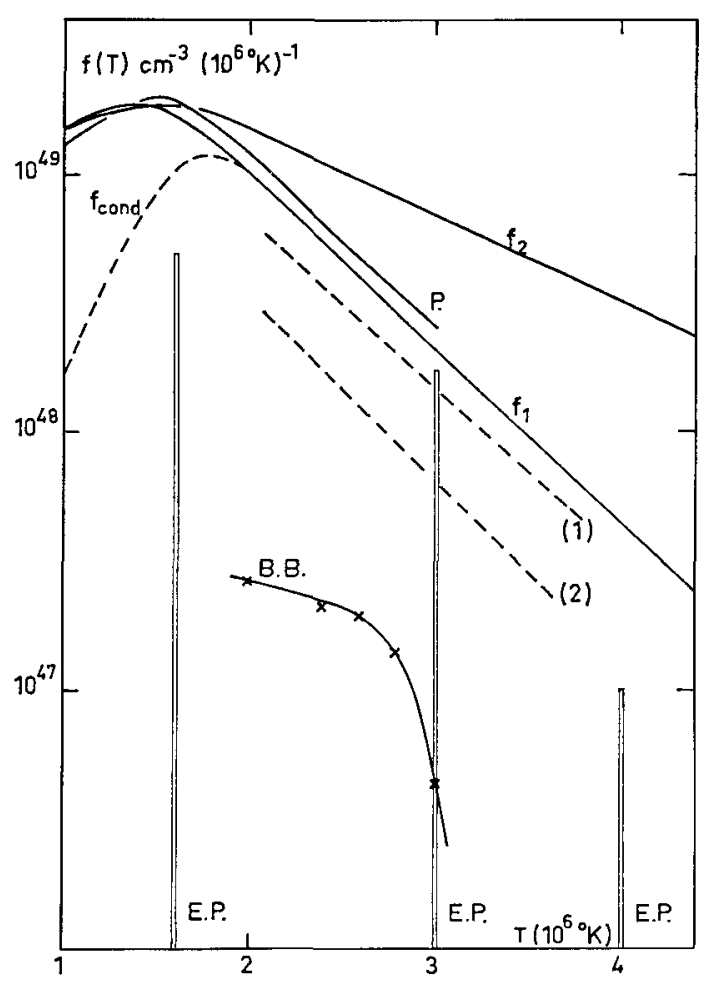

Fig. 28. Comparison of Coronal models derived by Chambre $\left(f_{1}, f_{2}\right)$ Pottasch (P), Evans and Pounds (E. P.) on (1), (2) and by Broadman and Billings (B. B.). The Broadman and Billings model is for a single active region only. The other models are based on whole disk data.

\subsection{Density Structure}

The determination of the density structure of the corona requires high resolution spectroheliograms. Noyes (1971) has discussed the density models which have been constructed with the Harvard OSO-4 and OSO-6 observations. In Section 3.4 several techniques which have been applied to lower spatial resolution spectra were discussed, and preliminary results were presented.

Broadman and Billings (1969) have analyzed the high resolution slitless spectroheliograms obtained by Tousey and Purcell (Tousey, 1967), which have 10" sec resolution. These observations covered the wavelength interval from 170-630 $\AA$, and Broadman and Billings used lines of FeIX, XVI, XIV, NixvIII, MgIX, NeVII, and SixII in their analysis. Their method of analysis is similar to that of Pottasch (1967). However, because of the dependence of emission measure on the square of the electron density, they were able to construct isodensity and isotemperature contours for an active region on the limb based on the intensity distribution in the spectroheliograms. Their model is shown in Figure 29, and includes a high density, high temperature core with rms electron densities of $2 \times 10^{-9} \mathrm{~cm}^{-3}$. The thermal model found for this region by 


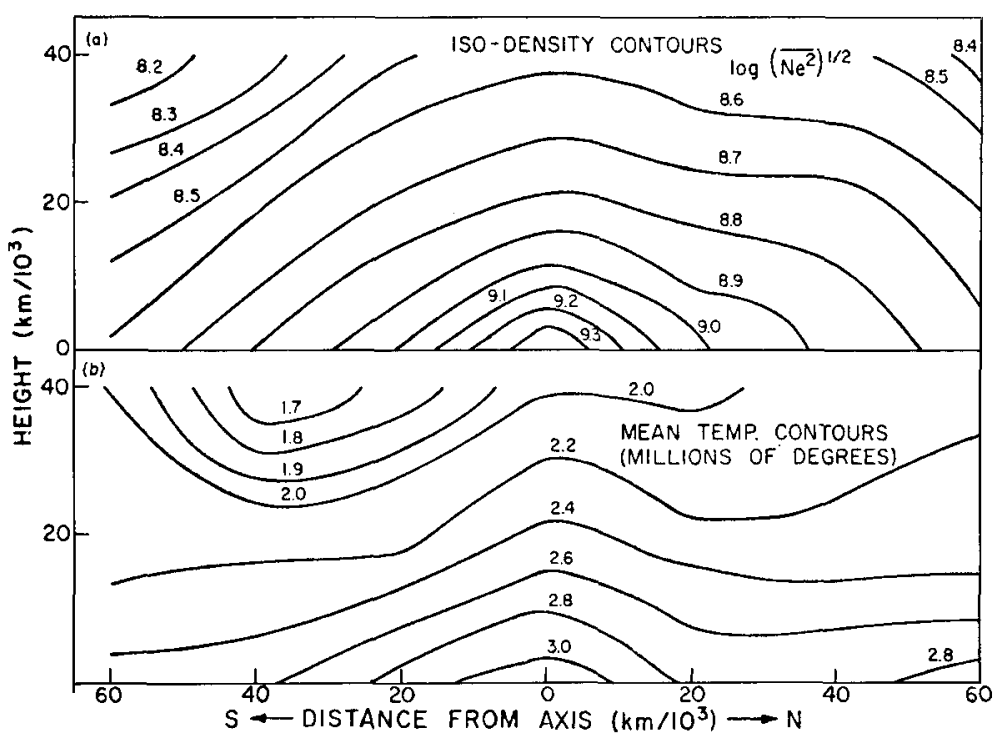

Fig. 29. (a) An rms/density contour map of the limb enhancement of April 28, 1966. (b) The averaged temperatures based on relative emission from EUV lines of different temperatures (Broadman and Billings, 1969).

Broadman and Billings is shown in Figure 28 (curve BB). These authors do not discuss the coronal abundances which they used in their analysis, and the absolute flux scale which was used derived by a somewhat indirect method.

\section{Recent Observational Results and Prospects}

The most serious deficiencies in the presently available observations of the coronal $X$-ray spectrum are the lack of spatial resolution, and the uncertainty in absolute and relative intensity calibrations. This latter problem is, of course, further complicated by the variability of the corona, and the difficulty of comparing observations made at different times.

Recently, groups at Lockheed, and at the University of Leicester have combined multigrid mechanical collimators with Bragg crystal spectrometers to obtain in one case an X-ray spectroheliogram of the OVII and NeIX resonance, intercombination and forbidden lines (Acton et al., 1971), and in the second case the spectrum of an active region between 4 and $22.5 \AA$ (Parkinson, 1971). The observations by the Lockheed group have been used to set an upper limit on density in nonflaring coronal features by using the technique of Gabriel and Jordan (1969b). The observations of the Leicester group have resulted in the discovery of new lithium-like satellite lines near the resonance line of the helium-like ions of O viI, NeIX, Mgxi, and SixIII. A number of other groups are planning similar observations with rocket borne spectroheliographs. 
The launch of two solar satellites, OSO-H, and the Apollo Telescope Mount (ATM) on the Skylab mission will provide platforms for high resolution spectroheliographs. The Goddard Space Flight Center experiment on OSO-H will obtain spectroheliograms with $20^{\prime \prime}$ resolution and $1 \AA$ resolution between 170 and $400 \AA$, and by the use of Ross filters, isolate several emission lines with similar spatial resolution below $25 \AA$. The Naval Research Laboratory experiment on OSO-H includes an EUV spectroheliograph.

The Naval Research Laboratory 'A' experiment on ATM will obtain slitless spectroheliograms between 170 and $600 \AA$ with resolution of a few arcseconds. Purcell and Tousey (1969) have flown a preliminary version of this instrument on a rocket in 1969, and have obtained a beautiful sequence of photographs (Tousey, 1971) which show a $2 \mathrm{~N}$ flare in progress, as well as active regions. Tousey (1971) presents a detailed discussion of these high resolution spectroheliographs, and points out that the chromospheric network extends to lines excited at temperatures of $2 \times 10^{5} \mathrm{~K}$, but that it is not observed in the Ne VII line at $465 \AA$, which is excited near $6 \times 10^{5} \mathrm{~K}$. We may think of the true corona beginning at some point between these two temperatures. The Harvard College Observatory experiment on ATM will obtain spectroheliograms in the wavelength range from 300-1300 $\AA$ (which includes a number of coronal lines) with $5^{\prime \prime}$ resolution. These observations will extend the significant advances in our knowledge of the transition region and lower corona made by the HCO, OSO-4 and OSO-6 spectroheliographs, which covered the same spectral range with lower resolution.

The problem of absolute and relative intensity calibration of X-ray observations over a wide spectral range, particularly for satellite spectrometers, is still a most serious obstacle to the construction of detailed coronal models, and should receive more serious consideration from experimenters.

\section{Acknowledgements}

I would like to thank my colleague at Aerospace, Dr H. R. Rugge, for many helpful discussions and for a critical reading of the manuscript. I would also like to thank many others who allowed me to see manuscripts and results in advance of publication and gave permission for these results to be quoted, and in some cases reproduced. I would especially like to thank Drs A. Dalgarno and G. Withbroe of HCO; Drs G. Doschek and R. Tousey of NRL; Dr A. Gabriel of ARU, Culham; J. Manson of AFCRL; Dr W. Neupert of GSFC; and Dr J. Zirker of Hawaii. I would also like to thank Dr H. Hilton, Mrs M. Wray and Mrs M. E. Brennan for invaluable assistance in the coronal spectrum and atmospheric structure model computations, and Mrs C. Roth for assistance with the manuscript.

\section{References}

Acton, L. W., Catura, R. C., Meyerott, H. J., and Culhane, J. L.: 1971, Nature 223, 75.

Allen, C. W.: 1963, Astrophysical Quantities, 2nd ed., Athlone Press, London.

Allen, J. and Dupree, A. K.: 1969, Astrophys. J. 155, 27. 
Argo, H. V., Bergey, J. A., Evans, W. D., and Singer, S.: 1968, Solar Phys. 5, 531.

Austin, W. E., Purcell, J. D., and Tousey, R.: 1962, Astron. J. 67, 110.

Austin, W. E., Purcell, J. D., Tousey, R., and Widing, K. G.: 1966, Astrophys. J. 145, 373.

Austin, W. E., Purcell, J. D., Snider, C. B., Tousey, R., and Widing, K. G.: 1967, in R. L. SmithRose (ed.), Space Res. 7, North-Holland Publ. Co., Amsterdam, p. 1252.

Bame, S. J., Asbridge, J. R., Hurdhausen, A. J., and Montgomery, M. P.: 1970, J. Geophys, Res. 75,6360 .

Batstone, R. M., Evans, K., Parkinson, J. H., and Pounds, K. A.: 1970, Solar Phys. 13, 389.

Bedo, D. E. and Hinteregger, H. E., 1965, Jap. J. Appl. Phys. 4, Suppl. 1, 473.

Behring, W. E., Neupert, W. M., and Lindsay, J. C.: 1963, in W. Priester (ed), Space Res. 3, NorthHolland Publ. Co., Amsterdam, p. 814.

Behring, W. E., Cohen, L., and Feldman, U.: 1971, 'The Solar Spectrum from 60-385 Å', GSFC Preprint.

Beigman, I. L, and Vainshtein, L. A.: 1967a, On the X Radiation of the Solar Corona, Lebedev Inst. Lab. of Spectrosc. Reprint 104.

Beigman, I. L. and Vainshtein, L. A.: 1967b, Astron Zh. 44, 668 (English Transl. 1967, Soviet Astron.-AJ 11, 531).

Beigman, I. L. and Unnov, A. M.: 1970, Soviet J. Opt. Spectrosc. 27, 380 (GSFC Trans. ST-SPC-NP10819).

Beigman, I. L. and Vainshtein, L. A.: 1970, Astron. Zh. 47, 1030 (English. Transl. 1971, Soviet Astron.-AJ 14, 825).

Beigman, I. L., Vainshtein, L. A., and Syunyaev, R. A.: 1968, Usp. Fiz. Nauk 95, 267 (English Transl. 1968, Soviet Phys.-Usp. 11, 411).

Beigman, I. L., Vainshtein, L. A, and Vinogradov, A : 1969, Astron. Zh. 46, 985 (English Transl. 1970, Soviet Astron.-AJ 13,775).

Beigman, I. L., Vainshtein, L. A., and Urnov, A. M.: 1971, Thermal Radiation of the Sun Below $30 \AA$, Lebedev Inst. Lab. of Spectrosc. Reprint 28.

Bely, O.: 1966a, Proc. Phys. Soc. 88, 587.

Bely, O.: 1966b, Ann. Astrophys. 29, 131.

Bely, O.: 1967, Ann. Astrophys. 30, 953.

Bely, O. and Bely, F.: 1967, Solar Phys. 2, 285.

Bely, O. and Blaha, M.: 1968, Solar Phys. 3, 563.

Bely, O. and Fraucher, P.: 1969, Astron. Astrophys. 1, 37.

Bely, O. and Petrini, D.: 1970, Astron. Astrophys. 6, 318.

Bely, O. and Van Regemorter, H.: 1970, Ann. Rev. Astron. Astrophys. 8, Ann. Rev. Inc., Palo Alto, p. 329.

Blake, R. L. and House, L. L.: 1971, Astrophys, J. 166, 423.

Blake, R. L., Chubb, T. A., Friedman, H., and Unzicker, A. E.: 1964, Science 146, 1037.

Blake, R. L., Chubb, T. A., Friedman, H., and Unzicker, A. E.: 1965a, Ann. Astrophys. $28,583$.

Blake, R. L., Chubb, T. A., Friedman, H., and Unzicker, A. E.: 1965b, Astrophys. J. $142,1$.

Blumenthal, G. R., Drake, G. W. F., and Tucker, W. H.: 1972, Astrophys. J. 172, 205.

Broadman, W. J. and Billings, D. E.: 1969, Astrophys. J. 156, 731.

Burgess, A.: 1964, Astrophys, J. 139, 776.

Burgess, A. and Summers, H. P.: 1969, Astrophys. J. 157, 1007.

Burgess, A., Hummer, D. G., and Tully, J. A.: 1970, Phil. Trans. Roy. Soy. London A266, 225.

Burton, W. M. and Wilson, R.: 1965, Nature 267, 61.

Chambre, G.: 1971, Astron. Astrophys. 12, 210.

Chapman, R. D.: 1969, Astrophys. J. 156, 87.

Cohen, L., Feldman, U., and Kastner, S. O.: 1968, J. Opt. Soc. Amer. 58, 331.

Cohen, L., Feldman, U., and Kastner, S. O.: 1971, J. Opt. Soc. Amer. 61, 91.

Cohen, L., Feldman, U., Swartz, M., and Underwood, J. H.: 1968, J. Opt. Soc. Amer. 58, 843.

Connerade, J. P.: 1971, Astrophys. J. 163, 417.

Connerade, J. P., Peacock, N. J., and Speer, R. J.: 1970, Solar Phys. 14, 159.

Connerade, J. P., Peacock, N. J., and Speer, R. J.: 1971, Solar Phys. 18,63.

Cooper, J. W., Connelly, M. J., Smith, K., and Ormonde, S.: 1970, Phys. Rev. Letters $25,1540$.

Culhane, J. L.: 1969, Monthly Notices Roy. Astron. Soc. 144, 375. 
Dalgano, A.: 1969, in F. Bopp and H. Kleinpopper (eds.), Physics of One- and Two-Electron Atoms, North-Holland Publ. Co., Amsterdam, p. 261.

Dalgano, A.: 1971, 'Radiative Transitions in the Helium Isoelectronic Sequence', presentation at the Donald H. Menzel Symp., Harvard College Obs., Cambridge, Mass. (NBS Special Pub. No 353).

Dalgano, A. and Drake, G. W. F.: 1969, Les Transitions Interdites dans Les Spectres des Astres, Les Congrès et Colloques de L'Université de Liège, Vol. 54, Univ. of Liege, Belgium, p. 69.

Doschek, G. A. and Meekins, J. F.: 1970, 'Low Intensity Solar X-Ray Flar Spectra From OSO 4', presentation Amer. Geophys. Union, Washington, D.C.

Doschek, G. A. and Meekins, J. F.: 1971, Solar Phys. 13, 220.

Doschek, G. A., Meekins, J. F., Kreplin, R. W., and Chubb, T. A.: 1971a, 'An Analysis of the Soft X-Ray Emission Line Behavior During Solar Flares', presentation XIV COSPAR, Seattle.

Doschek, G. A., Meekins, J. F., Kreplin, R. W., Chubb, T. A., and Friedman, H., 1971b, Astrophys. $J .170,573$.

Doschek, G. A., Meekins, J. F., Kreplin, R. W., Chubb, T. A., and Friedman, H.: 1971c, Astrophys. J. 164, 165.

Drake, G. W. F.: 1969, Astrophys. J. 158, 1199.

Drake, G. W. F.: 1971a, Phys. Rev. A3, 908.

Drake, G. W. F.: 1971b, Astrophys. J. 163, 439.

Drake, G. W. F., Victor, G. A., and Dalgano, A.: 1969, Phys. Rev. 180, 25.

Dupree, A. K.: 1968, Astrophys. Letters 1, 125.

Dupree, A. K. and Reeves, E. M.: 1971, Astrophys. J. 165, 599.

Ederer, D. L., Lucatorto, T., and Madden, R. P.: 1970, Phys. Rev. Letters 25, 1537.

Elton, R. C., Palumbo, L. J., and Griem, H. R.: 1968, Phys. Rev. Letters 20, 783.

Evans, K. and Pounds, K. A.: 1968, Astrophys. J. 152, 319.

Evans, K., Pounds, K. A., and Culhane, L.: 1967, Nature 214, 41.

Evans, D. W., Argo, H. V., Bergey, J. A., Henke, B. L., and Montgomery, M. D.: 1966, Abstracts of Conf. on UV and X-Ray Spectrosc. of Laboratory and Astrophysical Plasmas, Culham, Abingdon, Birks.

Fawcett, B. C.: 1970, J. Phys. B 3, 1152.

Fawcett, B. C.: 1971a, Wavelengths and Classifications of Emission Lines due to $2 s^{2} 2 p^{n}-2 s 2 p^{n+1}$ and $2 s 2 p^{\mathrm{n}+1}$ Transitions, ARU Report R2, Culham Laboratory, Abingdon, Birkshire.

Fawcett, B. C.: 1971b, J. Phys. B 4, 981.

Fawcett, B. C., Gabriel, A. H., and Saunders, P. A. H.: 1967, Proc. Phys. Soc. $90,963$.

Fawcett, B. C., Peacock, N. J., and Cowan, R. D.: 1968, J. Phys. B 1, 295.

Fawcett, B. C., Hardcastle, R. A., and Tondello, G.: 1970, J. Phys. B 3, 564.

Fawcett, B. C., Gabriel, A. H., and Paget, T. M.: 1971, J. Phys. B 4, 986.

Feinberg, G. and Sucher, J.: 1971, Phys. Rev. Letters 26, 681.

Feldman, U. and Cohen, L.: 1968, Astrophys. J. 151, L55.

Feldman, U. and Cohen, L.: 1970, Astrophys. J. L105.

Feldman, U., Behring, W., and Cohen, L.: 1972, 'Wavelengths of Solar Lines in the 380-50 Å Region and Their Identification,' presentation LAU Coll. No. 14, Utrecht.

Feldman, U., Cohen, L., and Behring, W.: 1970, J. Opt. Soc. Amer. 60, 891.

Feldman, U., Katz, L., Behring, W., and Cohen, L.: 1971, J. Opt. Soc. Amer. 61, 91.

Flower, D. R.: 1972, Astron. Astrophys. 17, 201.

Freeman, F. F. and Jones, B. B.: 1970, Solar Phys. 15, 298.

Freeman, F. F., Gabriel, A. H., Jones, B. B., and Jordan, Carole: 1971, Phil. Trans. Roy. Soc. London A270. 127.

Fritz, G., Kreplin, R. W., Meekins, J. F., Unzicker, A. E., and Friedman, H.: 1967, Astrophys. J. 148, L133.

Gabriel, A. H.: 1970, in C. de Jager (ed.), Highlights of Astronomy, XIV IAU General Assembly, Brighton, Vol. 2, D. Reidel Publ. Co., Dordrecht, Holland, p. 486.

Gabriel, A. H.: 1972, this issue, p. 655.

Gabriel, A. H.: 1972, 'Dielectronic Satellite Spectra for Highly-Charged Helium-Like Ion Lines', to be published in Monthly Notices Roy. Astron. Soc.

Gabriel, A. H. and Jordan, Carole: 1969a, Nature 221, 947.

Gabriel, A. H. and Jordan, Carole: 1969b, Monthly Notices Roy. Astron. Soc. 145, 241.

Gabriel, A. H. and Jordan, Carole: 1970, Phys. Letters 32A, 166. 
Gabriel, A. H. and Paget, T. M.: 1972, J. Phys. B. 5, 673.

Gabriel, A. H., Fawcett, B. C., and Jordan, Carole: 1966, Proc. Phys. Soc. 87, 825.

Garstang, R. H.: 1967, Publ. Astron. Soc. Pacific 78, 399.

Garstang, R. H.: 1969a, Publ. Astron. Soc. Pacific 81, 488.

Garstang, R. H.: 1969b, Les Transitions Interdites dans Les Spectres des Astres, Congrès et Colloques de L'Université de Liège, Vol. 54, Univ. of Liege, Belgium, p. 35.

Garstang, R. H.: 1969c, 'Forbidden Atomic Transitions', presentation NATO Summer Inst. on New Directions in Atomic Physics, Ismir, Turkey.

Gartz, T., Kock, M., Richter, J., Boschek, B., Hotweger, H., and Unsold, A.: 1969a, Nature 222, 1254.

Gartz, T., Holweger, H., Kock, M., and Richter, J.: 1969b, Astron. Astrophys. 2, 446.

Goldberg, L.: 1967, Ann. Rev. Astron. Astrophys. 5, Ann. Rev. Inc., Palo Alto, p. 279.

Goldsmith, S., Feldman, U., and Cohen, L.: 1971, J. Opt. Soc. Amer. 61, 615.

Griem, H.: 1969, Astrophys. J. 156, L103. (See also 1970, Astrophys. J. 161, L155.)

Grineva, Y. I., Karev, V. I., Korneev, V. V., Krutov, V. V., Mandel'stam, S. L., Vainshtein, L. A., Vaisiljev, B. N., and Zitnik, I. A.: 1971, 'A Study of the Solar X-ray Emission from the Satellite Intercosmos 4 and the Rocket Vertical-1', presentation XIV COSPAR, Seattle; to be published in Kosmich. Issled.

Hall, L. A. and Hinteregger, H. E.: 1970, J. Geophys. Res. 75, 6959.

Hall, L. A., Schweizer, W., Heroux, L., and Hinteregger, H. E.: 1965a, Astrophys. J. 142, 13.

Hall, L. A., Schweizer, W., and Hinteregger, H. E.: 1965b, J. Geophys. Res. 70, 2241.

Hall, L. A., Higgins, J. E., Chagon, C. W., and Hinteregger, H. E.: 1969, J.Geophys. Res. 74, 4181. Heroux, L.: 1964, Proc. Phys. Soc. 83, 121.

Herring, J. R. H., Glencross, W. N., Parkinson, J. H., and Pounds, K. A.: 1971, Proc. Roy. Soc. London A321, 493.

Hinteregger, H. E.: 1965, in C. de Jager (ed.), The Solar Spectrum, D. Reidel Publ. Co., Dordrecht, p. 179.

Hinteregger, H. E.: 1970, Ann. Geophys. 26, 547.

Hinteregger, H. E. and Hall, L. A.: 1969, Solar Phys. 6, 175.

Hinteregger, H. E., Hall, L. A., and Schweizer, W.: 1964, Astrophys. J. 140, 319.

Holtzer, T. E. and Axford, W. J., 1970, J. Geophys. Res. 75, 6354.

Hoory, S., Feldman, U., Goldsmith, S., Behring, W., and Cohen, L.: 1970, J. Opt. Soc. Amer. 60, 1449.

Hoory, S., Goldsmith, S., Feldman, U., Behring, W., and Cohen, L.: 1971, J. Opt. Soc. Amer. 61, 504.

Ivanov-Kholodny, G. S. and Nikolsky, G. M.: 1963, in W. Priester (ed.), Space Res. 3, North-Holland Publ. Co., Amsterdam, p. 787.

Jacob, A.: 1968, Solar Phys. 5, 359.

Johnson, W. D. and Kunze, H. J.: 1971, Phys, Rev. A4, 25.

Jones, B. B., Freeman, F. F., and Wilson, R.: 1968, Nature 219, 252.

Jordan, Carole: 1965, Comm. Univ. London Obs. No. 68.

Jordan, Carole: 1966, Monthly Notices Roy. Astron. Soc. 132, 515.

Jordan, Carole: 1969, Monthly Notices Roy. Astron. Soc. 142, 499.

Jordan, Carole: 1970, Monthly Notices Roy. Astron. Soc. 149, 1.

Jordan, Carole: 1971, in C. de Jager (ed.), Highlights of Astronomy, XIV IAU General Assembly, Brighton, Vol. 2, D. Reidel Publ. Co., Dordrecht, Holland, p. 519.

Jordan, Carole and Pottasch, S. R.: 1968, Solar Phys. 4, 104.

Karzas, W. and Latter, R.: 1961, Astrophys. J. Suppl. 6, 167.

Kelly, R. L.: 1968, Atomic Emission Lines Below 2000 Ángströms, NRL Report 6648.

Krieger, A., Paolini, F., Vaiana, G. S., and Webb, D.: 1972, Solar Phys. 22, 150.

Lambert, D. L.: 1967, Nature 215, 44.

Landini, M. and Monsignori Fossi, B. C.: 1970, Astron. Astrophys. 6, 468.

Landini, M. and Monsignori Fossi, B. C.: 1972, this issue, p. 586.

Lange, J. and Scherb, F.: 1970, J. Geophys. Res. 75, 6350.

Lazer, D. and Garstang, R. H.: 1968, Ann. Rev. Astron. Astrophys. 6, Ann. Rev. Inc., Palo Alto, p. 449.

Lie, T. N. and Elton, R. C.: 1971, Phys. Rev. A3, 965. 
Mandel'stam, S. L.: 1967, Appl. Opt. 6, 1934.

Manson, J.: 1967, Astrophys. J. 147, 703.

Manson, J.: 1968, Astrophys. J. 153, L191.

Manson, J.: 1972, 'Measurements of the Solar Spectrum Between 30 and $128 \AA$ ', AFCRL Preprint.

Marrus, R. and Schmieder, R. W.: 1970a, Phys. Rev. Letters 25, 1245.

Marrus, R. and Schmieder, R. W.: 1970b, Phys. Rev. Letters p. 1689.

McWhirter, R. W. P. and Hearn, A. G.: 1963, Proc. Phys. Soc. 82, 641.

Meekins, J. F., Kreplin, R. W., Chubb, T. A., and Friedman, H.: 1968, Science 162, 891.

Meekins, J. F., Doschek, G. A., Friedman, H., Chubb, T. A., and Kreplin, R. W.: 1970, Solar Phys. 13, 198.

Mewe, R.: 1971a, this issue, p. 666.

Mewe, R.: 1972b, Solar Phys. 22, 459.

Moore, C. E.: 1949, Atomic Energy Levels, Vol. 1, NBS Circular 467.

Moore, C. E.: 1952, Atomic Energy Levels, Vol. II.

Munro, R. H., Dupree, A. K., and Withbroe, G. L.: 1971, Solar Phys. 19, 347.

Neupert, W. N.: 1967, Solar Phys. 2, 294.

Neupert, W. N.: 1969, Ann. Rev. Astron. Astrophys. 7, Ann. Rev. Inc., Palo Alto, p. 1121.

Neupert, W. N.: 1971a, in C.J. Macris (ed.), Physics of the Solar Corona, D. Reidel Publ. Co.,

Dordrecht, p. 237.

Neupert, W. N.: 1971b, Solar Phys. $18,474$.

Neupert, W. M. and Behring, W. E.: 1962, J. Quant. Spectr. Radiative Transfer 2, 527.

Neupert, W. M. and Swartz, M.: 1970, Astrophys. J. 160, L189.

Neupert, W. M., Behring, W. E., and Lindsay, J. C.: 1964, in P. Muller (ed.), Space Res. 4, NorthHolland Publ. Co., Amsterdam, p. 719.

Neupert, W. M., Gates, W., Swartz, M., and Young, R.: 1967, Astrophys. J. 149, L79.

Noyes, R. W.: 1971, Ann. Rev. Astron. Astrophys. 9, Ann. Rev. Inc., Palo Alto, p. 209.

Osterbrock, D. E.: 1970a, J. Phys. B 3, 149.

Osterbrock, D. E.: 1970b, Astrophys. J. 160, 25.

Parkinson, J. H.: 1971, Nature 233, 44.

Parkinson, J. H. and Pounds, K. A.: 1971, Solar Phys. 17, 146.

Peacock, N. J., Speer, R. J., and Hobby, M. G.: 1969, J. Phys. B 2, 799.

Petrini, D.: 1969, Astron. Astrophys. 1, 139.

Pottasch, S.: 1963, Astrophys. J. 137, 945.

Pottasch, S.: 1964, Space Sci. Rev. 3, 816.

Pottasch, S. : 1966, Bull. Astron. Inst. Neth. 18, 237.

Pottasch, S. : 1967, Bull. Astron. Inst. Neth. 19, 113.

Pounds, K. A.: 1970, Ann. Geophys. 26, 555.

Purcell, J. D. and Tousey, R.: 1969, Bull. Amer. Astron. Soc. 1, 290.

Reeves, E. M. and Parkinson, W. H.: 1970, Astrophys. J. Suppl. 181, 1.

Robbins, D. E., Hundhausen, A. J., and Bame, S. J.: 1970, J. Geophys. Res. 75, 1178.

Roth, N. V. and Elton, R. C.: 1968, Measurement and Identification of Laboratory-Produced Vacuum Ultraviolet Spectral Lines, NRL Report 6638.

Rugge, H. R.: 1969 , private communication.

Rugge, H. R. and Walker, A. B. C.: 1968, in A. P. Mitra, L. G. Jacchia, and W. S. Newman (eds.), Space Res. 8, North-Holland Publ. Co., Amsterdam, p. 439.

Rugge, H. R. and Walker, A. B. C.: 1971a, Solar Phys. 15, 372.

Rugge, H. R. and Walker, A. B. C.: 1971b, Solar Phys. 18, 244.

Rugge, H. R., Walker, A. B. C., and Anderson, M.: 1970, Correlations Between Solar X-Ray Line Emission and Solar Radio Emission, The Aerospace Corp. Report TR-0059(9260-02)-1.

Schmieder, K. W. and Marrus, K.: 1970, Phys. Rev. Letters 25, 1692.

Schweizer, W. and Schmidtke, G.: 1971, Astrophys. J. 169, L27.

Seaton, M. J.: 1969, in B. Bederson, V. W. Cohen, and F. M. J. Pechanch (eds.), Atomic Physics, Plenum Press, New York, p. 295.

Shore, B.: 1969, Astrophys. J. 158, 1205.

Shore, B. and Mentzel, D. H.: 1968, Principles of Atomic Physics, John Wiley and Sons, Inc., New York.

Smith, M. W. and Wiese, W. L.: 1971, Astrophys. J. Suppl. 23, 103. 
Smith, T. S.: 1969, Les Transitions Interdites dans Les Spectres des Astres, Les Congrès et Colloques de L'Université de Liège, Vol. 54, Univ. of Liège, Belgium, p. 243.

Space Science Board, 1969: United States Space Program Report to COSPAR XII, Nat. Acad. Sci.Nat. Res. Council, Washington, D. C., p. 29.

Space Science Board, 1969: United States Space Science Program Report to COSPAR XIII, Nat. Acad. Sci.-Nat. Res. Council, Washington, D. C., p. 28.

Space Science Board, 1969: United States Spaces Science Program Report to COSPAR XIV, Nat. Acad. Sci.-Nat. Res. Council, Washington, D. C., p. 75.

Spitzer, L. and Greenstein, J. L.: 1951, Astrophys. J. 114, 407.

Tondello, G.: 1969, J. Phys. B 2, 727.

Tousey, R.: 1967, Astrophys. J. 149, 239.

Tousey, R.: 1971, Proc. Phil. Soc. London A270, 59.

Tousey, R., Austin, W. E., Purcell, J. D., and Widing, K. G.: 1963, Space Res. North-Holland Publ Co., Amsterdam, p. 772.

Tousey, R., Austin, W. E., Prucell, J. D., and Widing, K. G.: 1965, Ann. Astrophys. 28, 755.

Tousey, R., Purcell, J. D., Austin, W. E., Garrett, D. L., and Widing, K. G.: 1964, in P. Muller (ed.), Space Res. 4, North-Holland Publ. Co., Amsterdam, p. 703.

Trefftz, E.: 1967, Z. Astrophys. 65, 299.

Trefftz, E.: 1969, in F. Bopp and H. Kleinpopper (eds.), Physics of One- and Two-Electron Atoms, North-Holland Publ. Co., Amsterdam, p. 839.

Trefftz, E.: 1970, J. Phys. B 3, 763.

Tucker, W. H. and Koran, M.: 1971, Astrophys. J. 168, 283.

Underwood, J. H.: 1968, Science 159, 383.

Underwood, J. H. and Muney, W. S.: 1967, Solar Phys. 1, 129.

Vaiana, G. S., Reidy, W. P., Zehnpfennig, T., Van Speybrook, L., and Giacconi, R.: 1968, Science 161, 564.

Vainshtein, L. A. and Sefronova, U. I.: 1971, Astron. Zh. 47, 223 (English Transl. 1971, Soviet Astron.-AJ 15, 175).

Van Regemorter, H.: 1962, Astrophys. J. 136, 906.

Violett, T. and Rense, W. A.: 1953, Astrophys. J. 130, 954.

Walker, A. B. C. and Rugge, H. R.: 1968, Astron. J. 73, 581.

Walker, A. B. C. and Rugge, H. R.: 1969, in C. de Jager and Z. Švestka (eds.), Solar Flares and Space Research, North-Holland Publ. Co., Amsterdam,p. 102.

Walker, A. B. C. and Rugge, H. R.: 1970, Astron. Astrophys. 5, 4.

Walker, A. B. C. and Rugge, H. R.: 1971a, unpublished OVI-10 observations.

Walker, A. B. C. and Rugge, H. R.: 1971b, Astrophys. J. 164, 181

Walker, A. B. C. and Rugge, H. R.: 1971c, unpublished OVI-17 observations.

Widing, K. G.: 1966, Astrophys. J. 145, 380.

Widing, K. G. and Porter, J. R.: 1965, Ann. Astrophys. 28, 779.

Widing, K. G. and Sandlin, G. D.: 1968, Astrophys. J. 152, 545.

Wiese, W. L., Smith, M. W., and Lenonn, G.: 1966, Atomic Transition Probabilities, Vol. 1, NBRDSNBS-4, U.S. Govt. Printing Office, Washington, D.C.

Wiese, W. L., Smith, W. M., and Miles, B. M.: 1969, Atomic Transition Probabilities, Vol. II, NBRDS-NBS-22, U.S. Grovt. Printing Office, Washington, D.C.

Withbroe, G. L.: 1970a, Solar Phys. 11, 42.

Withbroe, G. L.: 1970b, Solar Phys. 11, 208.

Withbroe, G. L.: 1971, The Chemical Composition of the Photosphere and Corona, presentation at the Donald H. Menzel Symp., Harvard College Obs., Cambridge, Mass. (NBS Special Publ. No. 353).

Wooley, R. V. D. R. and Allen, C. W. Z.: 1948, Monthly Notices Roy. Astron. Soc. 108, 292.

Zhitnik, I. A., Krutov, V. V., Maljavkin, L. P., Mandel'stam, S. L., and Cheremukhin, G. S.: 1965, Kosmich. Issled. 5, 276.

Zhitnik, I. A., Krutov, V. V., Maljavkin, L. P., Mandel'stam, S. L., and Cheremukhin, G. S.: 1967, in R. L. Smith-Rose (ed.), Space Res. 7, North-Holland Publ. Co., Amsterdam, p. 1263.

Zirker, J.: 1971, 'The Corona', presentation at the Donald H. Menzel Symp., Harvard College Obs., Cambridge, Mass. (NBS Special Publ. No. 353).

Zirin, H.: 1964, Astrophys. J. 140, 1332. 
Zirin, H., Hall, L. A., and Hinteregger, H. E.: 1963, in W. Priester (ed.), Space Res. 3, North-Holland Publ. Co., Amsterdam, p. 760.

\section{DISCUSSION}

B. S. Fraenkel: Are the $\mathrm{LiF}$ crystals with homogeneous rocking curves all over $2-3 \mathrm{~cm}$ ?

A. B. C. Walker: None of the spectra I have shown were obtained with LiF crystals. The shortest wavelength spectra were obtained with EDDT. However, we have also observed doubly peaked LiF rocking curves, and one should be careful in selecting such crystals for satellite experiments.

C. de Jager: Have you an explanation for the difference in gradients of the observed and computed continuous spectra in the shortest wavelength range.

A. B. C. Walker: One must first realise that the observation of the X-ray continuum is difficult. The counting rates near $20 \AA$ are low, while corrections must be made for scattered light at wavelengths below $15 \AA$. In the case of the spectra I used to obtain continuum fluxes near $8 \AA$ atmospheric attenuation of EUV radiation, as the earth was about to occlude the satellite, eliminated the need for the substraction of scattered light backgrounds. We have averaged the results of 8 spectral scans to increase the statistical accuracy of the fluxes near $20 \AA$. However, in view of the difficulty of the measurement, I do not believe that the observed disagreement between computed and calculated continuum shapes can be considered significant. 